\title{
IV. A new table of standard wave-lengths
}

\section{Prof. Henry A. Rowland}

To cite this article: Prof. Henry A. Rowland (1893) IV. A new table of standard wave-lengths, Philosophical Magazine Series 5, 36:218, 49-75, DOI: 10.1080/14786449308620449

To link to this article: http://dx.doi.org/10.1080/14786449308620449

$$
\text { 曲 Published online: } 08 \text { May } 2009 .
$$

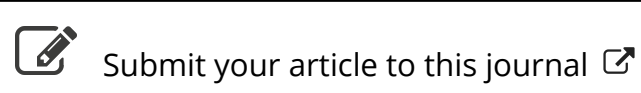

\footnotetext{
Џ Article views: 2
}

Q View related articles ๘

Citing articles: 4 View citing articles $\square$ 


\section{$\left[\begin{array}{ll}49 & \end{array}\right]$ \\ IV. A New Table of Standard Wave-lengths. By Prof. Henry A. Rowland*.}

DURING the last ten years I have made many observations of wave-lengths, and have published a preliminary and a final table of the wave-lengths of several hundred lines in the solar spectrum.

For the purpose of a new table I have worked over all my old observations, besides many thousand new ones, principally made on photographs, and have added measurements of metallic lines so as to make the number of standards nearly one thousand.

Nearly all the new measurements have been made on a new measuring-machine whose screw was specially made by my process $\dagger$ to correspond with the plates and to measure wave-lengths direct with only a small correction.

The new measures were made by Mr. L. E. Jewell, who has now become so expert as to have the probable error of one setting about $\frac{1}{100} \overline{0}$ division of Angström, or 1 part in $5,000,000$ of the wave-length. Many of these observations, however, being made with different measuring-instruments, and before such experience had been obtained, have a greater probable error. This is especially true of those measurements made with eye-observations on the spectrum direct. The reductions of the readings were made by myself.

Many gratings of 6 in. diameter and $21 \frac{1}{2} \mathrm{ft}$. radius were used; and the observations were extended over about ten years.

The standard wave-length was obtained as follows:Dr. Bell's value of $\mathrm{D}_{1}$ was first slightly corrected and became $5896 \cdot 20$. C. S. Peirce's value of the same line was corrected as the result of some measurements made on his grating and became 5896:20. The values of the wave-length then become :-

\begin{tabular}{|c|c|c|}
\hline Weight. & Observer. & D. \\
\hline $\begin{array}{l}1 . \\
2 . \\
2 . \\
5 . \\
10 .\end{array}$ & 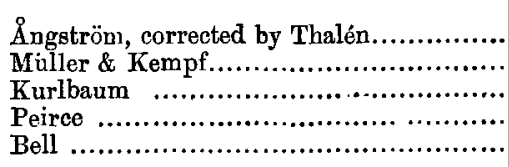 & $\begin{array}{l}5895 \cdot 81 \\
5896 \cdot 25 \\
5895 \cdot 90 \\
5896 \cdot 20 \\
5896 \cdot 20\end{array}$ \\
\hline & Mean............ & $5896 \cdot 156$ \\
\hline
\end{tabular}

* Reprinted from a separate copy from the 'Astronom. and AsteroPhysics" communicated by the Author.

$\dagger$ See Encyc. Brit., art. Screw.

Phil. Mag. S. 5. Vol. 36. No. 218. July 1893. 
As the relative values are more important for spectroscopic work than the absolute, I take this value without further remark. It was utilized as follows :-

1st. By the method of coincidences with the concave grating, the wave-lengths of 14 more lines throughout the visible spectrum were determined from this with great accuracy for primary standards.

2nd. The solar standards were measured from one end of the spectrum to the other many times; and a curve of error drawn to correct to these primary standards.

3rd. Flat gratings were also used.

4th. Measurements of photographic plates from 10 to 19 inches long were made. These plates had upon them two portions of the solar spectrum of different orders. Thus the blue, violet, and ultra-violet spectra were compared with the visible spectrum, giving many checks on the first series of standards.

5th. Measurements were made of photographic plates having the solar spectrum in coincidence with metallic spectra, often of three orders, thus giving the relative wavelengths of three points in the spectrum.

Often the same line in the ultra-violet had its wave-length determined by two different routes back to two different lines of the visible spectrum. The agreement of these to $\frac{1}{100}$ division of Ångström in nearly every case showed the accuracy of the work.

6th. Finally, the important lines had from 10 to 20 measurements on them, connecting them with their neighbours and many points in the spectrum, both visible and invisible; and the mean values bound the whole system together so intimately that no changes could be made in any part without changing the whole.

This unique way of working has resulted in a table of wavelengths from 2100 to 7700 whose accuracy might be estimated as follows:-

Distribute less than $\frac{1}{100}$ division of Ångström properly throughout the table as a correction, and it will become perfect within the limits 2400 and 7000 .

The above is only a sketch of the methods used. The complete details of the work are ready for publication, but I have not yet found any journal or society willing to undertake it.

\section{Description of the Table.}

The first column gives the name of the element whose wave-length has been measured. If a letter stands at the left, it is the "name" of the line in the solar spectrum. A(o) 
and $\mathrm{A}(\mathrm{wv})$ denote lines due to absorption by the oxygen and the water-vapour, respectively, in the earth's atmosphere. An ? mark after an element means that it is doubtful if the line is really due to the element named. If two elements are given on the same line (e.g. Mn-Di w.l. 3295.957), it is to be understood that they have apparently coinciding lines at that particular wave-length. If two or more elements are bracketed

$$
\text { e.g., } \left.\begin{array}{l}
\mathrm{Mn} \\
\mathrm{Fi}_{\theta}
\end{array}\right\} w .1 .5260 \cdot 384,
$$

it means that the first one has a line coinciding with one side of the corresponding line in the solar spectrum, the second one has a line coinciding with the middle, \&c., and the appearance of the solar line itself is given in a later column. An ? standing alone denotes that the element which corresponds to the given wave-length is unknown.

The second column gives the intensity of the line in the arc spectrum; the third its appearanee, and the fourth and fifth do the same for the line in the solar spectrum. $R$ stands for "reversed ;" N, nebulous ; d, double ; t, triple ; ?, doubtful or difficult. The size of the number indicates to some extent the intensity of the line. For instance the intensity 10 means that the line is apparently 10 times as intense as the intensity 1. Measurements of intensity by eye-observations, direct or on photographic plates, are of course most uncertain. And so the figures given are estimates which do not apply to comparisons of different portions of the spectrum, but are intended to give some idea of the relative effects. The intensity of some lines in the arc spectrum of a given substance, e.g. $\mathrm{Ca}$, is often so much greater than that of the others, that the absence of some lines in the solar spectrum is easily understood. The sixth column gives the character of the standard. $M$ means that the line is a standard in the arc spectrum; $\odot$ means that the line is an ordinary solar standard; $\odot^{\prime}$, a better solar standard; $\odot^{\prime \prime}$, a remarkably good solar standard; and $\odot_{1}$ a rather poor solar standard.

The next two columns give the "weights" to be attached to the values of the wave-lengths as standards in the are and solar spectra, respectively.

The last two columns give the final values of the wavelengths measured in Ångström units, $i . e_{\text {. }}$, in ten millionths of a millimetre in ordinary air at about $20^{\circ} \mathrm{C}$. and $760 \mathrm{millim}$. pressure.

Notes marked J. are by Mr. Jewell. 


\begin{tabular}{|c|c|c|c|c|c|c|c|c|c|}
\hline \multirow{2}{*}{ Elements } & \multicolumn{2}{|c|}{ IN ARC. } & \multicolumn{2}{|c|}{ In SUN. } & \multirow{2}{*}{$\begin{array}{c}\text { Kind } \\
\text { of } \\
\text { Stand- } \\
\text { ard. }\end{array}$} & \multicolumn{2}{|c|}{ Whight. } & \multirow{2}{*}{$\begin{array}{c}\text { Wave- } \\
\text { length } \\
\text { in Arc. }\end{array}$} & \multirow{2}{*}{$\begin{array}{l}\text { Ware- } \\
\text { length } \\
\text { in Sun. }\end{array}$} \\
\hline & $\begin{array}{l}\text { Inten- } \\
\text { sity. }\end{array}$ & $\begin{array}{c}\text { Appear- } \\
\text { ance. }\end{array}$ & $\begin{array}{l}\text { Inten- } \\
\text { sity. }\end{array}$ & $\begin{array}{c}\text { Appear- } \\
\text { ance. }\end{array}$ & & In & $\underset{\text { Sun. }}{\text { In }}$ & & \\
\hline & 2 & $\cdots$ & $\cdots$ & $\cdots$ & $\mathbf{M}$ & 1 & $\cdots$ & $2152 \cdot 912$ & \\
\hline $\mathbf{S r} \ldots$ & 3 & $\ldots$ & $\ldots$ & $\ldots$ & $\mathbf{M}$ & 1 & $\ldots$ & $|2165.990|$ & \\
\hline $\begin{array}{ll}\mathrm{Si} & \ldots . . .\end{array}$ & 2 & $\ldots$ & $\ldots$ & $\ldots$ & $\mathbf{M}$ & 2 & $\ldots$ & 2208.060 & \\
\hline 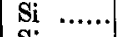 & 3 & $\cdots$ & $\cdots$ & ... & $\mathbf{M}$ & 2 & $\ldots$ & 2210.939 & \\
\hline $\mathrm{Si}_{\mathrm{Si}} \cdot \ldots . .$. & 2 & ... & $\cdots$ & $\cdots$ & $M$ & 2 & $\cdots$ & $|2211 \cdot 759|$ & \\
\hline $\begin{array}{cc}\mathrm{Si} & \ldots . . . \\
\mathrm{Si} & \cdots\end{array}$ & 4 & $\cdots$ & $\cdots$ & $\cdots$ & $\mathbf{M}$ & 2 & ... & $|2216760|$ & \\
\hline $\begin{array}{ccc}\mathrm{S1} & \ldots . . . \\
\mathrm{Al} & \ldots . . .\end{array}$ & $\begin{array}{l}2 \\
3\end{array}$ & $\cdots$ & $\cdots$ & $\cdots$ & $\frac{\mathbf{M}}{\mathbf{M}}$ & $\begin{array}{l}2 \\
2\end{array}$ & $\cdots$ & $\left|\begin{array}{l}2218 \cdot 146 \\
2263 \cdot 507\end{array}\right|$ & \\
\hline Al ....... & 4 & $\cdots$ & ... & $\begin{array}{l}\cdots \\
\ldots\end{array}$ & $M$ & 2 & $\begin{array}{l}\cdots \\
\ldots\end{array}$ & $\left|\begin{array}{l}22000 \\
2269 \cdot 161\end{array}\right|$ & \\
\hline Sr ....... & 10 & $\mathbf{R}$ & $\ldots$ & $\cdots$ & $\mathbf{M}$ & 1 & .... & $2275 \cdot 376$ & \\
\hline $\mathrm{Ca} \ldots . . .$. & 20 & $\mathbf{R}$ & $\ldots$ & $\ldots$ & $\mathbf{M}$ & 3 & $\ldots$ & $2275 \cdot 602$ & \\
\hline Fe? ... & & 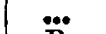 & $\cdots$ & $\ldots$ & M & 2 & $\ldots$ & $|2298 \cdot 246|$ & \\
\hline $\mathrm{Ba} \ldots . .$. & 20 & $\mathbf{R}$ & $\cdots$ & $\ldots$ & $\mathbf{M}$ & 1 & $\cdots$ & $2304 \cdot 364$ & \\
\hline $\begin{array}{l}\mathrm{Ba} \\
\mathrm{Fe}\end{array}$ & 20 & $\boldsymbol{R}$ & $\ldots$ & $\ldots$ & $\mathbf{M}$ & 1 & ... & $2335 \cdot 267$ & \\
\hline $\begin{array}{ll}\mathrm{Fe} & . . . . \\
\mathrm{Fe} & . . .\end{array}$ & $\cdots$ & $\cdots$ & $\cdots$ & $\cdots$ & $\mathbf{M}$ & 2 & $\cdots$ & $2343 \cdot 571$ & \\
\hline $\begin{array}{l}\mathrm{Fe} \\
\mathrm{Fe}\end{array}$ & $\cdots$ & $\cdots$ & $\cdots$ & $\ldots$ & $\frac{M}{M}$ & $\begin{array}{l}2 \\
2\end{array}$ & $\begin{array}{l}\cdots \\
\ldots\end{array}$ & $\begin{array}{l}2348 \cdot 385 \\
2364 \cdot 897\end{array}$ & \\
\hline Al .......... & $\dddot{6}$ & $\cdots$ & $\cdots$ & $\cdots$ & $\mathrm{M}$ & 3 & $\cdots$ & $2367 \cdot 144$ & \\
\hline Al ...... & 7 & $\ldots$ & $\cdots$ & $\ldots$ & $\mathbf{M}$ & 3 & $\ldots$ & $2373 \cdot 213$ & \\
\hline$F_{\theta} \ldots . .$. & $\cdots$ & $\cdots$ & $\ldots$ & $\ldots$ & $\mathbf{M}$ & 2 & $\ldots$ & $2373 \cdot 771$ & \\
\hline $\mathrm{Fe} ? \ldots$ & $\ldots$ & $\ldots$ & $\cdots$ & $\ldots$ & $\mathbf{M}$ & 3 & ... & $2382 \cdot 122$ & \\
\hline $\mathrm{Fe} \ldots \ldots$ & $\cdots$ & $\cdots$ & $\ldots$ & $\ldots$ & $\mathbf{M}$ & 2 & $\ldots$ & $2388 \cdot 710$ & \\
\hline Fe? $\ldots$ & $\dddot{2}$ & $\dddot{m}$ & $\cdots$ & $\ldots$ & $\mathbf{M}$ & 3 & $\cdots$ & $2395 \cdot 715$ & \\
\hline $\mid \begin{array}{ll}\mathrm{Ca} \\
\mathrm{Fe}\end{array}$ & $\begin{array}{l}25 \\
\ldots\end{array}$ & $\mathbf{R}$ & $\cdots$ & $\cdots$ & $\mathbf{M}$ & 5 & $\cdots$ & $2398 \cdot 667$ & \\
\hline $\mid \begin{array}{ccc}\mathrm{Fe} & \ldots . . . \\
\mathrm{Fe} & \ldots . . . .\end{array}$ & $\begin{array}{l}\cdots \\
\cdots\end{array}$ & 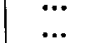 & $\begin{array}{l}\cdots \\
\cdots\end{array}$ & $\begin{array}{l}\ldots \\
\ldots\end{array}$ & $\frac{\mathbf{M}}{\mathbf{M}}$ & $\begin{array}{l}2 \\
2\end{array}$ & $\cdots$ & $\begin{array}{l}2399 \cdot 328 \\
2404 \cdot 971\end{array}$ & \\
\hline $\mathrm{Fe} \ldots$ & $\ldots$ & $\cdots$ & $\cdots$ & $\cdots$ & $M$ & 2 & $\cdots$ & 2406743 & \\
\hline $\mathrm{Fe} \ldots \ldots$ & $\cdots$ & $\cdots$ & $\ldots$ & ... & $\mathbf{M}$ & 2 & $\ldots$ & $2410 \cdot 604$ & \\
\hline $\mid \begin{array}{cc}\mathrm{Si} & \ldots . . . .\end{array}$ & 8 & $\cdots$ & $\ldots$ & $\cdots$ & M & 15 & $\ldots$ & $2435 \cdot 247$ & \\
\hline $\mid \begin{array}{cc}\mathbf{S i} & \ldots . . .\end{array}$ & 3 & $\cdots$ & $\cdots$ & $\cdots$ & $\mathbf{M}$ & 10 & $\ldots$ & 2438.864 & \\
\hline $\mathrm{Si}$ & 3 & $\cdots$ & $\cdots$ & $\cdots$ & $\mathbf{M}$ & 10 & ... & 2443.460 & \\
\hline $\mathrm{Fe} ?$ & $\because$ & $\cdots$ & $\cdots$ & $\cdots$ & $\mathbf{M}$ & 3 & $\cdots$ & $2447 \cdot 785$ & \\
\hline $\mathrm{Si}, \ldots . .$. & 3 & $\cdots$ & $\cdots$ & $\cdots$ & $\mathbf{M}$ & 10 & $\cdots$ & $2452 \cdot 219$ & \\
\hline $\begin{array}{lll}\mathrm{Fe} & ? \\
\mathrm{Fe} & \ldots & \ldots . . .\end{array}$ & $\ldots$ & $\cdots$ & $\cdots$ & $\cdots$ & $\stackrel{\mathbf{M}}{\mathbf{M}}$ & $\begin{array}{l}3 \\
3\end{array}$ & $\cdots$ & $\left|\begin{array}{l}2457 \cdot 680 \\
2462 \cdot 743\end{array}\right|$ & \\
\hline $\mathrm{Fe} . . . .$. & $\cdots$ & $\ldots$ & $\cdots$ & $\ldots$ & $\mathbf{M}$ & $\mathbf{3}$ & $\cdots$ & 2472974 & \\
\hline$C^{*} \ldots$ & 10 & $\cdots$ & $\ldots$ & $\ldots$ & $\mathbf{M}$ & 15 & $\ldots$ & $2478 \cdot 661$ & \\
\hline Fe ....... & ... & $\ldots$ & $\ldots$ & $\ldots$ & $\mathbf{M}$ & 3 & $\ldots$ & 2479871 & \\
\hline$F_{\theta} \ldots . .$. & $\ldots$ & $\cdots$ & $\cdots$ & $\ldots$ & M & 3 & $\ldots$ & $|2483.359|$ & \\
\hline $\mathrm{Fe} \ldots .$. & $\cdots$ & $\ldots$ & $\ldots$ & $\cdots$ & $\mathbf{M}$ & 3 & $\ldots$ & $2484 \cdot 283$ & \\
\hline $\mathrm{Fe} \ldots . .$. & $\cdots$ & $\cdots$ & $\cdots$ & $\ldots$ & $\mathbf{M}$ & 3 & $\ldots$ & $|2488 \cdot 238|$ & \\
\hline $\mathrm{Fe} \ldots . .$. & $\ldots$ & ... & $\ldots$ & $\ldots$ & $\mathbf{M}$ & 3 & $\ldots$ & $2489 \cdot 838$ & \\
\hline $\mathrm{Fe} \ldots . .$. & ... & $\cdots$ & $\cdots$ & $\ldots$ & $\mathbf{M}$ & 3 & $\ldots$ & $2490 \cdot 723$ & \\
\hline $\mathrm{Fe}$ & 15 & $\cdots$ & $\cdots$ & & $\mathbf{M}$ & 3 & $\ldots$ & $2491 \cdot 244$ & \\
\hline Bo ....... & 15 & $\ldots$ & $\ldots$ & $\ldots$ & $\mathbf{M}$ & 20 & $\ldots$ & $2496 \cdot 867$ & \\
\hline Bo ....... & 20 & $\cdots$ & $\cdots$ & $\cdots$ & M & 20 & ... & 2497.821 & \\
\hline$F \theta \ldots$ & $\cdots$ & $\ldots$ & $\ldots$ & $\ldots$ & M & 3 & $\ldots$ & $|2501 \cdot 223|$ & \\
\hline $\mathrm{Si}$ & 10 & $\ldots$ & $\cdots$ & $\cdots$ & M & 15 & $\ldots$ & $2506 \cdot 994$ & \\
\hline $\mathrm{Fe} \ldots \ldots$ & $\cdots$ & $\ldots$ & $\ldots$ & $\cdots$ & M & 3 & $\cdots$ & $|2510934|$ & \\
\hline $\mathbf{S i} \quad \ldots \ldots$ & 7 & $\ldots$ & $\ldots$ & $\cdots$ & M & 10 & $\ldots$ & $2514 \cdot 417$ & \\
\hline ..... & 15 & $\cdots$ & $\cdots$ & ... & $M$ & 7 & $\cdots$ & $2516 \cdot 210$ & \\
\hline & $\cdots$ & $\cdots$ & $\cdots$ & $\because \cdot$ & $\mathbf{M}$ & 3 & $\cdots$ & $2518 \cdot 188$ & \\
\hline
\end{tabular}

* This line seems to be the only single line of carbon, not belonging to a band in the arc spectrum. It was determined to belong to carbon by the spark spectrum. (R.) 


\begin{tabular}{|c|c|c|c|c|c|c|c|c|c|}
\hline \multirow{2}{*}{ Elements } & \multicolumn{2}{|c|}{ In $A$ RC. } & \multicolumn{2}{|c|}{ In Sur. } & \multirow{2}{*}{$\begin{array}{l}\text { Kind } \\
\text { of } \\
\text { Stand- } \\
\text { ard. }\end{array}$} & \multicolumn{2}{|c|}{ Weight. } & \multirow{2}{*}{$\begin{array}{l}\text { Wave- } \\
\text { length } \\
\text { in Arc. }\end{array}$} & \multirow{2}{*}{$\begin{array}{l}\text { Wave- } \\
\text { length } \\
\text { in Sun. }\end{array}$} \\
\hline & $\begin{array}{l}\text { Inten- } \\
\text { sity. }\end{array}$ & $\begin{array}{c}\text { Appear- } \\
\text { ance. }\end{array}$ & $\begin{array}{c}\text { Inten- } \\
\text { sity. }\end{array}$ & $\begin{array}{c}\text { Appear- } \\
\text { ance. }\end{array}$ & & $\begin{array}{l}\text { In } \\
\text { Arc. }\end{array}$ & $\begin{array}{l}\text { In } \\
\text { Sun. }\end{array}$ & & \\
\hline Si & 8 & $\ldots$ & $\cdots$ & $\cdots$ & $\mathbf{M}$ & 10 & $\ldots$ & $|2519 \cdot 297|$ & \\
\hline Fe .... & $\ldots$ & $\ldots$ & ... & $\ldots$ & M & 3 & $\ldots$ & 2522.948 & \\
\hline $\mathrm{Si} \ldots$ & 9 & $\ldots$ & $\cdots$ & $\ldots$ & M & 10 & $\ldots$ & $2524 \cdot 206$ & \\
\hline $\mathrm{Fe} \ldots .$. & & $\ldots$ & $\cdots$ & $\cdots$ & $\mathbf{M}$ & 3 & $\cdots$ & $2527 \cdot 530$ & \\
\hline $\mathrm{Si} \quad \ldots$ & 10 & $\cdots$ & $\cdots$ & $\cdots$ & M & $\mathbf{5}$ & $\cdots$ & 2528.599 & \\
\hline$F_{\theta} \ldots \ldots$ & & $\dddot{\mathbf{R}}$ & $\cdots$ & $\cdots$ & $\mathbf{M}$ & 3 & $\cdots$ & $2535 \cdot 699$ & \\
\hline $\begin{array}{ll}\mathrm{Hg}^{*} & \ldots \\
\mathrm{Fe} & . .\end{array}$ & $\begin{array}{c}50 \\
\ldots\end{array}$ & $\begin{array}{l}\mathbf{R} \\
\ldots\end{array}$ & $\ldots$ & $\cdots$ & $\begin{array}{l}\mathbf{M} \\
\mathbf{M}\end{array}$ & $\begin{array}{l}2 \\
3\end{array}$ & $\ldots$ & $\mid \begin{array}{l}2536 \cdot 648 \\
2541 \cdot 058\end{array}$ & \\
\hline $\begin{array}{l}\mathrm{Fe} \\
\mathrm{Fe}\end{array}$ & $\cdots$ & $\cdots$ & $\begin{array}{l}\cdots \\
\ldots\end{array}$ & $\cdots$ & $\mathbf{M}$ & 3 & $\cdots$ & $\mid$ & \\
\hline $\mathrm{Fe} \ldots$ & $\cdots$ & $\ldots$ & $\ldots$ & $\ldots$ & M & 2 & $\ldots$ & 2549704 & \\
\hline Al .... & 10 & $\ldots$ & ... & $\cdots$ & $M$ & $\mathbf{5}$ & $\ldots$ & 2568.085 & \\
\hline Al .... & 10 & $\ldots$ & $\cdots$ & $\ldots$ & M & 5 & $\ldots$ & $2575 \cdot 198$ & \\
\hline Mn...... & $\ldots$ & $\ldots$ & $\ldots$ & $\cdots$ & $\mathbf{M}$ & 2 & $\cdots$ & $2576 \cdot 195 \mid$ & \\
\hline Fe? & $\ldots$ & $\ldots$ & $\cdots$ & $\cdots$ & $\mathbf{M}$ & 2 & $\ldots$ & $2584 \cdot 629$ & \\
\hline $\mathrm{Fe} . . . .$. & $\ldots$ & $\cdots$ & $\cdots$ & ... & $\mathbf{M}$ & 2 & $\cdots$ & $2585^{\circ} 963 \mid$ & \\
\hline Mn...... & $\ldots$ & $\ldots$ & $\cdots$ & $\ldots$ & $\mathbf{M}$ & 2 & ... & $2593 \cdot 810$ & \\
\hline $\mathrm{Fe} . . . .$. & $\ldots$ & & $\ldots$ & $\ldots$ & M & 2 & $\ldots$ & $2598 \cdot 460$ & \\
\hline $\mathrm{Fe} \ldots . .$. & $\ldots$ & R & $\cdots$ & $\ldots$ & $\mathbf{M}$ & 3 & $\ldots$ & $2599 \cdot 494$ & \\
\hline $\mathrm{Fe} . . . .$. & $\ldots$ & $\ldots$ & $\ldots$ & $\ldots$ & M & 2 & $\ldots$ & 2611.965 & \\
\hline $\mathrm{Fe} \ldots . .$. & $\ldots$ & $\ldots$ & $\ldots$ & $\ldots$ & M & 3 & $\ldots$ & $2631 \cdot 125$ & \\
\hline i $\ldots$. & $\check{\mathbf{5}}$ & $\ldots$ & $\cdots$ & $\ldots$ & M & 7 & $\cdots$ & $2631 \cdot 392$ & \\
\hline $\mathrm{Fe} . . . .$. & .. & $\ldots$ & $\ldots$ & $\ldots$ & $\mathbf{M}$ & $\mathbf{3}$ & $\cdots$ & $2679 \cdot 148$ & \\
\hline $\mathrm{Fe} . . . .$. & $\cdots$ & $\cdots$ & $\cdots$ & $\ldots$ & $\mathbf{M}$ & 2 & $\ldots$ & $2706^{\circ} 684$ & \\
\hline $\mathrm{Fe}$ & $\ldots$ & $\ldots$ & $\cdots$ & $\ldots$ & M & $\begin{array}{c}\mathbf{3} \\
\mathbf{3}\end{array}$ & ... & $\mid 2719 \cdot 119$ & \\
\hline Fe ....... & $\cdots$ & $\cdots$ & $\cdots$ & $\cdots$ & $\mathbf{M}$ & 3 & $\ldots$ & 2720.989 & \\
\hline $\mathrm{Ca} .$. & 5 & $\ldots$ & $\ldots$ & $\cdots$ & $\mathbf{M}$ & 1 & $\cdots$ & $2721 \cdot 762$ & \\
\hline $\mathrm{Fe} \ldots$ & $\ldots$ & $\ldots$ & $\ldots$ & $\ldots$ & M & 3 & $\ldots$ & $2723 \cdot 668$ & \\
\hline $\mathrm{Fe} ?$ & $\ldots$ & $\ldots$ & $\cdots$ & $\ldots$ & M & 3 & $\ldots$ & 2733.673 & \\
\hline $\mathrm{Fe}$ ?: & $\ldots$ & $\ldots$ & $\ldots$ & $\ldots$ & M & 3 & $\ldots$ & $2737 \cdot 405$ & \\
\hline Fo .... & $\ldots$ & $\ldots$ & $\ldots$ & $\ldots$ & M & 3 & $\ldots$ & $2742 \cdot 485$ & \\
\hline $\mathrm{Fe} . . . .$. & $\ldots$ & $\ldots$ & $\ldots$ & $\ldots$ & $\mathbf{M}$ & 3 & ... & $2750 \cdot 237 \mid$ & \\
\hline $\mathrm{Fe} \ldots . .$. & $\ldots$ & $\ldots$ & $\ldots$ & $\ldots$ & $\mathbf{M}$ & 2 & $\ldots$ & $2755 \cdot 837$ & \\
\hline $\mathrm{Fe} . . . .$. & $\ldots$ & $\ldots$ & $\ldots$ & $\ldots$ & M & 3 & $\ldots$ & 2756.427 & \\
\hline Fe ...... & $\ldots$ & $\ldots$ & $\cdots$ & $\ldots$ & M & 2 & $\cdots$ & $2761 \cdot 876$ & \\
\hline $\mathrm{Fe} . . . .$. & $\ldots$ & $\ldots$ & $\ldots$ & $\ldots$ & $\mathbf{M}$ & 2 & $\ldots$ & $2762 \cdot 110$ & \\
\hline $\mathrm{Fe} \ldots . . .$. & $\ldots$ & ... & $\ldots$ & $\ldots$ & $\mathbf{M}$ & 2 & $\ldots$ & $2767 \cdot 630$ & \\
\hline $\mathrm{Fe} . . . .$. & $\ldots$ & 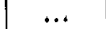 & $\ldots$ & $\ldots$ & M & 2 & $\ldots$ & $2772 \cdot 206$ & \\
\hline $\mathrm{Mg}{ }^{\dagger} \ldots$ & 5 & $\mathbf{R}$ & $\ldots$ & $\ldots$ & $\mathbf{M}$ & 5 & $\ldots$ & $2776798 \mid$ & \\
\hline $\mathrm{Fe} . . . .$. & $\ldots$ & & $\ldots$ & $\ldots$ & $\mathbf{M}$ & 2 & $\ldots$ & 2778.340 & \\
\hline $\mathrm{Mg} \dagger \ldots$ & 5 & $\mathbf{R}$ & $\ldots$ & $\ldots$ & M & $\mathbf{3}$ & $\ldots$ & $2778: 381$ & \\
\hline Mgt ... & 8 & $\mathrm{R}$ & $\ldots$ & $\ldots$ & $\mathbf{M}$ & 5 & $\ldots$ & $2779 \cdot 935 \mid$ & \\
\hline Mgt ... & 5 & $\mathbf{R}$ & $\ldots$ & $\cdots$ & M & 5 & $\ldots$ & $2781 \cdot 521$ & \\
\hline $\mathrm{Fe} \ldots \ldots$ & & & $\ldots$ & $\ldots$ & M & 1 & $\ldots$ & $2781 \cdot 945$ & \\
\hline Mgf & 5 & $\mathbf{R}$ & $\ldots$ & $\cdots$ & $\mathbf{M}$ & 5 & $\ldots$ & $|2783 \cdot 077|$ & \\
\hline $\mathrm{Fe} \ldots \ldots$ & $\ldots$ & ... & $\ldots$ & $\ldots$ & M & 3 & $\ldots$ & $2788 \cdot 201$ & \\
\hline Mn.. & & 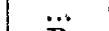 & & 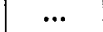 & M & 3 & $\ldots$ & $2794 \cdot 911$ & \\
\hline Mg..... & 20 & $\mathbf{R}$ & $\ldots$ & $\ldots$ & $\mathbf{M}$ & 12 & $\ldots$ & $|2795 \cdot 632|$ & \\
\hline $\mathrm{Mn} . . . .$. & $\cdots$ & ... & $\cdots$ & $\ldots$ & $\mathbf{M}$ & 3 & $\cdots$ & $2798 \cdot 369 \mid$ & \\
\hline Mn...... & 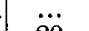 & $\dddot{0}$ & $\cdots$ & $\cdots$ & $\mathbf{M}$ & $\begin{array}{r}3 \\
10\end{array}$ & $\cdots$ & $2801 \cdot 183$ & \\
\hline Mg... & 20. & $\mathrm{R}$ & $\cdots$ & $\cdots$ & $\frac{\mathbf{M}}{\mathbf{M}}$ & 10 & $\cdots$ & \begin{tabular}{|c|}
$2802 \cdot 805$ \\
$2813 \cdot 388$
\end{tabular} & \\
\hline & 5 & $\cdots$ & $\cdots$ & $\cdots$ & $\frac{\mathrm{M}}{\mathrm{M}}$ & 1 & $\cdots$ & $\begin{array}{l}2013.080 \\
2823.389\end{array}$ & \\
\hline & 3 & $\cdots$ & $\cdots$ & $\cdots$ & & & & & \\
\hline
\end{tabular}

* This line appears as a shurp reversal, with no shading, in the spectra of all substances tried that contained any trace of cuntinuous spectrum in this rogion. (J.)

+ A remarkable symmetrical group of five lines in the spectrum of magnegium. 
Prof. H. A. Rowland on a

\begin{tabular}{|c|c|c|c|c|c|c|c|c|c|}
\hline \multirow{2}{*}{ Elements } & \multicolumn{2}{|c|}{ Is ARC. } & \multicolumn{2}{|c|}{ In Sun. } & \multirow{2}{*}{$\begin{array}{c}\text { Kind } \\
\text { of } \\
\text { Stand- } \\
\text { ard. }\end{array}$} & \multicolumn{2}{|c|}{ Wетант. } & \multirow{2}{*}{$\begin{array}{l}\text { Ware- } \\
\text { length. } \\
\text { in Aro. }\end{array}$} & \multirow{2}{*}{$\begin{array}{l}\text { Wave- } \\
\text { length } \\
\text { in Sun. }\end{array}$} \\
\hline & $\begin{array}{l}\text { Inten- } \\
\text { sity. }\end{array}$ & $\begin{array}{c}\text { Appoar- } \\
\text { ance. }\end{array}$ & $\begin{array}{l}\text { Inten- } \\
\text { sity. }\end{array}$ & $\begin{array}{c}\text { Appear- } \\
\text { ance. }\end{array}$ & & $\begin{array}{l}\text { In } \\
\text { Arc. }\end{array}$ & $\begin{array}{c}\text { In } \\
\text { Sun. }\end{array}$ & & \\
\hline Fe ......... & 5 & $\cdots$. & ... & & $\mathbf{M}$ & & & $2825 \cdot 667$ & \\
\hline$F_{e}$. & 4 & $\ldots$ & $\ldots$ & $\ldots$ & $\mathbf{M}$ & 7 & ... & 2832.545 & \\
\hline $\mathrm{Fe} \ldots . .$. & 3 & $\ldots$ & $\cdots$ & $\cdots$ & M & 1 & $\ldots$ & 2838.226 & \\
\hline $\mathrm{Fe} \ldots \ldots$ & 3 & $\ldots$ & $\ldots$ & $\cdots$ & $\mathbf{M}$ & 1 & $\ldots$ & $2843 \cdot 744$ & \\
\hline Fe $\ldots \ldots$ & 5 & $\ldots$ & $\ldots$ & $\ldots$ & M & 7 & $\ldots$ & 2844.085 & \\
\hline Fe ........ & 6 & $\ldots$ & $\ldots$ & $\cdots$ & M & 5 & $\ldots$ & $2851 \cdot 904$ & \\
\hline $\mathrm{Mg} \ldots \ldots$ & 100 & $\mathbf{R}$ & $\ldots$ & $\ldots$ & M & 15 & $\ldots$ & $2852 \cdot 239$ & \\
\hline $\mathrm{Si}_{\mathrm{i}} \ldots . .$. & 15 & & $\ldots$ & $\ldots$ & $\bar{M}$ & 12 & $\ldots$ & $2881 \cdot 695$ & \\
\hline $\mathrm{Fe} \ldots \ldots$ & 7 & $\mathbf{R}$ & .. & $\cdots$ & M & 3 & $\ldots$ & $2912 \cdot 275$ & \\
\hline$F e \ldots . .$. & 8 & $\mathbf{R}$ & $\ldots$ & $\ldots$ & M & 3 & $\ldots$ & $2929 \cdot 127$ & \\
\hline Fe ......... & 10 & $\mathbf{R}$ & $\ldots$ & $\ldots$ & M & 4 & $\ldots$ & $2937 \cdot 020 \mid$ & \\
\hline Fe ........ & 8 & $\mathbf{R}$ & $\ldots$ & $\ldots$ & $\mathbf{M}$ & 4 & $\ldots$ & 2947.993 & \\
\hline $\mathrm{Fe} \ldots \ldots$ & 7 & $\mathrm{R}$. & .. & $\ldots$ & M & 4 & $\ldots$ & $2954 \cdot 058$ & \\
\hline $\mathrm{Fe} \ldots \ldots$ & 5 & $\ldots$ & $\ldots$ & $\ldots$ & M & 3 & $\ldots$ & $2957 \cdot 485$ & \\
\hline $\mathrm{Fe} \ldots . .$. & 5 & $\cdots$ & $\ldots$ & $\ldots$ & M & 3 & ... & $2965 \cdot 381$ & \\
\hline Fe ........... & $\because$ & $\mathbf{P}$ & $\cdots$ & $\ldots$ & M & 1 & $\ldots$ & 2966.985 & \\
\hline Fo ......... & 8 & $\mathbf{R}$ & $\cdots$ & $\cdots$ & M & 12 & $\ldots$ & $2967 \cdot 016$ & \\
\hline $\mathrm{F} \theta \ldots . .$. & 4 & $\mathbf{R}$ & $\ldots$ & $\ldots$ & $\mathbf{M}$ & 7 & $\ldots$ & $2970 \cdot 223$ & \\
\hline Fe ........ & 6 & $\mathbf{R}$ & $\ldots$ & $\ldots$ & M & 7 & $\ldots$ & $2973 \cdot 254$ & \\
\hline Fe $\ldots \ldots$ & 12 & $\mathbf{R}$ & $\ldots$ & $\cdots$ & M & 15 & $\ldots$ & $2973 \cdot 358$ & \\
\hline Fe ... & 2 & & .. & $\ldots$ & $M$ & 6 & $\cdots$ & 2981.570 & \\
\hline $\mathrm{Fe} \ldots . .$. & 10 & $\mathrm{R}$ & $\ldots$ & $\ldots$ & M & 15 & $\ldots$ & $2983 \cdot 689 \mid$ & \\
\hline Fe $\ldots . .$. & $\because$ & $\cdots$ & $\ldots$ & $\ldots$ & M & 1 & $\cdots$ & $2987 \cdot 410$ & \\
\hline $\mathrm{Si} \quad \ldots . .$. & 4 & $\dddot{n}$ & $\ldots$ & $\ldots$ & M & 5 & $\ldots$ & $2987 \cdot 766$ & \\
\hline Fo ............ & 8 & $\mathbf{R}$ & $\cdots$ & $\cdots$ & M & 18 & $\ldots$ & 2994.547 & \\
\hline $\mathrm{Ca} \ldots .$. & 7 & $\mathbf{R}$ & $\ldots$ & $\ldots$ & M & 3 & $\ldots$ & 2995074 & \\
\hline $\mathrm{Ca} \ldots \ldots$. & 10 & $\mathbf{R}$ & $\ldots$ & $\ldots$ & M & 3 & $\ldots$ & $2997 \cdot 430$ & \\
\hline $\mathrm{Fe} \ldots \ldots$ & 4 & $\mathbf{R}$ & $\cdots$ & $\ldots$ & $\mathbf{M}$ & 5 & ... & $2999 \cdot 632$ & \\
\hline $\mathrm{Ca} \ldots . .$. & 6 & $\mathbf{R}$ & $\ldots$ & $\ldots$ & M & 3 & $\ldots$ & $2999 \cdot 767$ & \\
\hline $\mathrm{Ca} \ldots \ldots$ & 8 & $\mathrm{R}$ & $\ldots$ & $\ldots$ & M & 3 & $\ldots$ & $3000 \cdot 976$ & \\
\hline Fo ....... & 8 & $\mathrm{R}$ & $\cdots$ & $\ldots$ & M & 15 & $\ldots$ & 3001.070 & \\
\hline$?+\ldots .$. & $\ldots$ & $\cdots$ & 3 & $\cdots$ & $\odot$ & 1 & $\ldots$ & $\ldots \ldots$ & $3005 \cdot 160$ \\
\hline$?$ & & & 4 & $\ldots$ & $\odot$ & 1 & $\ldots$ & ${ }^{3}$ & $3005 \cdot 40$ \\
\hline $\mathrm{Ca} \ldots \ldots$ & 15 & $\mathbf{R}$ & $\cdots$ & $\ldots$ & $\bar{M}$ & 3 & $\ldots$ & 3006.978 & \\
\hline $\mathrm{Fe} \ldots . .$. & 2 & $\ldots$ & $\ldots$ & $\ldots$ & M & 1 & $\ldots$ & $3007 \cdot 260$ & \\
\hline $\mathrm{Fe}$ & 1 & & $\cdots$ & $\ldots$ & M & 3 & $\ldots$ & $3007 \cdot 408$ & \\
\hline $\mathrm{Fe}$ & 6 & $\mathbf{R}$ & $\ldots$ & $\ldots$ & $\bar{M}$ & 15 & $\ldots$ & $3008 \cdot 255$ & \\
\hline $\mathrm{Ca} \ldots \ldots$ & 7 & $\mathbf{R}$ & $\ldots$ & $\ldots$ & $\bar{M}$ & 3 & $\ldots$ & $|3009 \cdot 327|$ & \\
\hline Fe ........ & 4 & $\mathbf{R}$ & $\cdots$ & $\cdots$ & M & 3 & $\ldots$ & $3009 \cdot 696$ & \\
\hline$? \ldots \ldots$ & $\cdots$ & $\cdots$ & 4 & $\dddot{\partial}$ & $\bigodot^{\prime}$ & 5 & $\ldots$ & ..... & $3012: 557$ \\
\hline ? $\cdots \cdots$ & $\cdots$ & $\cdots$ & 6 & d? & $\bigodot^{\prime}$ & 4 & $\ldots$ & & $3014 \cdot 274$ \\
\hline Fe ....... & $\dddot{*}$ & $\cdots$ & 3 & $\cdots$ & $\bar{M}$ & 1 & $\ldots$ & $3016 \cdot 296$ & \\
\hline Fe ....... & 5 & $\cdots$ & $\cdots$ & $\ldots$ & M & 1 & $\ldots$ & $3017 \cdot 747$ & \\
\hline Fe ....... & $\mathbf{5}$ & $\ldots$ & ... & $\ldots$ & M & 1 & $\ldots$ & $\mid 3019 \cdot 109$ & \\
\hline Fe ....... & 프 & $\dddot{m}$ & $\cdots$ & $\cdots$ & M & 1 & $\ldots$ & $3019 \cdot 752$ & \\
\hline Fe ....... & 10 & $\mathbf{R}$ & $\ldots$ & $\ldots$ & MI & 15 & $\ldots$ & $3020 \cdot 611$ & \\
\hline $\mathrm{Fe} \ldots . .$. & 25 & $\mathbf{R}$ & $\cdots$ & $\ldots$ & M & 18 & $\ldots$ & $3020 \cdot 759 \mid$ & \\
\hline Fe $\ldots . .$. & 15 & $\mathbf{R}$ & 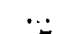 & $\ldots$ & M & 18 & $\ldots$ & $3021 \cdot 191$ & \\
\hline $\mathrm{Fe} \ldots . .$. & 7 & $\mathbf{R}$ & 7 & $\cdots$ & M & 7 & $\ldots$ & $3024 \cdot 154$ & \\
\hline$?+\cdots .$. & $\ldots$ & $\ldots$ & $\begin{array}{l}5 \\
4\end{array}$ & $\cdots$ & $\odot^{\prime}$ & $\cdots$ & 7 & ...... & $3024 \cdot 475$ \\
\hline $\mathrm{Fe}$ & 10̈0 & $\mathbf{R}$ & $\begin{array}{r}4 \\
10\end{array}$ & $\begin{array}{l}\cdots \\
\ldots\end{array}$ & $\stackrel{\oplus}{M}$ & 7 & $\begin{array}{c}7 \\
\ldots\end{array}$ & $3025 \cdot 958$ & \\
\hline $\mathrm{Fe} . . . .$. & $\cdots$ & $\cdots$ & $\cdots$ & $\cdots$ & $\mathbf{M}$ & 1 & $\cdots$ & $3027 \cdot 245$ & \\
\hline ? $\cdots . .$. & & & 5 & $\cdots$ & $\odot$ & 7 & $\ldots$ & & 3035.850 \\
\hline Fo ......... & 15 & $\mathbf{R}$ & 15 & $\cdots$ & $\mathbf{M}$ & 10 & 2 & 3037.505 & $3037 \cdot 492$ \\
\hline
\end{tabular}


New Table of Standard Wave-lengths.

\begin{tabular}{|c|c|c|c|c|c|c|c|c|c|}
\hline \multirow{2}{*}{ Elements } & \multicolumn{2}{|c|}{ IN ARC. } & \multicolumn{2}{|c|}{ IN Sun. } & \multirow{2}{*}{$\begin{array}{c}\text { Kind } \\
\text { of } \\
\text { Stand- } \\
\text { ard. }\end{array}$} & \multicolumn{2}{|c|}{ WhIG } & \multirow{2}{*}{$\begin{array}{l}\text { Wave- } \\
\text { length } \\
\text { in Arc. }\end{array}$} & \multirow{2}{*}{$\begin{array}{l}\text { Wave- } \\
\text { length } \\
\text { in Sun. }\end{array}$} \\
\hline & $\begin{array}{c}\text { Inten- } \\
\text { sity. }\end{array}$ & $\begin{array}{c}\text { Appear- } \\
\text { ance. }\end{array}$ & $\begin{array}{l}\text { Inten- } \\
\text { sity. }\end{array}$ & $\begin{array}{c}\text { Appear- } \\
\text { ance. }\end{array}$ & & $\begin{array}{c}\text { In } \\
\text { Arc. }\end{array}$ & $\begin{array}{c}\text { In } \\
\text { Sun. }\end{array}$ & & \\
\hline $\mathrm{Ca}$. & 15 & $\mathbf{R}$ & 4 & & $\mathbf{M} \odot_{1}$ & 3 & 2 & $3044^{\circ} 114$ & $3044 \cdot 119$ \\
\hline Mn. & 10 & $\mathbf{R}$ & 3 & ... & $\bigodot^{\prime}$ & 5 & $\ldots$ & $\ldots \ldots$ & \\
\hline$? \ldots$ & & & & & $\overbrace{}^{\prime}$ & 1 & ... & & $3046778 \mid$ \\
\hline $\mathrm{Fe} . . .$. & 20 & $\mathbf{R}$ & 20 & $\cdots$ & $\mathbf{M}$ & 13 & $\cdots$ & $3047 \cdot 720$ & \\
\hline ? & ... & $\cdots$ & $3\}$ & d & $\odot^{\prime \prime}$ & $\cdots$ & 5 & $\cdots \cdots$ & $3050 \cdot 212$ \\
\hline $\mathrm{Fe} . . . .$. & $\cdots$ & $\cdots$ & 3 & $\ldots$ & $\odot^{\prime}$ & $\cdots$ & 5 & ...... & $3053 \cdot 173$ \\
\hline$? \quad \ldots .$. & $\cdots$ & $\cdots$ & $\left.\begin{array}{l}3 \\
3\end{array}\right\}$ & $d$ & $\odot^{\prime}$ & $\cdots$ & 1 & ...... & $3053 \cdot 527$ \\
\hline$? \ldots$ & 政 & & 5 & $d ?$ & $\bigodot^{\prime \prime}$ & & 5 & & $3055 \cdot 821$ \\
\hline Fe $\ldots . .$. & 10 & $\mathbf{R}$ & 10 & $\ldots$ & $\bar{M}$ & 8 & $\ldots$ & $3057 \cdot 557$ & \\
\hline $\begin{array}{ccc}\mathrm{Fe} & \ldots . . . \\
? & \ldots & .\end{array}$ & 10 & $\mathrm{R}$ & $\begin{array}{r}10 \\
3\end{array}$ & $\begin{array}{l}\ldots \\
\ldots\end{array}$ & $\begin{array}{l}\mathbf{M} \\
\odot^{\prime}\end{array}$ & 15 & $\dddot{i}$ & $3059 \cdot 200$ & 3061.098 \\
\hline Co $\ldots . . .$. & .8 & $\mathbf{R}$ & 3 & $\ldots$ & $\mathbf{M} \odot \prime$ & $\ddot{1}$ & 5 & 3061.932 & 3061.930 \\
\hline $\mathrm{Fe} \ldots$ & 10 & $\mathbf{R}$ & 10 & ... & $M$ & 10 & $\ldots$ & $3067 \cdot 363$ & \\
\hline $\mathrm{Ti}$ & 6 & & 8 & $\ldots$ & M & 3 & $\ldots$ & $3075 \cdot 339$ & \\
\hline Fe .... & 10 & $\mathbf{R}$ & 10 & $\ldots$ & M & 4 & $\ldots$ & 3075.849 & \\
\hline $\mathrm{Fe}$. & $\ldots$ & $\ldots$ & 2 & $\ldots$ & M & 1 & $\cdots$ & $|3077 \cdot 216|$ & \\
\hline$?$ & $\cdots$ & $\cdots$ & 4 & $\ldots$ & $\odot$ & $\cdots$ & 6 & & $3077 \cdot 303$ \\
\hline $\mathrm{Fe}$ ? & $\cdots$ & $\ldots$ & 4 & $\ldots$ & $\bigodot^{\prime \prime}$ & $\cdots$ & 6 & & $3078 \cdot 148$ \\
\hline & 4 & $\cdots$ & 6 & $\cdots$ & M & 3 & & $|3078759|$ & \\
\hline Mn..... & 7 & $\cdots$ & 2 & $\ldots$ & $\bigodot^{\prime \prime}$ & .. & 1 & ...... & $3079 \cdot 724$ \\
\hline$?$ & 0 & 8 & 5 & $\ldots$ & $\bigodot^{\prime \prime}$ & & 1 & & 3080.863 \\
\hline Al ..... & 20 & $\mathbf{R}$ & 7 & $\ldots$ & M. & 17 & $\ldots$ & $3082 \cdot 272$ & \\
\hline $\mathrm{Fe}$. & 6 & R & 7 & $\ldots$ & M & 5 & 7 & 3083.849 & \\
\hline & & & 4 & $\ldots$ & $\bigodot^{\prime \prime}$ & 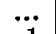 & 1 & & 3086.891 \\
\hline $\mathrm{Ti}$ & 8 & $R$ & 8 & $\ldots$ & $\overline{\mathbf{M}}$ & 1 & $\cdots$ & 3088.137 & \\
\hline Al . & 20 & $\mathbf{R}$ & 10 & $\ldots$ & M & 15 & ... & $3092 \cdot 824$ & \\
\hline Al . & 4 & $\cdots$ & 2 & $\ldots$ & M & 8 & $\ddot{0}$ & $3092 \cdot 962$ & \\
\hline & 1 & $\cdots$ & 2 & $\ldots$ & $\odot^{\prime}$ & $\ldots$ & 9 & ....... & $3094 \cdot 739$ \\
\hline $\mathrm{Fe}$. & 1 & $\ldots$ & 3 & $\ldots$ & $\bigodot^{\prime}$ & $\cdots$ & 9 & & $3095.003 \mid$ \\
\hline $\mathrm{Fe} . . .$. & 4 & $\cdots$ & 7 & $\ldots$ & $\mathbf{M}$ & 3 & $\ldots$ & $3100 \cdot 064$ & \\
\hline $\mathrm{Fe}(\mathbf{M n})$ & $4-?$ & ... & 4 & $\ldots$ & M & 3 & $\ldots$ & $3100 \cdot 41 \overline{0}$ & \\
\hline & 6 & & 6 & $\ldots$ & $\mathbf{M}$ & 3 & $\ldots$ & $|3100.779|$ & \\
\hline & 20 & $\mathbf{R}$ & 8 & $\ldots$ & $\mathbf{M}$ & 3 & $\ldots$ & $3101 \cdot 673$ & \\
\hline $\mathrm{Ni}$ & 10 & $\mathbf{R}$ & 6 & $\ldots$ & M & 3 & $\cdots$ & $|3101.994|$ & \\
\hline & $\cdots$ & $\ldots$ & 2 & $\ldots$ & $\stackrel{\rho}{\rho}$ & ... & 1 & ....... & $\mid 3106 \cdot 677$ \\
\hline Cr? ... & $\ldots$ & ... & 3 & $\cdots$ & $\bigodot^{\prime}$ & $\ldots$ & 1 & ...... & $\mid 3109 \cdot 434$ \\
\hline .... & $\pi$ & $\cdots$ & 2 & $\cdots$ & $\odot^{\prime \prime}$ & $\cdots$ & 3 & ...... & $|3115 \cdot 160|$ \\
\hline$\nabla a^{*}$ & 7 & $\ldots$ & 5 & $\ldots$ & $\bigodot^{\prime \prime}$ & $\cdots$ & 9 & ....... & $3121 \cdot 275$ \\
\hline & 3 & 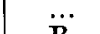 & 1 & $\cdots$ & $\bigodot^{\prime}$ & $\because$ & 5 & & $3129 \cdot 882$ \\
\hline & 10) & $\mathbf{R}$ & 8 & $\cdots$ & M & 1 & 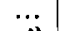 & $|3134 \cdot 223|$ & \\
\hline & 4 & $\cdots$ & 2 & $\cdots$ & $\bigodot^{\prime}$ & $\cdots$ & $\mathbf{3}$ & $\ldots \ldots$ & $3137 \cdot 441$ \\
\hline $\mathrm{Fe}$ & $\cdots$ & $\ldots$ & 3 & ... & $\bigodot^{\prime}$ & $\ldots$ & 5 & ....... & $3140 \cdot 869$ \\
\hline Fe & $\cdots$ & $\cdots$ & 2 & $\cdots$ & $\bigodot^{\prime}$ & $\cdots$ & 3 & & 3153870 \\
\hline & $\ldots$ & $\ldots$ & 8 & ... & M & 1 & 1 & 3158.994 & 315 \\
\hline M & ... & $\cdots$ & 1 & $\cdots$ & $\odot^{\prime}$ & $\ldots$ & 5 & $\ldots \ldots$ & $3167 \cdot 290 \mid$ \\
\hline Fe? ... & 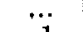 & 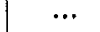 & 5 & $\cdots$ & $\bigodot^{\prime \prime}$ & $\cdots$ & 1 & $\ldots \ldots$ & $|3172 \cdot 175|$ \\
\hline $\mathrm{La}$ & 1 & 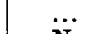 & 1 & $\ldots$ & $\odot^{\prime}$ & $\ldots$ & 5 & ....... & $|3176 \cdot 104|$ \\
\hline & 4 & $\mathbf{N}$ & 4 & $\cdots$ & $\bigodot^{\prime}$ & & 5 & & $3188 \cdot 164$ \\
\hline Nit & 3 & & 3 & $\ldots$ & $\mathbf{M}$ & 1 & 1 & 319572 & $3195 \cdot 702$ \\
\hline $\mathrm{Ti} \quad \ldots . .$. & 10 & R & 4 & $\ldots$ & $\mathbf{M} \odot$ & 1 & 5 & $3200 \cdot 040$ & $\mid 3200.032$ \\
\hline
\end{tabular}

* There is a very faint line on the violet side in the solar spectrum.

$t$ There is a line towards the red, also. 


\begin{tabular}{|c|c|c|c|c|c|c|c|c|c|}
\hline \multirow{2}{*}{ Elements } & \multicolumn{2}{|c|}{ IN ARC. } & \multicolumn{2}{|c|}{ In SUn. } & \multirow{2}{*}{$\begin{array}{l}\text { Kind } \\
\text { of } \\
\text { Stand. } \\
\text { ard. }\end{array}$} & \multicolumn{2}{|c|}{ W EIGHT. } & \multirow{2}{*}{$\begin{array}{l}\text { Wave- } \\
\text { length } \\
\text { in Arc. }\end{array}$} & \multirow{2}{*}{$\begin{array}{l}\text { Wave- } \\
\text { length } \\
\text { in Sun. }\end{array}$} \\
\hline & $\begin{array}{l}\text { Inten- } \\
\text { sity. }\end{array}$ & $\begin{array}{c}\text { Appear- } \\
\text { ance. }\end{array}$ & $\begin{array}{c}\text { Inten- } \\
\text { sity. }\end{array}$ & $\begin{array}{c}\text { Appear- } \\
\text { ance. }\end{array}$ & & $\begin{array}{c}\text { In } \\
\text { Arc. }\end{array}$ & \begin{tabular}{|l} 
In \\
Sun.
\end{tabular} & & \\
\hline Fe & 5 & & 5 & & M & 1 & $\cdots$ & $3214 \cdot 152$ & \\
\hline & 4 & .... & 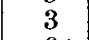 & $\ldots$ & $\odot^{\prime \prime}$ & $\ldots$ & 6 & $\ldots \ldots$ & $3218 \cdot 390$ \\
\hline $\mathrm{Fe}$ & $\ldots$ & $\ldots$ & 61 & $\ldots$ & $\odot^{\prime}$ & $\ldots$ & 1 & ....... & $3219 \cdot 697$ \\
\hline $\mathrm{Fe}$. & $\ldots$ & $\ldots$ & 5 & ... & $\odot^{\prime}$ & $\cdots$ & 1 & ....... & $3219 \cdot 909$ \\
\hline $\begin{array}{c}\mathrm{Fe} ? \\
?\end{array}$ & $\begin{array}{l}6 \\
?\end{array}$ & $\begin{array}{l}\ldots \\
\ldots\end{array}$ & $\left.\begin{array}{l}5 \\
7\end{array}\right\}$ & d & $\mathbf{M} \odot$ & 3 & 1 & $3222 \cdot 197$ & $3222 \cdot 203$ \\
\hline $\mathbf{T i}$ & 6 & $\ldots$ & 4 & $\ldots$ & $\odot "$ & & 3 & & 3224:368 \\
\hline $\mathrm{Fe}$ & 8 & $\cdots$ & 8 & $\cdots$ & $\mathbf{M}$ & 3 & 1 & $3225 \cdot 907 \mid$ & 322 \\
\hline $\begin{array}{c}\mathrm{Ti} \\
?\end{array}$ & $\left.\begin{array}{l}5 \\
?\end{array}\right\}$ & $\cdots$ & 5 & $\cdots$ & $\odot^{\prime \prime}$ & $\ldots$ & 1 & $\cdots \cdots \cdot$ & $3231 \cdot 421$ \\
\hline $\mathrm{Ti}$ & 6 & & 4 & $\cdots$ & $\stackrel{\odot}{\circ}$ & $\because$ & 12 & & $3232 \cdot 404$ \\
\hline Ti & 10 & $\mathbf{R}$ & 8 & $\cdots$ & M & 1 & 1 & $3236 \cdot 696$ & $3236 \cdot 697$ \\
\hline $\mathrm{Fe}$ & $\ldots$ & $\cdots$ & 6 & $\cdots$ & $\odot "$ & $\ldots$ & 12 & ...... & $3246^{\circ} 124$ \\
\hline $\mathrm{Cu}_{\mathrm{Mn}}$. & 40 & $\mathbf{R}$ & 9 & $\ldots$ & $\mathbf{M} \odot_{1}$ & 15 & 5 & $3247 \cdot 671$ & $3247 \cdot 680$ \\
\hline & $\begin{array}{l}4 \\
3 \\
3\end{array}$ & $\cdots$ & 4 & ... & $\odot^{\prime}$ & $\cdots$ & 10 & ...... & $3260 \cdot 384$ \\
\hline $\mathbf{F e}$ & 11 & & 4 & & $\odot^{\prime \prime}$ & & 10 & & \\
\hline $\begin{array}{l}\mathrm{Va} \\
\mathrm{Cu} \text {. }\end{array}$ & $\begin{array}{l}10 \\
30\end{array}$ & $\mathbf{R}$ & $\begin{array}{l}4 \\
6\end{array}$ & $\cdots$ & $\dot{\mathbf{M}} \odot{ }^{\prime \prime}$ & $\dddot{i 5}$ & $\begin{array}{r}10 \\
5\end{array}$ & $|3274 \cdot 090|$ & $\begin{array}{l}326 \\
327\end{array}$ \\
\hline & 6 & $\cdots$ & 5 & ... & $\odot^{\prime \prime}$ & $\ldots$ & 9 & $\mid \begin{array}{c}021 \pm 000 \\
\ldots . . .\end{array}$ & $3287 \cdot 791$ \\
\hline $\mathrm{Fe}$ & 5 & ... & 5 & $d ?$ & $\odot^{\prime \prime}$ & e & 10 & $\ldots$. & $3292 \cdot 174$ \\
\hline $\mathrm{Co}-$ & 47 & $\cdots$ & & & $\Omega^{\prime \prime}$ & $\cdots$ & & & \\
\hline Mn-Di & 32 & $\dddot{\mathrm{R}}$ & $\begin{array}{l}4 \\
6\end{array}$ & $\ldots$ & $\dot{M}_{\odot}^{\prime \prime}$ & $\ddot{i}$ & 9 & & 3295.957 \\
\hline $\begin{array}{l}\mathrm{Na} \\
\mathrm{Na}^{*}\end{array}$ & $\begin{array}{l}15 \\
10\end{array}$ & $\mathbf{R}$ & $\begin{array}{l}0 \\
5\end{array}$ & $\cdots$ & $\mathbf{M} \odot_{1}$ & $\begin{array}{l}1 \\
1\end{array}$ & $\begin{array}{l}6 \\
6\end{array}$ & $\begin{array}{l}3302 \cdot 504 \\
3303 \cdot 119\end{array}$ & $\begin{array}{l}330 \\
330\end{array}$ \\
\hline$?$ & $\cdots$ & .. & $3\}$ & d & $\odot^{\prime}$ & 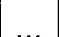 & 10 & & $\mid \begin{array}{l}3503101 \\
3303 \cdot 648\end{array}$ \\
\hline $\mathrm{Fe}$ & $\dddot{*}$ & $\cdots$ & $3\}$ & & & $\cdots$ & 10 & ….. & $3303 \cdot 648$ \\
\hline Fet & 10 & $\cdots$ & 7 & $\cdots$ & $\mathbf{M} \odot \odot_{1}$ & 1 & 5 & $3306^{\circ} \cdot 119$ & $3306 \cdot 117$ \\
\hline $\begin{array}{ll}\text { Fet } & \ldots \\
\text { Mn...j }\end{array}$ & 10 & $\cdots$ & 7 & $\cdots$ & $\mathbf{M} \odot_{1}$ & 1 & 5 & $3306^{\circ} 481$ & 330 \\
\hline$\left.\underset{\mathrm{Co}-\mathrm{Ti}_{i}}{\mathrm{Mn}}\right\}$ & $\begin{array}{c}2 \\
36\end{array}$ & $\ldots$ & $\left.\begin{array}{l}1 \\
4\end{array}\right\}$ & $d$ & $\odot^{\prime \prime}$ & $\cdots$ & 10 & ..... & 3308.928 \\
\hline & 5 & ... & 5 & $\ldots$ & $\bigodot^{\prime \prime}$ & $\ldots$ & 10 & ....... & $3318 \cdot 163$ \\
\hline $\mathbf{F}$ & 2 & ... & 2 & ... & $\odot^{\prime}$ & $\ldots$ & 8 & ...... & 333 \\
\hline & $\begin{array}{l}3 \\
3\end{array}$ & $\cdots$ & $\left.\begin{array}{l}3 \\
3\end{array}\right\}$ & d & $\odot^{\prime}$ & $\ldots$ & 9 & ..... & $3348 \cdot 011$ \\
\hline $\mathrm{Fe}, \ldots . . .$. & $\stackrel{5}{2}$ & $\cdots$ & 2 & $\ldots$ & $\odot^{\prime}$ & $\ldots$ & 9 & ...... & $3351 \cdot 877$ \\
\hline & 4 & $\ldots$ & 1 & $\cdots$ & $\odot^{\prime \prime}$ & $\ldots$ & 8 & ...... & $3356 \cdot 222$ \\
\hline $\mathrm{Ti}$ & $\begin{array}{l}5 \\
5\end{array}$ & $\ldots$ & $\begin{array}{l}3 \\
3\end{array}$ & d & $\odot^{\prime \prime}$ & $\cdots$ & 9 & $\ldots \ldots$ & $3377 \cdot 667$ \\
\hline $\mathrm{Fe} . . . .$. & 2 & $\cdots$ & 2 & $\ldots$ & $\odot^{\prime \prime}$ & 1 & 12 & $3389 \cdot 913$ & $3389 \cdot 887$ \\
\hline ... & 10 & $\mathbf{R}$ & $\begin{array}{l}3 \\
3\end{array}$ & d & $\odot^{\prime}$ & 1 & 12 & $3405 \cdot 255$ & $3405^{\circ} 272$ \\
\hline $\mathrm{Fe} \ldots . . .$. & .1 & $\ldots$ & 1 & $\ldots$ & $\odot^{\prime}$ & 1 & 18 & $3406 \cdot 602$ & 340 \\
\hline & 5 & $\ldots$ & 4 & $\cdots$ & $\odot^{\prime}$ & 1 & 18 & $3406 \cdot 965$ & 3406.955 \\
\hline & & $\ldots$ & & ... & & & 15 & & $3425 \cdot 721$ \\
\hline $\mathrm{Fe}$ & 6 & 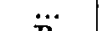 & 5 & $\cdots$ & $\mathbf{M}$ & 2 & 1 & $3427 \cdot 279$ & $3427 \cdot 282$ \\
\hline & 15 & $\boldsymbol{R}$ & 15 & $\cdots$ & $\mathbf{M} \odot_{1}$ & 7 & 4 & $3440 \cdot 756$ & $3440 \cdot 759$ \\
\hline
\end{tabular}

* Red component of a double which has a $\mathrm{Zn}$ line between. There is another Zn line at about 3302.7 in the solar spectrum.

+ Second line from red side of a group of five lines.

¥ Second line from violet side of a group of four lines.

$\$$ A very wide nebulous line of Ba comes here.

Red component of a double (the other line being also $\mathrm{Fe}$ ) having another fainter line at the red edge. 


\begin{tabular}{|c|c|c|c|c|c|c|c|c|c|}
\hline \multirow{2}{*}{ Elements } & \multicolumn{2}{|c|}{ Is ARc. } & \multicolumn{2}{|c|}{ In SUn. } & \multirow{2}{*}{$\begin{array}{l}\text { Kind } \\
\text { of } \\
\text { Stand } \\
\text { ard. }\end{array}$} & \multicolumn{2}{|c|}{ W EIGHT. } & \multirow{2}{*}{$\begin{array}{l}\text { Ware- } \\
\text { length } \\
\text { in Arc. }\end{array}$} & \multirow{2}{*}{$\begin{array}{l}\text { Ware- } \\
\text { length } \\
\text { in Sun. }\end{array}$} \\
\hline & $\begin{array}{l}\text { Inten- } \\
\text { sity. }\end{array}$ & $\begin{array}{l}\text { Appear- } \\
\text { ance. }\end{array}$ & $\begin{array}{c}\text { Inten } \\
\text { sity. }\end{array}$ & $\underset{\text { ance. }}{A p_{\text {ear }}}$ & & $\begin{array}{c}\text { In } \\
\text { Arc. }\end{array}$ & In & & \\
\hline $\mathrm{Fe}$ & 10 & & 10 & $\cdots$ & $\mathbf{M} \odot$ & 6 & 4 & $3441 \cdot 135$ & $3441 \cdot 1$ \\
\hline & 8 & R & 8 & $\ldots$ & $\mathbf{M} \odot$ & 6 & 4 & 3444.024 & \\
\hline Co ... & 6 & $\mathbf{R}$ & 4 & $\ldots$ & $\odot^{\prime \prime}$ & $\ldots$ & 10 & $\ldots$ & 3455.384 \\
\hline $\mathrm{Sr} ? \ldots$ & 8 & $\dddot{p}$ & 3 & $\ldots$ & $\odot^{\prime \prime}$ & $\cdots$ & 8 & $\ldots .$. & 346 \\
\hline Co $\}^{*}$ & 10 & $\stackrel{R}{R}$ & $\left.\begin{array}{l}4 \\
6\end{array}\right\}$ & $d$ & M & 7 & 3 & 3466.010 & 3465.991 \\
\hline $\begin{array}{l}\mathrm{Fe} \\
\text { Fot }\end{array}$ & $\begin{array}{l}10 \\
10\end{array}$ & $\mathbf{R}$ & $10^{\mathrm{J}}$ & $\cdots$ & $\mathbf{M}$ & 7 & 3 & 3475.602 & 3475.594 \\
\hline Fef ... & 7 & $\mathbf{R}$ & 8 & $\ldots$ & $\mathbf{M}$ & 5 & 2 & $3476 \cdot 848$ & 3476.831 \\
\hline $\mathrm{Fe}$ & 3 & $\cdots$ & 4 & $\cdots$ & $\odot^{\prime \prime}$ & $\ldots$ & 10 & ....... & 3478.001 \\
\hline $\mathrm{Ni}_{\mathrm{Ni}}$ & $2 J$ & & & & $\odot^{\prime}$ & & & & \\
\hline $\begin{array}{l}\mathrm{Ni} \\
\mathrm{Feg}\end{array}$ & $\begin{array}{c}4 \\
10 .\end{array}$ & $\begin{array}{l}\mathbf{R} \\
\mathbf{R}\end{array}$ & $\begin{array}{r}5 \\
10\end{array}$ & $\begin{array}{l}\cdots \\
\ldots\end{array}$ & $\stackrel{\odot}{M}$ & 7 & $\begin{array}{l}9 \\
3\end{array}$ & $3490 \cdot 724$ & $\begin{array}{l}3486036 \\
3490 \cdot 721\end{array}$ \\
\hline Oo & 4 & $\mathrm{R}$ & 4 & & $\odot^{\prime \prime}$ & & 8 & 04200 & $3491 \cdot 464$ \\
\hline & $?$ & $\ldots\}$ & $\mathbf{I}$ & $\cdots$ & & $\cdots$ & 0 & ...... & 3491464 \\
\hline $\mathrm{Fell}$ & 5 & $\mathbf{R}$ & 5 & $\cdots$ & $\mathbf{M}$ & 1 & 1 & $3497 \cdot 266$ & $3497 \cdot 264$ \\
\hline $\left.\begin{array}{c}F e \\
?\end{array}\right\}$ & $\begin{array}{l}6 \\
?\end{array}$ & $\boldsymbol{\kappa}$ & 3 & d & $\mathbf{M}$ & 5 & 4 & $3497 \cdot 991$ & $3497 \cdot 991$ \\
\hline $\mathrm{Fe} . . . .$. & 2 & & 3 & $\ldots$ & $\odot^{\prime \prime}$ & ... $>$ & 4 & ....... & $3500 \cdot 721$ \\
\hline $\mathrm{Ni} \ldots . .$. & 7 & $\mathbf{R}$ & 7 & $\ldots$ & $\odot^{\prime \prime}$ & $\ldots$ & 4 & ....... & 993 \\
\hline$T^{* *} \ldots$ & 5 & & 4 & $\ldots$ & $\ominus^{\prime}$ & 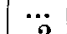 & 8 & & 3510.987 \\
\hline Fett ... & 7 & $R_{1}>$ & 6 & $\cdots$ & M & 2 & 3 & 3513.981 & \\
\hline Co ...... & 6 & $\mathbf{R}$ & 5 & $\cdots$ & $\stackrel{\odot}{ }^{\prime \prime}$ & $\because$ & 10 & 2 & 3518.487 \\
\hline $\mathrm{Tl}$ & 40 & $\mathbf{R}$ & ... & $\ldots$ & M & 1 & & $3519 \cdot 342$ & \\
\hline $\mathrm{Fe} \ldots$ & 5 & $\mathbf{R}$ & 7 & $\ldots$ & $\mathbf{M}$ & 6 & 5 & $3521 \cdot 409$ & $3521 \cdot 404$ \\
\hline Th . & 20 & $\mathbf{R}$ & ... & $\ldots$ & $\mathbf{M}$ & 1 & & 3529.547 & \\
\hline $\mathrm{Fe} . .$. & 3 & $\ldots$ & 5 & $\ldots$ & $\odot "$ & $\ldots$ & 10 & $\ldots \ldots$ & $3540 \cdot 266$ \\
\hline & $\cdots$ & $\cdots$ & 4 & $\ldots$ & $\odot^{\prime \prime}$ & $\because$ & 6 & & 35 \\
\hline Yt ... & 6 & ... & 2 & . & $\overline{\mathbf{M}} \vartheta^{\prime}$ & 1 & 1 & $3549 \cdot 147$ & $3549 \cdot 145$ \\
\hline $\mathrm{Fe} . . .$. & 2 & D & 3 & .. & $\odot^{n}$ & $\dddot{0}$ & 7 & & $3550 \cdot 006$ \\
\hline Fo ...... & 9 & $\mathbf{R}$ & 8 & $\ldots$ & $\mathbf{M} \odot$ & 3 & 4 & $3558 \cdot 674$ & $3558 \cdot 670$ \\
\hline $\left.\begin{array}{l}\mathrm{Ti} \\
\mathrm{Fe}\end{array}\right\} .$. & $\begin{array}{l}2 \\
1\end{array}$ & $\cdots$ & 4 & $d ?$ & $\odot^{\prime \prime}$ & $\cdots$ & 12 & ....... & $3564 \cdot 680$ \\
\hline $\mathrm{Fe} . . . .$. & $10^{\circ}$ & $\mathbf{R}$ & 12 & . & $\mathbf{M}$ & 6 & 4 & 3565.530 & 3565.528 \\
\hline $\mathrm{Fe} \ddagger \ddagger \ldots$ & 20 & $\mathbf{R}$ & 20 & & $\mathbf{M}$ & 8 & 4 & & \\
\hline $\mathrm{Fe} . . . .$. & 10 & & 10 & $\ldots$ & $\mathbf{M}$ & 1 & I & $3570 \cdot 412$ & 3570.402 \\
\hline .... & 30 & $\mathbf{R}$ & 40 & & $\mathbf{M} \odot_{1}$ & 9 & 6 & $3581 \cdot 344$ & $3581 \cdot 344$ \\
\hline $\mathrm{Fe}$ ?... & 2 & $\cdots$ & 4 & & $\odot$ & $\ldots$ & 12 & & 3583.483 \\
\hline Yt ...... & 6 & 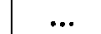 & 2 & $\ldots$ & $\mathbf{M}$ & 1 & 1 & $3584 \cdot 662$ & 3584.662 \\
\hline $0 \$ 8 .$. & 2 & $\ddot{*}>>>>$ & 1 & & M & 8 & $\cdots$ & 3585.992 & \\
\hline C $\ldots . .$. & $\cdots$ & $\cdots$ & 2 & $\cdots$ & M & 2 & $\ldots$ & 3586.041 & \\
\hline $\mathrm{C}\|\| .$. & . & . & 3 & .. & M & 7 & & $3590 \cdot 523$ & \\
\hline $\mathrm{Fe}$. & 5 & $\cdots$ & 4 & $\cdots$ & $\odot^{\prime \prime}$ & $\cdots$ & 12 & $\cdots \cdots$ & $|3597 \cdot 192|$ \\
\hline
\end{tabular}

* The metal measured was Fe.

$\uparrow$ Strongest line of group of six lines.

+ Also the strongest line in a group of six lines.

$\$$ There is a Co line near this towards the red.

Red component of a double.

IV Violet component of a double. Other component was not measured,

* A strong compound bismuth line also comes here.

t+ Violet component of a double.

tt Red component of a double. There is also another Fe line towards the red.

$\$ \&$ First line in the second head of the carbon band.

ill First line in the first head of carbon band. 


\begin{tabular}{|c|c|c|c|c|c|c|c|c|c|}
\hline \multirow{2}{*}{ en } & \multicolumn{2}{|c|}{ In ARc. } & \multicolumn{2}{|c|}{ Is Sus, } & \multirow{2}{*}{$\begin{array}{c}\text { Kind } \\
\text { of } \\
\text { Stand- } \\
\text { ard. }\end{array}$} & \multicolumn{2}{|c|}{ WeIGHT. } & \multirow{2}{*}{$\begin{array}{l}\text { Wave- } \\
\text { length } \\
\text { in Arc. }\end{array}$} & \multirow{2}{*}{$\begin{array}{l}\text { Wave- } \\
\text { length } \\
\text { in Sun. }\end{array}$} \\
\hline & $\begin{array}{l}\text { Inten- } \\
\text { sity. }\end{array}$ & Appear- & $\begin{array}{l}\text { Inten- } \\
\text { sity. }\end{array}$ & $\begin{array}{l}\text { Appear- } \\
\text { ance. }\end{array}$ & & In & $\begin{array}{l}\text { In } \\
\text { Sun. }\end{array}$ & & \\
\hline $\mathbf{Y t}$ & $10-?$ & ... $>$ & 4 & $\cdots$ & $\mathbf{M} \odot{ }^{\prime \prime}$ & 1 & 1 & 84 & 360 \\
\hline & & & 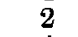 & & $\mathbf{M} \odot$ & 1 & 1 & & \\
\hline Or* & 10 & $\mathbf{R}$ & 4 & $\because$ & $\mathbf{M} \odot_{1}$ & 1 & 2 & & \\
\hline $\mathrm{Fe}^{*}$. & 5 & $\ldots$ & 7 & $d$ & $\mathbf{M} \odot_{l}$ & 2 & 2 & & 360 \\
\hline Fet ..... & 4 & & 6 & $\ldots$ & & 2 & 2 & & \\
\hline $\begin{array}{l}\mathrm{Fe} \text {. } \\
\mathbf{Y} \mathrm{t}\end{array}$ & 15 & 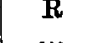 & 15 & $\cdots$ & $M \odot$ & 11 & 10 & $\begin{array}{l}360 \\
361\end{array}$ & \\
\hline $\mathrm{Fe}$. & 4 & & 4 & $\begin{array}{l}\cdots \\
\ldots\end{array}$ & $\odot^{\prime}$ & 1 & 15 & $3612 \cdot 237$ & $3612 \cdot 21$ \\
\hline $\mathrm{Ca}$ & $\cdots$ & $\cdots$ & 2 & d & $M \odot$ & 1 & 1 & 3617.939 & 3617.92 \\
\hline $\mathrm{Fe}\} .$. & 4 & & 3 & & $M \cup_{1}$ & 1 & & 5017.90У & \\
\hline Fe ......... & 20 & $\mathbf{R}$ & 20 & $\ldots$ & $\mathbf{M} \odot_{1}$ & 11 & 10 & 361 & 36 \\
\hline Yt ..... & 3 & $\ldots$ & 1 & $\ldots$ & & 1 & 1 & 362 & \\
\hline $\mathrm{Fe}$ & 4 & $\cdots$ & 4 & $\ldots$ & $\mathbf{M} \odot$ & 2 & 2 & 362 & \\
\hline & 4 & $\ldots$ & 4 & $\ldots$ & $\mathbf{M} \bigodot_{1}$ & 2 & 3 & 362 & 362 \\
\hline $\mathrm{Fe}$. & 4 & ... & 4 & ... & $\mathbf{M} \odot^{\prime \prime}$ & 1 & 14 & $3623 \cdot 338$ & 362 \\
\hline & 2 & $\cdots$ & 3 & ... & $\bigodot^{\prime i}$ & & 10 & & 362 \\
\hline & $\mathbf{3}$ & 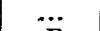 & 2 & ... & $M \cap$ & 1 & 1 & 53 & \\
\hline Fe... & 20 & $\mathbf{R}$ & 20 & $\dddot{m}$ & $\mathbf{M} \bigodot_{1}$ & 11 & 10 & 36 & 36 \\
\hline Yt\| & 5 & & & d & & 1 & 1 & & \\
\hline $\mathrm{Ti}{ }^{2} \ldots \ldots \ldots$. & 10 & $\mathbf{R}$ & 3 & $\cdots$ & $\mathbf{M} \odot^{\prime}$ & 3 & 1 & 15 & 36 \\
\hline$F_{\theta}$. & 5 & & 5 & $\ldots$ & $\mathbf{M} \bigodot_{1}$ & 1 & 1 & & 36 \\
\hline $\mathrm{Pb} \mathbf{T}$ & 50 & $\mathbf{R}$ & 1 & $\ldots$ & & 4 & ... & 362 & \\
\hline $\mathrm{Cr}+\ldots .$. & $2\}$ & $\cdots$ & 5 & $\cdots$ & $\mathbf{M} \odot{ }^{\prime \prime}$ & 1 & 14 & $3640 \cdot 545$ & $3640 \cdot 536$ \\
\hline 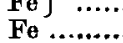 & 10 & $\mathbf{R}$ & 10 & $\ldots$ & $\mathbf{M} \odot$ & 10 & 11 & 3647.995 & 36 \\
\hline Co ..... & 5 & I & 3 & $\ldots$ & 0 & $\ldots$ & 5 & & 36 \\
\hline $\mathbf{T i}$ & 10 & R & 4 & $\ldots$ & $\mathbf{M} \odot^{\prime}$ & 2 & 7 & $|3653.639|$ & $3653 \cdot 63$ \\
\hline $\operatorname{Mn}\} \ldots$. & 2] & $\cdots$ & 2 & $\cdots$ & $\odot^{\prime \prime}$ & $\ldots$ & 7 & ....... & $3658 \cdot 688$ \\
\hline $\begin{array}{l}\mathrm{Fe} \\
\mathrm{Fe}\end{array}$ & 5 & & 3 & $\ldots$ & $\rho^{\prime \prime}$ &.. & 13 & & 36 \\
\hline Fo. & 8 & $\boldsymbol{R}$ & 8 & $\cdots$ & $\mathbf{M} \odot$ & 8 & 7 & 3680.064 & 3680.064 \\
\hline & 9 & & & & & & & & \\
\hline $\mathrm{Fe}$ & 1 & ... & 6 & $\cdots$ & $\odot^{\prime \prime}$ & 1 & 13 & $|3683 \cdot 209|$ & 3683.202 \\
\hline $\begin{array}{l}\nabla \mathrm{a}\} \\
\mathrm{Pb}\end{array}$ & 4] & R & 1 & & & 5 & & & \\
\hline & 00 & & 6 & $\cdots$ & $\odot^{\prime \prime}$ & 1 & 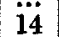 & & \\
\hline Fe.. & 10 & $\mathbf{R}$ & 8 & $\ldots$ & $\mathbf{M} \odot$ & 8 & 6 & & \\
\hline & & & & 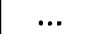 & M॰ & 1 & 1 & & \\
\hline $\mathbf{F}$ & 5 & 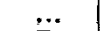 & 5 & ... & & 1 & 11 & & \\
\hline $\mathrm{Foth}$ & 7 & $\mathbf{R}$ & & & $\mathbf{M} \odot$ & 7 & 5 & & \\
\hline & 5 & & & $\boldsymbol{m}$ & & 1 & 11 & & 370 \\
\hline & 10 & $\mathbf{R}$ & 10 & $\ldots$ & $\mathbf{M} \bigodot_{1}$ & 6 & 4 & & \\
\hline $\mathbf{Y t}$ & 10 & $\cdots$ & 3 & $\cdots$ & & 1 & 1 & $|3710 \cdot 442|$ & 3710.43 \\
\hline
\end{tabular}

* In the solar speotrum these belong to a group of several lines. Of the three most prominent, the middle line is $\mathrm{Cr}$, with possibly a weak line on its red edge; and the red one is a close double, the violet component of the double being Fe. (J.)

+ The solar line is a group of four lines. The third from the violet side is the brightest and is Fe.

$\ddagger$ Metal measured was $\mathrm{Fe}$.

\& There is a faint line on the red side.

i. Red component of double.

In the solar spectrum this is the red component of a double, the otber being cobalt.

** The metallic line measured was $\mathrm{Fe}$.

t十 Violot component of a double. 
New Table of Standard Wave-lengths.

\begin{tabular}{|c|c|c|c|c|c|c|c|c|c|}
\hline \multirow{2}{*}{ Elements. } & \multicolumn{2}{|c|}{ In Arc. } & \multicolumn{2}{|c|}{ In Sur. } & \multirow{2}{*}{$\begin{array}{c}\text { Kind } \\
\text { of } \\
\text { Stand. } \\
\text { ard. }\end{array}$} & \multicolumn{2}{|c|}{ Wetght. } & \multirow{2}{*}{$\begin{array}{c}\text { Wave- } \\
\text { length } \\
\text { in Arc. }\end{array}$} & \multirow{2}{*}{$\begin{array}{l}\text { Ware- } \\
\text { length } \\
\text { in Sun. }\end{array}$} \\
\hline & $\begin{array}{l}\text { Inten- }- \\
\text { sity. }\end{array}$ & $\begin{array}{l}\text { Appear } \\
\text { ance. }\end{array}$ & $\begin{array}{l}\text { Inten- } \\
\text { sity. }\end{array}$ & $\begin{array}{l}\text { Appear- } \\
\text { ance. }\end{array}$ & & $\begin{array}{c}\text { In } \\
\text { Are. }\end{array}$ & $\begin{array}{l}\text { In } \\
\text { Sun. }\end{array}$ & & \\
\hline $\begin{array}{l}\mathrm{Fe} . \\
\mathrm{Fe} .\end{array}$ & 41 & R. & 7 & & "2 & 11 & $\begin{array}{l}12 \\
10\end{array}$ & $\begin{array}{l}3716 \cdot 601 \\
3720 \cdot 082\end{array}$ & \\
\hline$\underset{\mathrm{Ne}-\mathrm{Ti}}{\mathrm{Ni}}\}^{*}$ & 4 & & 10 & d & $\mathbf{M} \odot$ & $\begin{array}{r}11 \\
7\end{array}$ & 5 & 3722712 & $3722 \cdot 69$ \\
\hline $\mathrm{Fe}$ & 6 & $\mathbf{R}$ & 7 & $\cdots$ & $\mathbf{M}$ & 5 & 3 & $3727 \cdot 768$ & $37 \%$ \\
\hline $\mathrm{Fe}$ & 5 & & 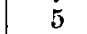 & $\cdots$ & $\odot^{\prime \prime}$ & 1 & 15 & & \\
\hline $\mathrm{Fe}$. & 6 & $R$ & 7 & $\ldots$ & $M \odot$ & 5 & 3 & $37^{\circ}$ & \\
\hline $\mathrm{Fe}$. & 40 & $\mathbf{R}$ & 50 & $\ldots$ & $\mathbf{M} \odot$ & 8 & 7 & 3735.012 & 375 \\
\hline $\left.\begin{array}{l}\mathbf{N i} \\
\mathbf{M n}\end{array}\right\} \ldots .$. & $\begin{array}{l}6 \\
2\end{array}$ & $\cdots$ & $3\}$ & $\mathrm{d}$ & $\odot_{1}$ & ... & 2 & ....... & 3736.96 \\
\hline $\mathrm{Ca}$............. & 4 & $\mathbf{R}$ & & ... & $\mathbf{M} \odot_{1}$ & 2 & 3 & 3737081 & 373 \\
\hline $\mathrm{Fe} .$. & 25 & $\mathbf{R}$ & 30 & $\ldots$ & $\mathbf{M} \odot$ & $\overline{7}$ & 8 & 3737.280 & 3737.2 \\
\hline $\mathrm{Ti} j$ & 3 & $\cdots$ & 2] & & & & & & \\
\hline $\mathrm{Fe}\}^{*} .$. & 5 & $\ldots$ & $6\}$ & $\mathrm{t}$ & $\mathbf{M} \odot$ & 4 & 2 & 3743506 & 374350 \\
\hline Cr J $\quad$ Fet & 3 & & $2]$ & & & & & & \\
\hline $\begin{array}{ll}\text { Fet } & \ldots . \\
\text { Fe }\end{array}$ & $\begin{array}{r}10 \\
7\end{array}$ & $\begin{array}{l}\mathbf{R} \\
\mathbf{R}\end{array}$ & $\begin{array}{r}10 \\
7\end{array}$ & $\cdots$ & $\frac{M}{M}$ & $\begin{array}{l}8 \\
6\end{array}$ & $\begin{array}{l}6 \\
5\end{array}$ & & 374 \\
\hline $\mathrm{Fe}]^{*} \quad .$. & 31 & & 7 & d & $\Omega^{\prime}$ & 1 & 9 & 08 & $3747 \cdot 09$ \\
\hline$\stackrel{?}{\mathrm{~F} \theta} \int^{\prime} \quad \cdot$ & $\stackrel{?}{10}$ & $\mathbf{R}$ & 10 & $\mathrm{u}$ & 8 & 7 & & 410 & $20 / 7800$ \\
\hline $\mathrm{Fe} . . . . . .$. & 20 & $\mathbf{R}$ & 20 & $\cdots$ & $\mathbf{M} \odot$ & 7 & $\begin{array}{l}8 \\
8\end{array}$ & & \\
\hline 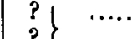 & ... & $\cdots$ & 2 & d & $\odot$ &.. & 12 & ....... & $3754 \cdot 66$ \\
\hline $\mathrm{Fe} \quad \ldots . . . .$. & 2 & $\cdots$ & 2 & & $\odot^{\prime}$ & 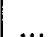 & 12 & & \\
\hline $\mathrm{Fe}$ & 15 & $\dddot{R}$ & 15 & & $\mathbf{M} \odot$ & $\dddot{8}$ & 7 & 37 & \\
\hline $\mathrm{Fe}$. & 9 & $\mathrm{R}$ & 10 & & $\mathbf{M}$ & 9 & 8 & & \\
\hline $\mathrm{Fe}$ & 7 & R & 0 & & $\mathbf{M} \odot$ & 9 & 8 & 3767.342 & \\
\hline $\mathrm{Fe} . . . . . .$. & 3 & $\cdots$ & 4 & $d$ ? & $\odot$ & $\ldots$ & 12 & & \\
\hline$Y_{t} ? \ldots$. & 6 & & 3 & $\ldots$ & $\mathbf{M} \odot$ & 1 & 1 & 377 & \\
\hline $\operatorname{Th} \ldots . . . . .$. & 40 & $\mathbf{R}$ & 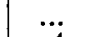 & $\cdots$ & M & 1 & & $37 \%$ & \\
\hline & & $\cdots$ & 4 & $\ldots$ & $\odot^{\prime}$ & $\cdots$ & 15 & & \\
\hline & 2 & & 3 & & $\odot^{\prime}$ & $\ldots$ & 15 & & \\
\hline & 10 & $\mathbf{R}$ & 6 & & $\odot$ & $\ldots$ & 15 & & \\
\hline & 7 & R & 8 & & $\mathbf{M} \odot$ & 3 & 3 & 3788.029 & \\
\hline $\mathrm{Fe}-\mathrm{Cr}$.. & 11 & .. & 3 & .. & $\odot$ & $\ldots$ & 15 & & \\
\hline $\mathrm{Fe}_{+}^{+} \ldots . . . .$. & 8 & $\because$ & 8 & $\ldots$ & $\mathbf{M}$ & 3 & 4 & $3795 \cdot 148$ & \\
\hline $\mathrm{Fe} \ldots \ldots \ldots$ & 7 & . & 7 & ... & $\odot$ & $\ldots$ & 2 & & \\
\hline $\mathrm{Fe}$ & 8 & .. & 8 & & $\odot$ & & 2 & & \\
\hline ...... & 2 & $\cdots$ & 3 & . & $\bigodot^{\prime \prime}$ & $\ldots$ & 15 & & \\
\hline$F \theta-D i \quad .$. & $4 ?$ & & 6 & & & $\ldots$ & 15 & & \\
\hline $\mathrm{F}$ & 20 & $\mathbf{R}$ & 20 & & $\mathbf{M} \odot$ & 4 & 3 & 381 & \\
\hline & 30 & $\mathbf{R}$ & 30 & & $\mathbf{M} \odot$ & 4 & 4 & $3820 \cdot 566$ & \\
\hline & 5 & & 6 & & $\odot^{\prime}$ & $\cdots$ & 10 & & 38 \\
\hline $\operatorname{Mn}(\mathrm{Cr})$. & 51 & $\mathbf{R}$ & & & $\odot^{\prime}$ & $\ldots$ & 10 & & 38 \\
\hline & 20 & $\mathbf{R}$ & 20 & & $\mathbf{M} \odot$ & 4 & 4 & 3826.024 & \\
\hline & 8 & $\mathbf{R}$ & 8 & .. & & 1 & 1 & 3827.973 & \\
\hline & 20 & $\mathbf{R}$ & 0 & & & $\cdots$ & 2 & & \\
\hline & 30 & $\mathbf{R}$ & 10 & $\ldots$ & $\mathbf{M}$ & $\cdots$ & 2 & $\ldots . .$. & 38324 \\
\hline & $\cdots$ & $\cdots$ & 4 & $\ldots$ & $\odot$ & $\cdots$ & 8 & & \\
\hline Mg. & 40 & $R_{1}$ & 90 & d & $\mathrm{M} \odot_{1}$ & 1 & 1 & $3836 \cdot 638$ & 38 \\
\hline Mg. & & & 20 & $\ldots$ & & ... & 2 & ...... & 38 \\
\hline
\end{tabular}

* The metallic line measured was Fe.

+ Violet portion of broad solar double is composed of three lines, the red line is Fe and the middle one Co. (J.) I There is a Va line towards the violet. $\S$ Central line of symmetrical group in carbon band. 


\begin{tabular}{|c|c|c|c|c|c|c|c|c|c|}
\hline \multirow{2}{*}{ Elements, } & \multicolumn{2}{|c|}{ In ARC. } & \multicolumn{2}{|c|}{ In Sun. } & \multirow{2}{*}{$\begin{array}{c}\text { Kind } \\
\text { of } \\
\text { Stand- } \\
\text { ard. }\end{array}$} & \multicolumn{2}{|c|}{ WEIGHT. } & \multirow{2}{*}{$\begin{array}{l}\text { Wave- } \\
\text { length } \\
\text { in Arc. }\end{array}$} & \multirow{2}{*}{$\begin{array}{l}\text { Wave- } \\
\text { length } \\
\text { in Sun. }\end{array}$} \\
\hline & $\begin{array}{c}\text { Inten- } \\
\text { sity. }\end{array}$ & $\begin{array}{l}\text { Appear- } \\
\text { ance. }\end{array}$ & $\begin{array}{c}\text { Inten- } \\
\text { sity. }\end{array}$ & $\begin{array}{l}\text { Appear- } \\
\text { ancé. }\end{array}$ & & $\begin{array}{c}\text { In } \\
\text { Arc. }\end{array}$ & $\mid \begin{array}{c}\text { In } \\
\text { Sun. }\end{array}$ & & \\
\hline & 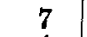 & $\mathbf{R}$ & 7 & & $\mathbf{M}$ & & 2 & $3840 \cdot 589$ & \\
\hline & 4 & & & & $\odot$ & & 8 & 1 & \\
\hline & 6 & & 7 & $\cdots$ & $\mathbf{M}$ & 1 & 2 & & 38 \\
\hline & $\begin{array}{c}10 \\
\ldots\end{array}$ & $\mathbf{R}$ & $\begin{array}{r}10 \\
3\end{array}$ & 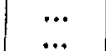 & $\mathbf{M} \odot_{I}$ & 2 & $\begin{array}{l}3 \\
8\end{array}$ & 3860.050 & \\
\hline & $\cdots$ & $\ldots$ & 4 & $\cdots$ & $\mathbf{M}$ & $\dddot{4}$ & 4 & $3871 \cdot 527$ & $3871 \cdot 5$ \\
\hline & $\cdots$ & $\cdots$ & 3 & $\cdots$ & $\odot$ & $\ldots$ & 15 & ....... & $3875 \cdot 2$ \\
\hline of & $\because$ & $\cdots$ & 7 & $\cdots$ & $\mathbf{M} \odot_{1}$ & 5 & 3 & 3883.479 & $3883 \cdot 4$ \\
\hline & $\cdots$ & $\ldots$ & $\because$ & $\ldots$ & $\mathbf{M} \bigodot_{\mathbf{l}}$ & $\ldots$ & 8 & 3883.523 & 388 \\
\hline & $\ldots$ & $\ldots$ & 1 & $\cdots$ & $\odot$ & & 12 & & 388 \\
\hline $\mathbf{F}$ & 15 & $\mathbf{R}$ & 9 & $\cdots$ & $\mathbf{M} \odot_{1}$ & 7 & 6 & $3886 \cdot 421$ & 388 \\
\hline & 3 & & 4 & $\cdots$ & $\bigodot^{\prime}$ & & 12 & & 38 \\
\hline & 10 & $\mathbf{R}$ & 10 & " & $\mathbf{M}$ & 4 & 4 & $3905 \cdot 670 \mid$ & 390 \\
\hline$\stackrel{\mathrm{F}}{\mathrm{T}}$ & $\begin{array}{l}3 \\
6\end{array}$ & ... & $\begin{array}{l}3 \\
4\end{array}$ & $\cdots$ & $\Theta^{\prime}$ & 1 & 12 & $3916 \cdot 886$ & 39 \\
\hline & $\begin{array}{l}0 \\
1\end{array}$ & . & 4 & .* & $\odot^{\prime}$ & $\cdots$ & 15 & ...... & 392 \\
\hline & $2\}$ & ... & 4 & ... & $\odot^{\prime}$ & $\cdots$ & 15 & ...... & 392 \\
\hline & 3 & $\cdots$ & 4 & ... & $\odot$ & ... & 13 & $\cdots$ & $3925 \cdot 79$ \\
\hline $\left.\begin{array}{c}\mathrm{Fe} \\
?\end{array}\right\}$ & $\begin{array}{l}5 \\
?\end{array}$ & $\ldots$ & $\left.\begin{array}{l}4 \\
4\end{array}\right\}$ & d & $\odot^{\prime}$ & $\cdots$ & 12 & ...... & $3926 \cdot 12$ \\
\hline $\mathrm{Fe} \ldots . . .$. & 10 & $\dddot{R}$ & 8 & & M & 1 & 3 & $9928 \cdot 0<0$ & 392 \\
\hline K Ca T & 75 & $\mathbf{R}$ & 300 & & $\mathbf{M}$ & 6 & 5 & $3933 \cdot 809$ & \\
\hline Fe & 3 & & 4 & & $\bigodot^{\prime}$ & $\ldots$ & 8 & & 3937 \\
\hline F-C & 44 & $\cdots$ & 5 & $\cdots$ & $\odot$ & 1 & 15 & $3941 \cdot 034$ & 3941.02 \\
\hline & $\frac{?}{5}$ & $\ldots$ & $\begin{array}{l}2 \\
4\end{array}$ & $\mathrm{~d}$ & $\Theta$ & $\ldots$ & 15 & ...... & $3942 \cdot 55$ \\
\hline & 20 & $\dddot{R}$ & 10 & ... & $\mathbf{M} \odot_{1}$ & 7 & 7 & $944 \cdot 165$ & 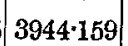 \\
\hline & 4 & .. & & $\ldots$ & & i & 2 & 3949.070 & \\
\hline & 4 & & 4 & & $\odot^{\prime \prime}$ & & 15 & ....... & 395 \\
\hline & 10 & $\cdots$ & 2 & $\cdots$ & $\odot$ & $\ldots$ & 13 & ....... & \\
\hline $\mathbf{F}$ & 2 & 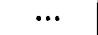 & & & $\odot^{\prime}$ & & 13 & & \\
\hline $\mathrm{Fe}-\mathrm{Ca}$. & 56 & $\cdots$ & 6 & $\cdots$ & $\odot^{\prime}$ & 1 & 2 & $3957 \cdot 228$ & 3957 \\
\hline & 3 & R & & $\cdots$ & $\rho^{\prime \prime}$ & $\dddot{7}$ & $\begin{array}{ll}11 \\
8\end{array}$ & 80 & 960 \\
\hline $\mathrm{Ca}, \ldots$ & 70 & $\mathbf{R}$ & 200 & $\cdots$ & $\mathbf{M}^{\mathbf{M} \cup_{1}}$ & 7 & 5 & $3968 \cdot 617$ & $\begin{array}{l}396167 \\
3968.62\end{array}$ \\
\hline & & & $\ldots$ & & & $\ldots$ & & $3970-05$ & \\
\hline Fesูg ... & 5 & & 4 & & 5 & $\ldots$ & 11 & & 3971 \\
\hline $\mathrm{Ca} \| \ldots$ & 5 & & 3 & d & & 1 & 2 & 3973.881 & \\
\hline $\mathbf{F}$ & $\boldsymbol{J}$ & & 4 & $\cdots$ & & $\cdots$ & 15 & 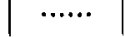 & $397 \%$ \\
\hline
\end{tabular}

* One of the lines in the carbon band.

+ Second head of carbon band.

First line of first head of carbon band.

Edge of first head of carbon band.

|| 087 apart.

The solar line is doubly reversed and spread out into broad shading for 6.000 or 7.000 on either side. In each case the second reversal is slightly eccentric with respect to the other, being displaced towards the red. (J.)

** Components 085 apart.

t† Red component of a triple.

t Value determined by Dr. Ames.

Red component of a double.

Red component of double, the violet component boing $\mathrm{Fe}_{\mathrm{e}}$. There is also a $\mathrm{Ni}$ line close to violet side. 
New Table of Standard Wave-lengths.

\begin{tabular}{|c|c|c|c|c|c|c|c|c|c|}
\hline \multirow{2}{*}{ Elements. } & \multicolumn{2}{|c|}{ IN Arc. } & \multicolumn{2}{|c|}{ IN SUn. } & \multirow{2}{*}{$\begin{array}{l}\text { Kind } \\
\text { of } \\
\text { Stand- } \\
\text { ard. }\end{array}$} & \multicolumn{2}{|c|}{ WhiaIrT. } & \multirow{2}{*}{$\begin{array}{l}\text { Ware- } \\
\text { length } \\
\text { in Arc. }\end{array}$} & \multirow{2}{*}{$\begin{array}{l}\text { Wave- } \\
\text { length } \\
\text { in Sun. }\end{array}$} \\
\hline & $\begin{array}{l}\text { Inten- } \\
\text { sity. }\end{array}$ & $\begin{array}{c}\text { Appear- } \\
\text { ance. }\end{array}$ & $\begin{array}{l}\text { Inten- } \\
\text { sity. }\end{array}$ & $\begin{array}{l}\text { Appear- } \\
\text { ance. }\end{array}$ & & $\begin{array}{l}\text { In } \\
\text { Arc. }\end{array}$ & $\begin{array}{l}\text { In } \\
\text { Sun. }\end{array}$ & & \\
\hline $\mathrm{Fe}-$ & 69 & ... & 4 & ... & $\odot$ & $\ldots$ & 14 & $\ldots \ldots$ & 3981 \\
\hline $\begin{array}{l}\mathrm{Cr} \\
\mathbf{F e}\end{array}$ & $3\}$ & $\cdots$ & 6 & d & $\odot$ & $\cdots$ & 9 & ...... & 3984 \\
\hline $\left.\begin{array}{c}? \\
\mathrm{Mn}\end{array}\right\}$ & $?$ & $\ldots$ & $\left.\begin{array}{l}7 \\
1\end{array}\right\}$ & d & (๐) & ... & 9 & $\cdots$ & 986 \\
\hline $\begin{array}{l}\mathrm{Mn} \ldots \\
\mathrm{Co} \ldots\end{array}$ & $\left.\begin{array}{l}4 \\
2\end{array}\right\}$ & $\cdots$ & 6 & $\mathrm{t}$ & $\odot$ & $\ldots$ & 4 & ....... & $3987 \cdot 2$ \\
\hline $\mathrm{Ce}-\mathrm{Fe}-\mathrm{Ti}$. & 412 & $\ldots$ & 3 & $\ldots$ & $\odot$ & $\ldots$ & 9 & ....... & 00 \\
\hline$\cdots$ & 10 & $\cdots$ & $\begin{array}{r}10 \\
3\end{array}$ & $\cdots$ & $\stackrel{\odot}{\odot}$ & $\cdots$ & $\begin{array}{l}3 \\
7\end{array}$ & …... & 4016.5 \\
\hline & $\ddot{2}$ & 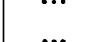 & 4 & $\cdots$ & $\odot^{\prime \prime}$ & $\cdots$ & 10 & $\ldots \ldots$ & $4029 \cdot 7$ \\
\hline $\mathrm{Z}_{\mathrm{M}}$ & $2\}$ & 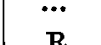 & 7 & $\ldots$ & $M$ & 3 & 4 & $4030 \cdot 919$ & 4030.9 \\
\hline $\mathbf{M n}$ & 25 & $\mathbf{R}$ & 6 & $\because$ & $\mathbf{M}$ & 3 & 4 & & \\
\hline Mnq & 20 & $\mathbf{R}$ & 5 & $\ldots$ & M & 3 & 4 & 4034 & 40 \\
\hline $\mathrm{Mn} \boldsymbol{q}$ & 7 & & 3 & $\cdots$ & M. & 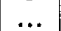 &.. & 4035 & 408 \\
\hline $\mathbf{K} .$. & 50 & R & 1 & $\ldots$ & M & 2 & 2 & 4044 & \\
\hline ....... & 20 & $\vec{R}$ & 20 & $\cdots$ & $\mathrm{M} \odot_{1}$ & 7 & 7 & 4045 & 4045 \\
\hline K... & 40 & $\mathbf{R}$ & $1 ?$ & $\ldots$ & $\mathbf{M}$ & 2 & $\ldots$ & $4047 \cdot 373$ & \\
\hline $\begin{array}{l}\mathbf{Z} \mathbf{r} \\
\mathbf{M n}_{\mathbf{n}}\end{array}$ & 1) & & 6 & & & & 13 & & $4048 \cdot 8$ \\
\hline & 8 & $\cdots$ & 0 & & & $\cdot$ & & $\cdots$ & \\
\hline $\mathrm{Mn} \ldots . .$. & 8 & $\ldots$ & 5 & $\ldots$ & $\odot^{\prime \prime}$ & $\ldots$ & 13 & ....... & 405 \\
\hline ....... & 5 & & & ... & $\odot$ & . & 8 & & \\
\hline ....... & 15 & $\mathbf{R}$ & 15 & ... & $\mathbf{M} \odot_{1}$ & 7 & 7 & 4063755 & 406 \\
\hline ....... & 10 & ... & 10 & .. & $\mathbf{M}$ & 7 & 9 & 4071.903 & \\
\hline $\mathrm{Fe}$ & 4 & $\ldots$ & 4 & $\ldots$ & $\odot^{\prime}$ & $\ldots$ & 14 & & 407 \\
\hline Sr.. & 50 & $\mathbf{R}$ & 8 & $\ldots$ & $\mathbf{M} \odot_{1}$ & 5 & 6 & $4077 \cdot 876$ & 4077 \\
\hline $\mathrm{F}$ & $?$ & $\ldots$ & 2 & $\mathrm{~d}$ & $\odot$ & $\ldots$ & 7 & ....... & 4083.76 \\
\hline$\frac{1}{H}$ & 5 & $\cdots$ & $\begin{array}{l}45 \\
2\end{array}$ & & 8 & $\cdots$ & 7 & 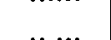 & \\
\hline $\mathrm{F}$ & 2 & $\cdots$ & 2 & $\cdots$ & $\odot^{\circ}$ & ... & 8 & $\because \cdots \cdots$ & 408 \\
\hline ... & 3 & & 6 & $\cdots$ & " & . & 10 & 年 & 4103 \\
\hline & $1\}$ & $\cdots$ & 0 & & & $\cdots$ & & ….. & \\
\hline ..... & 5 & $\ldots$ & 5 & d? & $\Theta^{\prime \prime}$ & ... & 12 & ....... & 410 \\
\hline & 3 &. & 4 & $\cdots$ & 1 & $\ldots$ & 14 & ....... & 411 \\
\hline$+\dagger .$. & 1 & $\dddot{\mathrm{R}}$ & 1 & .. & $\odot$ & 1 & 12 & $4121 \cdot 476$ & $4121 \cdot 48$ \\
\hline Cr... & 31 & $\ldots$ & 3 & $\cdots$ & $0^{\prime}$ & cot & 13 &. & \\
\hline & 4 & $\cdots$ & 3 & $\cdots$ & ○" & … & 17 & & 4157.9 \\
\hline$\overline{\mathrm{C}}$ & $\ldots$ & $\ldots$ & & $\ldots$ & $\mathbf{M}$ & $\ldots$ & 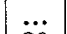 & $4158 \cdot 2$ & \\
\hline & 4 & & 3 & & $\odot$ & $\ldots$ & 20 & & \\
\hline & $\ldots$ & $\ldots$ & 1 & $\ldots$ & $\mathbf{M} \mathbf{0}$ & 5 & 6 & $4197 \cdot 25$ & \\
\hline
\end{tabular}

* Components about 060 apart.

+ Hazy line shaded to red. Shading is due to a Mn line on red side.

t Triple line. Central line is brightest.

$\$$ Seven or eight lines. The brightest and most of the others are due to $\mathrm{Fe}$.

II Violet component of double, being itself double or reversed in Sun. The other component is weak.

T Red component of double, being itself double or reversed in Sun. The other component is weak.

** An unequal double, violet component being much the weaker.

$+\dagger$ Cobalt line was measured.

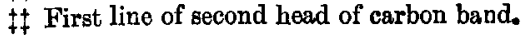


Prof. H. A. Rowland on a

\begin{tabular}{|c|c|c|c|c|c|c|c|c|c|}
\hline \multirow{2}{*}{ Elements. } & \multicolumn{2}{|c|}{ In Arc. } & \multicolumn{2}{|c|}{ IN Sun. } & \multirow{2}{*}{$\begin{array}{l}\text { Kind } \\
\text { of } \\
\text { Stand- } \\
\text { ard. }\end{array}$} & \multicolumn{2}{|c|}{ Weiglit. } & \multirow{2}{*}{$\begin{array}{l}\text { Wave- } \\
\text { length } \\
\text { in Arc. }\end{array}$} & \multirow{2}{*}{$\begin{array}{l}\text { Wave- } \\
\text { length } \\
\text { in Sun. }\end{array}$} \\
\hline & $\begin{array}{l}\text { Inten- } \\
\text { sity. }\end{array}$ & $\begin{array}{c}\text { Appear- } \\
\text { ance. }\end{array}$ & $\begin{array}{l}\text { Inten- } \\
\text { sity. }\end{array}$ & $\begin{array}{l}\text { Appear } \\
\text { ance. }\end{array}$ & & In & Sun & & \\
\hline $\left.\begin{array}{l}\mathrm{Zr} \\
\mathrm{Fr}_{\theta}\end{array}\right\}:$ & $\left.\begin{array}{l}2 \\
5\end{array}\right\}$ & $\cdots$ & 5 & $\cdots$ & $\odot^{\prime \prime}$ & 2 & 22 & $4199 \cdot 257$ & $4199 \cdot 26$ \\
\hline $\mathrm{Fe}$ & 8 & & 5 & $\cdots$ & $\odot^{\prime}$ & 2 & 4 & $4202 \cdot 187$ & $4202 \cdot 188$ \\
\hline $\mathrm{Fe}$ & 2 & 2 & $\cdots$ & $\dddot{d}$ & $\odot$ & $\begin{array}{l}\ldots \\
\ldots\end{array}$ & $\begin{array}{r}\tilde{2} \\
18\end{array}$ & S- & $\begin{array}{l}4215 \cdot 616 \\
4215 \cdot 667\end{array}$ \\
\hline $\mathrm{Sr}$ & 40 & $\mathbf{R}$ & 4 & ... & $\mathbf{M} \odot$ & 6 & 3 & 4215688 & 4215.687 \\
\hline $\mathrm{O}^{*}$ & $\ldots$ & $\cdots$ & 1 & $\cdots$ & $\mathbf{M} \odot$ & 4 & 2 & $4216 \cdot 133$ & $4216 \cdot 137$ \\
\hline Fe $\ldots \ldots$ & 2 & $P$ & 4 & $\cdots$ & $\stackrel{\odot}{\prime \prime}$ & 1 & $\begin{array}{l}22 \\
10\end{array}$ & $\left|\begin{array}{l}4222 \cdot 396 \\
4226 \cdot 898\end{array}\right|$ & $\begin{array}{l}4222 \cdot 381 \\
4296 \cdot 89\end{array}$ \\
\hline $\mathrm{g}_{\mathrm{Fe}} \ldots \ldots$ & $\begin{array}{r}50 \\
4\end{array}$ & $\mathbf{R}$ & $\begin{array}{r}10 \\
5\end{array}$ & $\cdots$ & $\begin{array}{l}\mathrm{M} \odot \\
\odot^{\prime}\end{array}$ & $\begin{array}{l}y \\
I\end{array}$ & $\begin{array}{r}10 \\
1\end{array}$ & $\mid \begin{array}{l}4220090 \\
4250 \cdot 300\end{array}$ & $\begin{array}{l}4226 \cdot 892 \\
4250 \cdot 290\end{array}$ \\
\hline Fe $\ldots$ & $\begin{array}{l}4 \\
5\end{array}$ & $\cdots$ & 7 & $\ldots$ & $\odot^{\prime}$ & $\tilde{4}$ & 3 & 4250.949 & 4250.956 \\
\hline $\mathrm{Cr}$ & 20 & $\mathbf{R}$ & 7 & .. & $\mathbf{M} \odot^{\prime \prime}$ & 2 & 15 & $4254 \cdot 494$ & $4254-502$ \\
\hline $\mathrm{Fe}$ & 6 & $\mathbf{R}$ & 7 & $\ldots$ & $\odot$ & 4 & 3 & $4260 \cdot 647$ & $4260 \cdot 638$ \\
\hline$\stackrel{?}{\mathrm{~F}}$ & ? & .. & $\left.\begin{array}{l}1 \\
2\end{array}\right\}$ & d & $\odot$ & $\cdots$ & 12 & ....... & $4267 \cdot 958$ \\
\hline $\mathrm{Fe}$ & 10 & $\dddot{R}$ & 8 & $\ldots$ & $\mathbf{M} \odot$ & 8 & 9 & $4271 \cdot 920$ & $4271 \cdot 924$ \\
\hline $\mathrm{Cr}$ & 15 & $\mathbf{R}$ & 5 & $\ldots$ & $\mathbf{M} \odot$ & 1 & 2 & 427 & \\
\hline $\mathrm{Ca} \quad \ldots . .$. & 5 & $\mathbf{R}$ & 3 & $\cdots$. & $\mathrm{M} \odot$ & 2 & 4 & 428 & 428 \\
\hline $\mathrm{Ca}$ & 4 & $\mathbf{R}$ & 3 & ..... & $\mathbf{M} \odot$ & 3 & 5 & 4288 & .428 \\
\hline ..... & 10 & $\mathbf{R}$ & 4 & $\ldots$ & $\mathbf{M} \odot$ & 2 & 2 & 4289.884 & 4289.881 \\
\hline ..... & $\cdots$ & 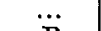 & 4 & d & $\odot^{\prime}$ & $\cdots$ & 14 & & 429 \\
\hline $\mathrm{Ca} \quad \ldots . .$. & 3 & $\mathbf{R}$ & 2 & $\cdots$ & $\mathbf{M} \odot$ & 3 & 5 & $4299 \cdot 153$ & $4299 \cdot 152$ \\
\hline $\mathrm{Ca} \quad \ldots . .$. & 6 & $\mathbf{R}$ & 4 & $\cdots$ & M & 5 & 7 & 430 & $4302.689 \mid$ \\
\hline $\mathrm{Sr}$ & 8 & $\dddot{\dddot{m}}$ & 2 & $\ldots$ & $\mathbf{M}$ & 1 & $\cdots$ & 430 & \\
\hline Ti & 10 & $\mathbf{R}$ & 4 & $\cdots$ & $\mathbf{M} \odot$ & 4 & 4 & 4306 & 4306.071 \\
\hline$G^{\mathrm{Ca}}$ & 4 & $\mathbf{R}$ & 2 & 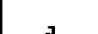 & $\odot$ & 3 & 3 & 4307.906 & 4307.904 \\
\hline $\mathrm{G}_{\mathrm{Fe}}$ & & $\mathbf{R}$ & 5 & d & $\odot$ & 8 & $\begin{array}{r}3 \\
10\end{array}$ & 4308.072 & $\begin{array}{l}4308 \cdot 034 \\
4308 \cdot 071\end{array}$ \\
\hline $\mathrm{Ca}$ & 4 & $\mathrm{R}$ & 3 & $\cdots$ & $\mathbf{M} \odot "$ & 3 & 16 & 431 & 4318.818 \\
\hline $\mathrm{fFe}$ & 10 & $\mathbf{R}$ & 8 & $\ldots$ & $\mathbf{M} \odot{ }^{\prime}$ & 8 & 15 & 4325.932 & 4325.940 \\
\hline $\mathrm{Cr}$ & 2 & $\cdots$ & 1 & d? & $\odot$ & $\ldots$ & 11 & ....... & $4343 \cdot 387$ \\
\hline $\begin{array}{l}\mathrm{Fe} \\
\mathrm{Fe}\end{array}$ & 4 & $\cdots$ & 3 & ... $>$ & $\odot \prime \prime$ & 1 & 17 & 4352.908 & $4352 \cdot 903$ \\
\hline $\begin{array}{l}\mathrm{Fe} \\
\mathrm{Ni}\end{array}$ & $\begin{array}{l}4 \\
3\end{array}$ & .. & $\begin{array}{l}3 \\
1 \\
\end{array}$ & $\cdots$ & $0^{3}$ & 1 & 18 & 4302908 & 4302903 \\
\hline $\mathrm{Cr}$ & 4 & ... & 3 & $t$ & $\odot$ & $\ldots$ & 10 & ... & 4359.778 \\
\hline $\mathrm{Zr}$ & 5 & $\cdots$ & 1 & & & & & & \\
\hline $\mathbf{F e}$ & 4 & $\cdots$ & 5 & $\cdots$ & $\odot^{\prime \prime}$ & 1 & 14 & $4369 \cdot 948$ & 4369.94 \\
\hline $\begin{array}{r}\mathrm{Fe} \\
\mathrm{d} \mathrm{Fe}\end{array}$ & 5 & & 5 & $\cdots$ & $\ominus^{\prime \prime}$ & 1 & 17 & $\mid 4376 \cdot 108$ & $4376 \cdot 103$ \\
\hline $\begin{array}{c}\mathrm{dFe} \\
\mathrm{Fe}\rceil \ldots \ldots\end{array}$ & 15 & $\mathbf{R}$ & 10 & $\cdots$ & $\mathbf{M} \odot{ }^{\prime}$ & 10 & 11 & 4383721 & $4383 \cdot 721 \mid$ \\
\hline $\left.\begin{array}{l}\mathrm{Fe} \\
\mathrm{Ti}\end{array}\right\}^{\dagger} \ldots$ & $\begin{array}{l}2 \\
1\end{array}$ & $\cdots$ & $\begin{array}{l}3 \\
1\end{array}$ & $\cdots$ & $\odot$ & $\cdots$ & 14 & $\ldots \ldots$ & $4391 \cdot 149$ \\
\hline Fe & 10 & $\mathbf{R}$ & 8 & $\cdots$ & $\mathbf{M} \odot$ & 10 & 11 & $4404 \cdot 9 \cdot 28$ & 4404.927 \\
\hline $\mathrm{Va}$ & 9 & $\mathbf{R}$ & $?$ & d & $\odot$ & $\ldots$ & 19 & ...... & $4407 \cdot 850$ \\
\hline $\begin{array}{l}\text { Fe } \\
\text { Cd }\end{array}$ & b & $\begin{array}{l}\cdots \\
\ldots\end{array}$ & $\begin{array}{l}3 \\
6\end{array}$ & - & $\mathbf{M}$ & 3 & & 4413 & \\
\hline ....... & 1 & $\mathrm{R}$ & 4 & $\ldots$ & $\mathbf{M} \odot$ & 9 & 7 & 441 & $441:$ \\
\hline .. & 5 & $\mathbf{R}$ & 4 & $\ldots$ & $\mathbf{M} \odot "$ & 5 & 7 & 442 & \\
\hline$\cdots . . .1$ & 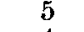 & $\mathbf{R}$ & 4 & $\ldots$ & M & 5 & 5 & 443 & $4435 \cdot 132$ \\
\hline $\mathrm{Ca} \quad \ldots . .$. & 4 & $\mathbf{R}$ & & ... & $\mathbf{M} \odot "$ & 5 & 6 & 443 & $4435^{\circ}$ \\
\hline Fet $\ldots . .$. & 8 & & ( & $\cdots$ & & 2 & 18 & $4447 \cdot 912$ & 444 \\
\hline $\mathrm{Ca} \S$ & 6 & $\mathbf{R}$ & 6 & $\cdots$ & $M \odot_{1}$ & 6 & 6 & $4454 \cdot 949$ & | 4454:95 \\
\hline
\end{tabular}

* First line in first head of carbon band.

+ Unequal double, components being about -050 apart.

t There is a faint side line towards the red.

$\$$ There is a faint line close to violet side. 
New Table of Standard Wave-lengths.

\begin{tabular}{|c|c|c|c|c|c|c|c|c|c|}
\hline \multirow{2}{*}{ Ilements. } & \multicolumn{2}{|c|}{ IN ARc. } & \multicolumn{2}{|c|}{ In Sun. } & \multirow{2}{*}{$\begin{array}{c}\text { Kind } \\
\text { of } \\
\text { Stand- } \\
\text { ard. }\end{array}$} & \multicolumn{2}{|c|}{ WeIGIIT. } & \multirow{2}{*}{$\begin{array}{l}\text { Wave- } \\
\text { length } \\
\text { in Arc. }\end{array}$} & \multirow{2}{*}{$\begin{array}{l}\text { Wave- } \\
\text { length } \\
\text { in Sun. }\end{array}$} \\
\hline & $\begin{array}{c}\text { Inten- } \\
\text { sity. }\end{array}$ & $\begin{array}{c}\text { Appear- } \\
\text { ance. }\end{array}$ & $\begin{array}{c}\text { Inten. } \\
\text { sity. }\end{array}$ & $\begin{array}{l}\text { Appear- } \\
\text { ance. }\end{array}$ & & $\begin{array}{l}\text { In } \\
\text { Arc. }\end{array}$ & $\begin{array}{l}\text { In } \\
\text { Sun. }\end{array}$ & & \\
\hline $\mathrm{O}$ & 3 & $\mathrm{R}$ & 2 & & $M$ & 5 & 3 & 55 & \\
\hline & 1 & $\cdots$ & & & $\mathbf{M} \odot$ & 2 & $\stackrel{3}{2}$ & & \\
\hline & & $\ldots$ & 0 & $\cdots$ & $\odot^{\prime \prime}$ & 1 & $1 \overline{8}$ & 756 & \\
\hline & $\begin{array}{l}5 \\
2\end{array}$ & $\cdots$ & 4 & $\cdots$ & $\odot$ & $\ldots$ & 14 & ....... & 4497.04 \\
\hline $\begin{array}{l}2 \mathrm{r} \\
\mathbf{M}_{n}\end{array}$ & & 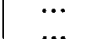 & 1 & & $0^{\prime \prime}$ &.. & 8 & & \\
\hline & $\cdots$ & ?.. & 1 & d & $\odot^{\prime}$ & $\ddot{\ldots}$ & 7 & …... & \\
\hline & 6 & $\ldots$ & 5 & $\cdots$ & $\odot^{\prime}$ & $\cdots$ & 18 & & $4501^{\circ}$ \\
\hline & $\ldots$ & $\ldots$ & & $\ldots$ & & $\ldots$ & & $4502 \cdot 6$ & \\
\hline & $\ldots$ & $\ldots$ & 4 & $\ldots$ & $\odot^{\prime \prime}$ & $\ldots$ & 17 & 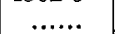 & 4508 \\
\hline & $\ldots$ & $\ldots$ & ... & $\ldots$ & M & 4 & $\cdots$ & $4511 \cdot 474$ & \\
\hline & & & & $\ldots$ & & 3 & & & \\
\hline & 70 & $\mathbf{R}$ & 7 & $\ldots$ & $\mathbf{M} \odot "$ & 6 & 8 & 455 & \\
\hline & 4 & $\ldots$ & 6 & $\ldots$ & $\odot^{\prime \prime}$ & & 13 & & 45 \\
\hline & 3 & $\ldots$ & 5 & . & $0^{\prime \prime}$ & 1 & 14 & $457 \overline{1} \cdot 281$ & \\
\hline & 5 & & 6 & $\ldots$ & $\bigodot^{\prime \prime}$ & $\ldots$ & 14 & & 45 \\
\hline & 31 & $\ddot{N}$ & 4 & $\ldots$ & $\odot^{\prime \prime}$ & $\ddot{1}$ & 14 & 4578.807 & \\
\hline$\ldots$ & $\cdots$ & $\ldots$ & 4 & $\ldots$ & $\odot^{\prime \prime}$ & $\ldots$ & 14 & $\ldots \ldots$ & \\
\hline & $\ldots$ & $\ldots$ & 4 & & $\odot^{\prime \prime}$ & 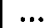 & 15 & & \\
\hline & 2 & & 4 & $\ldots$ & $\odot^{\prime \prime}$ & $\ldots$ & 20 & & 4602 \\
\hline $\mathbf{L}$ & 50 & $\dddot{R}$ & $\ldots$ & $\ldots$ & $\mathbf{M}$ & $\ddot{1}$ & $\ldots$ & $4602: 25$ & \\
\hline C & & & & . & & & & & \\
\hline Sr & 50 & $\mathbf{R}$ & 2 & $\cdots$ & $\mathbf{M} \odot \odot^{\prime}$ & 5 & 4 & $4607 \cdot 506$ & 4607.50 \\
\hline$\stackrel{?}{\mathrm{Fe}}\}^{\S} \ldots$ & $\begin{array}{l}? \\
4\end{array}$ & $\ldots$ & $\begin{array}{l}2 \\
6\end{array}$ & d & $\odot_{1}$ & $\ldots$ & 11 & ....... & $4611 \cdot 4$ \\
\hline & 4 & & 5 & & $\Omega^{\prime}$ & & & & \\
\hline & 5 & $\cdots$ & 5 & .. & $\odot^{\prime}$ & $\cdots$ & 13 & $\cdots \cdots$ & 462 \\
\hline $\mathbf{F}$ & 3 & & 4 & & $\odot^{\prime}$ & $\ldots$ & 14 & ....... & \\
\hline ... & - & $\ldots$ & 4 & $\ldots$ & $\odot^{\prime}$ & $\ldots$ & 14 & $\ldots \ldots$ & \\
\hline & 2 & & 4 & & $\odot^{\prime \prime}$ & $\ldots$ & 17 & & \\
\hline & 6 & $\mathbf{R}$ & 3 & $\ldots$ & $\mathbf{M} \odot$ & 1 & 1 & $4648 \cdot 833$ & 4648.8 \\
\hline & $\because$ & $\cdots$ & 2 & $d$ & $\odot^{\prime}$ & $\ldots$ & 11 & $\ldots$ & 4668.30 \\
\hline $\mathrm{Cd}$ & & & 4 ? & ... & $\mathbf{M}$ & 3 & 3 & 4678.339 & \\
\hline & 3 & & $\ldots$ & $\ldots$ & $0^{\prime}$ & & 12 & & \\
\hline $\mathbf{Z}$ & & & 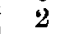 & & $\mathbf{M}$ & 1 & & $4680 \cdot 319$ & \\
\hline & 2 & & & & $\odot^{\prime \prime}$ & $\ldots$ & 13 & & \\
\hline $\mathbf{N}$ & 4 & • & 4 & $\cdots$ & $\odot^{\prime \prime}$ & $\ldots$ & 12 & $\ldots$ & \\
\hline & & & 4 & $\cdots$ & $\odot^{\prime}$ & $\ldots$ & 14 & $\ldots . .$. & $4690 \cdot 3$ \\
\hline$\| \ldots$ & 3 & & 2 & d & $\odot$ & $\ldots$ & 11 & $\ldots \ldots$ & 4691 \\
\hline & 3 & & 9 & 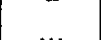 & " & 1 & 11 & $4703 \cdot 249$ & \\
\hline & $\begin{array}{l}5 \\
3\end{array}$ & $\ldots$ & 3 & $\cdots$ & $\odot^{\prime \prime}$ & $\lambda$ & 13 & & \\
\hline$\cdots \cdots$ & 9 & $\dddot{\mathbf{R}}$ & 6 & $\ddot{d} ?$ & $\mathbf{M} \odot$ & $\dddot{1}$ & 1 & $\dddot{4}: 59$ & \\
\hline $\mathrm{Zn}$ & 4 & $\ldots$ & 4 & $\ldots$ & $\mathbf{M} \odot "$ & 2 & 2 & $4722 \cdot 339$ & $4722 \cdot 34$ \\
\hline n & $\frac{2}{7}$ & $\cdots$ & $4\}$ & $d$ & $\odot$ & $\ldots$ & 11 & ....... & $4727 \cdot 62$ \\
\hline & & & & & & & & & \\
\hline
\end{tabular}

* Red component of a double. Other line is $\mathrm{Mn}$.

† First line in first head of blue carbon band.

\$ Strong line with fine line very close to violet side and another farther to violet.

$\$$ Besides the double live measured there is another fine line near the red side.

11 The $\mathrm{Mg}$ line is of the nature of a band, shaded toward the red. It coincides with the solar line when there is very little material in the arc. (R.)

T Solar line is shaded towards the violet, probably owing to a close side line.

* Much the same in character as the red lithium line. (J.) 


\begin{tabular}{|c|c|c|c|c|c|c|c|c|c|}
\hline \multirow{2}{*}{ Flements. } & \multicolumn{2}{|c|}{ Iv ÁRC. } & \multicolumn{2}{|c|}{ IN Sun. } & \multirow{2}{*}{$\begin{array}{l}\text { Kind } \\
\text { of } \\
\text { Stand- } \\
\text { ard. }\end{array}$} & \multicolumn{2}{|c|}{ WeIGHT. } & \multirow{2}{*}{$\begin{array}{l}\text { Ware- } \\
\text { length } \\
\text { in Arc. }\end{array}$} & \multirow{2}{*}{$\begin{array}{l}\text { Wave- } \\
\text { length } \\
\text { in Sun. }\end{array}$} \\
\hline & $\begin{array}{l}\text { Inten- } \\
\text { sity. }\end{array}$ & $\begin{array}{c}\text { Appear- } \\
\text { ance. }\end{array}$ & $\begin{array}{l}\text { Inten- } \\
\text { sity. }\end{array}$ & $\begin{array}{l}\text { Appear- } \\
\text { ance. }\end{array}$ & & $\begin{array}{c}\text { In } \\
\text { Arc. }\end{array}$ & $\begin{array}{c}\text { In } \\
\text { Sun. }\end{array}$ & & \\
\hline & 15 & & 6 & & $\odot^{\prime \prime}$ & ... & 11 & & \\
\hline & 10 & & & & $\mathbf{M} \odot \odot^{\prime \prime}$ & 1 & 1 & 4783.607 & \\
\hline C & 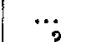 & “. & $?$ & $\cdots$ & & 3 & $\therefore$ & $4800 \cdot 097$ & \\
\hline$\stackrel{?}{\mathrm{~T}}$ & $?$ & $\because$ & $\begin{array}{l}1 \\
4\end{array}$ & d & $\odot_{1}$ & $\cdots$ & 3 & $\cdots \cdots$ & $4805 \cdot 2$ \\
\hline $\mathbf{Z n}_{\mathbf{n}}$ & & $\cdots$ & 3 & $\ldots$ & $M \odot^{\prime \prime}$ & 1 & 1 & $4810 \cdot 725$ & 481 \\
\hline M & 10 & ... & 6 & ... & $\mathbf{M} \odot^{\prime \prime}$ & 1 & 12 & 482 & \\
\hline & 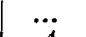 & $\cdots$ & 4 & $\cdots$ & $\odot^{\prime \prime}$ & $\ldots$ & 11 & ....... & 482 \\
\hline & 4 & $\cdots$ & $\mathbf{5}$ & $\ldots$ & $\bigodot^{\prime \prime}$ & $\ldots$ & 14 & $\cdots$ & \\
\hline $\mathrm{H}$ & & $\cdots$ & 15 & $\ldots$ & $\bigodot^{\prime}$ & $\ldots$ & 5 & ....... & \\
\hline $\mathrm{Fe}$. & 7 & $\ldots$ & 7 & $\ldots$ & $\odot^{\prime \prime}$ & $\cdots$ & 11 & ...... & \\
\hline $\mathrm{Ti}^{*}$. & 4 & $\cdots$ & 2 & d & $\Theta^{\prime}$ & $\cdots$ & 11 & $\ldots \ldots$ & 90 \\
\hline & 9) & $\cdots$ & $2\}$ & & $\odot^{\prime}$ & $\cdots$ & 11 & ...... & 490 \\
\hline & $\left.\begin{array}{l}2 \\
5\end{array}\right\}$ & $\cdots$ & 6 & $\mathrm{~d}$ ? & $\odot^{\prime \prime}$ & ... & 14 & $\ldots \ldots$ & $4903 \cdot 48$ \\
\hline & & $\cdots$ & & & M & 1 & $\cdots$ & 4905.634 & \\
\hline & 6 & & 7 & & $\odot^{\prime \prime}$ & & 4 & & 491 \\
\hline & 9 & & 9 & & $\mathbf{M} \odot^{\prime \prime}$ & 1 & 7 & $4920 \cdot 676$ & 492 \\
\hline & 2 & $\ldots$ & 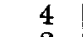 & & $\odot^{\prime \prime}$ & $\ldots$ & 13 & $\ldots \ldots$. & 492 \\
\hline & 3 & 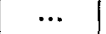 & 2 & & $\odot^{\prime \prime}$ & $\ldots$ & 12 & & 492 \\
\hline B & 60 & .. & 7 & d? & $\widetilde{\mathbf{M}} \odot$ & 1 & 10 & $4934 \cdot 237$ & 493 \\
\hline & $\cdots$ & & 6 & $\cdots$ & $\mathbf{M} \odot_{1}$ & $\ldots$ & 3 & $\ldots \ldots$ & 4957 \\
\hline F & $\cdots$ & .. & 8 & $\cdots$ & $\mathbf{M} \odot_{1}$ & $\ldots$ & 3 & 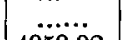 & $4957 \cdot 78$ \\
\hline $\mathbf{N}$ & 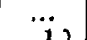 & $\cdots$ & $\cdots$ & $\cdots$ & $\cdots$ & $\cdots$ & ... & 4959.02 & \\
\hline & $\left.\begin{array}{l}1 \\
3\end{array}\right\}$ & - & 3 & *. & $\odot^{\prime \prime}$ & $\cdots$ & 10 & ....... & $4973 \cdot 27$ \\
\hline & 3 & .. & $1\}$ & d? & $\odot^{\prime \prime}$ & & 8 & & 497878 \\
\hline $\begin{array}{l}\mathrm{Fe} \\
\mathrm{Ni}\end{array}$ & $\begin{array}{l}3 \\
5\end{array}$ & & 3 & & & $\cdots$ & & $\cdots$ & \\
\hline & ? & . & $\left.\begin{array}{l}0 \\
1\end{array}\right\}$ & & $\odot$ & $\ldots$ & 5 & $\ldots .$. & $4980 \cdot 362$ \\
\hline Ti.. & 10 & $\cdots$ & 4 & .. & $\odot$ & 1 & 10 & $4981 \cdot 893$ & 498 \\
\hline & 3 & & & & & $\ldots$ & 7 & & 499 \\
\hline$\| \ldots$ & 1010 & R N & 4 & ... & $\mathbf{M} \odot$ & $\cdots$ & 8 & 999 & 4999 \\
\hline & 3 & .. & & $\cdots$ & & 5 & & $5005 \cdot 634$ & \\
\hline $\mathrm{F}$ & 3 & $\cdots$ & 6 & . & $\bigodot_{\odot}^{\prime \prime}$ & $\cdots$ & 10 & $\ldots .$. & 5005 \\
\hline & 10 & $\dddot{\boldsymbol{R}}$ & 0 & 舟 & & $\cdots$ & & …... & \\
\hline & 3 & $\ldots$ & , & $d$ & $\odot^{\prime \prime}$ & $\ldots$ & 10 & ...... & $5007 \cdot 431$ \\
\hline & $\cdots$ & $\cdots$ & $\ldots$ & ... & & $\cdots$ & $\cdots$ & 5007.05 & \\
\hline$t+$ & & .. & & & M & 3 & 10 & $5007 \cdot 473$ & \\
\hline $\left.\begin{array}{c}(\mathrm{Ni}) \mathrm{Ti} \\
\mathrm{Ti}\end{array}\right\}$ & $\begin{array}{r}? 10 \\
5\end{array}$ & $\ldots$ & $\left.\begin{array}{l}3 \\
4\end{array}\right\}$ & $d$ & $M \odot^{\prime}$ & $\cdots$ & 10 & $5014 \cdot 412$ & $5014 \cdot 422$ \\
\hline & 7 & $\ldots$ & s. & ... & $\odot^{\prime}$ & $\ldots$ & 8 & $\ldots \ldots$ & 5020.210 \\
\hline & 6 & $\cdots$ & $3)$ & $d$ & $\odot^{\prime}$ & $\cdots$ & 8 & $\cdots$ & $5036 \cdot 115$ \\
\hline $\begin{array}{l}\mathrm{Nij} \\
\mathrm{Ca}\end{array}$ & 3 & $\mathbf{N}$ & 2 & $\ldots$ & $\mathrm{M}$ & 2 & 1 & $5041 \cdot 867$ & $5041 \cdot 795$ \\
\hline & & & & & & & & & \\
\hline
\end{tabular}

* A Ba line comes between these and does not coincide with either.

+ Shaded, and has a faint line towards the red.

A very difficult double with a fine line towards the violet. (J.)

There is a faint line towards the red.

Ti line was measured.

There is a faint side line towards the violet.

* Valnes determined by J. E. Keeler from bis measurements at the Lick

Observatory using the values of the $\mathrm{Pb}, \mathrm{Fe}$, and $\mathrm{Mg}$ lines given in this table.

t+ Commencement of the head of $\mathbf{M g}$ band. 


\begin{tabular}{|c|c|c|c|c|c|c|c|c|c|}
\hline \multirow{2}{*}{ Elements. } & \multicolumn{2}{|c|}{ IN ARC. } & \multicolumn{2}{|c|}{ Ir Sur. } & \multirow{2}{*}{$\begin{array}{c}\text { Kind } \\
\text { of } \\
\text { Stand- } \\
\text { ard. }\end{array}$} & \multicolumn{2}{|c|}{ W niantr. } & \multirow{2}{*}{$\begin{array}{l}\text { Ware- } \\
\text { length } \\
\text { in Arc. }\end{array}$} & \multirow{2}{*}{$\begin{array}{l}\text { Ware- } \\
\text { length } \\
\text { in Sun. }\end{array}$} \\
\hline & $\begin{array}{l}\text { Inten- } \\
\text { sity. }\end{array}$ & $\begin{array}{c}\text { Appear- } \\
\text { ance. }\end{array}$ & $\begin{array}{l}\text { Inten- } \\
\text { sity. }\end{array}$ & $\begin{array}{c}\text { Appear- } \\
\text { ance. }\end{array}$ & & \begin{tabular}{|} 
In \\
Arc.
\end{tabular} & $\begin{array}{l}\text { In } \\
\text { Sun. }\end{array}$ & & \\
\hline $\mathrm{Fe}$ & 5 & $\therefore$ & 5 & & $\odot^{\prime \prime}$ & 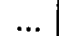 & 12 & ... & \\
\hline & 2 & $\ldots$ & 2 & $\ldots$ & $\odot^{\prime}$ & $\ldots$ & 15 & & \\
\hline $\mathrm{Ti}$ & 10 & $\ldots$ & $\overline{3}$ & $\ldots$ & $\bigodot^{\prime}$ & $\ldots$ & 12 & ........ & \\
\hline $\mathrm{Fe}$. & 4 & $\ldots$ & 4 & $\cdots$ & $\bigodot^{\prime \prime}$ & $\ldots$ & 14 & ....... & \\
\hline & 4 & $\ldots$ & 9 & $\therefore$ & $\odot^{\prime \prime}$ & $\ldots$ & 9 & & 508 \\
\hline Od & & $\ldots$ & $?$ & $\cdots$ & M & 1 & $\cdots$ & 5086.001 & \\
\hline$F$ & 3 & $\cdots$ & 2 & $\cdots$ & $\odot^{\prime \prime}$ & $\ldots$ & 9 & $\ldots \ldots$ & 509 \\
\hline $\begin{array}{l}\mathrm{F} \\
\mathrm{F}\end{array}$ & $\begin{array}{l}3 \\
3 \\
3\end{array}$ & $\cdots$ & & $\cdots$ & $\odot_{i}$ & $\cdots$ & 7 & $\cdots$ & \\
\hline $\mathrm{Fe}$ & 3 & $\cdots$ & 2 & $\cdots$ & $\stackrel{\odot}{\odot}^{\prime \prime}$ & $\ldots$ & 12 & $\begin{array}{l}\cdots \\
\cdots\end{array}$ & \\
\hline & ? & $\ldots$ & $2\}$ & $\mathrm{d}$ & $\odot^{\prime}$ & 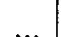 & 11 & & \\
\hline $\begin{array}{l}\mathrm{Fe}\} \\
\mathrm{Ni}\end{array}$ & 4 & $\cdots$ & 35 & & $\odot$ & & & ...... & 5110.57 \\
\hline $\begin{array}{l}\mathrm{Ni} \\
\mathrm{Ni}\end{array}$ & 5 & $\cdots$ & 2 & $\cdots$ & $\odot^{\prime \prime}$ & $\ldots$ & 9 & ...... & 5115.558 \\
\hline$\ldots$ & $\begin{array}{l}2 \\
8\end{array}$ & $\cdots$ & $\left.\begin{array}{l}1 \\
3\end{array}\right\}$ & $d$ & $\odot_{1}$ & $\ldots$ & 9 & ....... & $5121 \cdot 79$ \\
\hline $\mathrm{Co}$ & 3 & $\cdots$ & 2 & $\cdots$ & $\odot^{\prime \prime}$ & $\ldots$ & 9 & .. & 5126 \\
\hline $\begin{array}{l}\mathrm{Fe} \\
\mathrm{Fe}\end{array}$ & 4 & $\cdots$ & 4 & $\ldots$ & $\odot^{\prime \prime}$ & $\cdots$ & 9 & $\cdots \cdots$ & $5127 \cdot 53$ \\
\hline & $\left.\begin{array}{l}1 \\
?\end{array}\right\}$ & $\cdots$ & 6 & d & $\odot^{\prime \prime}$ & $\ldots$ & 12 & $\ldots . .$. & $5133 \cdot 871$ \\
\hline $\mathrm{Fe}_{\theta}$ & 4 & ... & 67 & $\dddot{d}$ & $\odot$ & $\cdots$ & 4 & ....... & \\
\hline $\mathrm{Fe}$ & 5 & & 6 & $\ldots$ & $\odot$ & $\cdots$ & $\begin{array}{l} \pm \\
4\end{array}$ & ........ & \\
\hline $\mathrm{Fe}$ & 2 & & 3 & 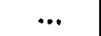 & $\odot^{\prime \prime}$ & $\cdots$ & 5 & ... & \\
\hline $\mathrm{Ni}$ & 5 & $\cdots$ & 2 & $\dddot{d}$ & $\odot_{1}$ & .. & 1 & ...... & 514 \\
\hline $\mathrm{Fe}$ & $\boldsymbol{J}$ & .. & 4) & $\begin{array}{l}\text { a } \\
. .\end{array}$ & $\begin{array}{l}\ominus_{1} \\
\bigodot_{1}\end{array}$ & $\cdots$ & 5 & $\cdots$ & \\
\hline $\mathrm{Ni}$ & 5 & 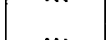 & 3 & $\cdots$ & $\begin{array}{l}\odot_{1} \\
\odot^{\prime \prime}\end{array}$ & $\cdots$ & 2 & $\cdots$ & 1514 \\
\hline & ? & $\cdots$ & 3) & $\cdots$ & $\odot^{\prime \prime}$ & $\cdots$ & 10 & ...... & 5146664 \\
\hline $\left.\begin{array}{l}\mathrm{Fo} \\
\mathbf{M n}\end{array}\right\} \neq .$. & $\begin{array}{l}4 \\
2\end{array}$ & $\begin{array}{l}\ldots \\
\ldots\end{array}$ & $\left.\begin{array}{l}3 \\
1\end{array}\right\}$ & d & $\odot$ & $\cdots$ & 9 & $\ldots$ & $5151 \cdot 026$ \\
\hline Ti? Co? & & $\ldots$ & & ... & $\odot^{\prime \prime}$ & .. & 10 & ... & \\
\hline & 6 & $\cdots$ & & $\cdots$ & $\bigodot^{\prime \prime}$ & & 10 & ..... & \\
\hline F ?. & & $\cdots$ & & ... & & $\ldots$ & 11 & ....... & \\
\hline $\mathrm{Fe}$. & 4 & $\cdots$ & 4 & $\cdots$ & $\odot^{\prime \prime}$ & $\dddot{m}$ & 13 & & \\
\hline $\mathrm{C}$ & $\dddot{0}$ & ... & & $\cdots$ & $M$ & 2 & 1 & $5165 \cdot 241$ & \\
\hline $\mathrm{Fe}$ & 2 & $\mathbf{R}$ & 2 & $\cdots$ & $\odot^{\prime \prime}$ & $\dddot{a}$ & 10 & 00 & \\
\hline $\mathrm{Mg}$ & 20 & $\mathbf{K}$ & & $\dddot{d}$ & $\begin{array}{l}\mathbf{M} \odot_{1} \\
\odot\end{array}$ & 2 & $\begin{array}{l}3 \\
7\end{array}$ & $67 \cdot 488$ & 5167 \\
\hline $\mathrm{Fe}$ & 6 & $\ldots$ & 6 & $\ldots$ & $\mathbf{M} \odot_{1}$ & 2 & 3 & $5167 \cdot 664$ & \\
\hline $\mathrm{F}$ & 3 & $\ldots$ & 4 & & $\odot^{\prime \prime}$ & $\cdots$ & 3 & & 5169 \\
\hline f & & & & d & & & 0 & 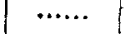 & \\
\hline $\begin{array}{ll}\mathrm{Fe} & \\
\mathrm{Fe}\end{array}$ & $\begin{array}{l}3 \\
5\end{array}$ & $\begin{array}{l}\cdots \\
\ldots\end{array}$ & $\mathbf{4}$ & $\cdots$ & $\bigodot^{\prime \prime \prime}$ & $\cdots$ & $\begin{array}{r}3 \\
11\end{array}$ & 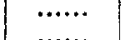 & \\
\hline $\mathrm{b}_{2} \mathrm{Mg} \ldots$ & 35 & $\mathbb{R}$ & 10 & ". & $\mathbf{M} \odot \odot^{\prime \prime}$ & 2 & 9 & $5172 \cdot 866$ & $5172 \cdot 871$ \\
\hline
\end{tabular}

* The fine line near to violet side belongs to $\mathrm{Ni}$ ?

+ There is a Cr line near to red side.

$\$$ The $\mathrm{Mn}$ line is a faint side line towards the red from the $\mathrm{Fe}_{\mathrm{e}}$ line.

\$ Measurements in the arc spectrum were on the first line of the first bead of the green carbon band; measurements in the solar spectrum were probably on the brightest of a group of faint lines near the head of carbon band (J.). Much of the band can be seen on my map of the solar speetrum extending to the left (R.).

I| Components about $0 \cdot 180$ apart on photographie plates. (J.)

- Components about 0.138 apart as measured by Rowland in solar spectrum and 0.150 apart as measured on photographic plates. (J.)

Phil. Mag. S. 5. Vol. 36. No. 218. July 1893. F F 


\begin{tabular}{|c|c|c|c|c|c|c|c|c|c|}
\hline \multirow{2}{*}{ Elements. } & \multicolumn{2}{|c|}{ In ARc. } & \multicolumn{2}{|c|}{ Is Sun. } & \multirow{2}{*}{$\begin{array}{l}\text { Kind } \\
\text { of } \\
\text { Stand- } \\
\text { ard. }\end{array}$} & \multicolumn{2}{|c|}{ Whight. } & \multirow{2}{*}{$\begin{array}{l}\text { Wave- } \\
\text { length } \\
\text { in Arc. }\end{array}$} & \multirow{2}{*}{$\begin{array}{l}\text { Wave- } \\
\text { length } \\
\text { in Sun. }\end{array}$} \\
\hline & $\begin{array}{c}\text { Inten- } \\
\text { sity. }\end{array}$ & $\begin{array}{c}\text { Appear- } \\
\text { ance. }\end{array}$ & $\begin{array}{c}\text { Inten- } \\
\text { sity. }\end{array}$ & $\begin{array}{l}\text { Appear- } \\
\text { ance. }\end{array}$ & & $\begin{array}{c}\text { In } \\
\text { Arc. }\end{array}$ & $\underset{\text { Sun. }}{\operatorname{In}}$ & & \\
\hline Ti.. & 10 & $\mathbf{R}$ & 3 & $\cdots$ & $\odot^{\prime \prime}$ & ... & 11 & & 5173.912 \\
\hline $\mathrm{Mg}$ & 40 & $\mathbf{R}$ & 20 & $\ldots$ & $\overline{\mathbf{M}} \odot^{\prime \prime}$ & 2 & 11 & 5183791 & $5183 \cdot 792$ \\
\hline Ti & 2 & $\cdots$ & 4 & $\dddot{x}$ & $\odot^{n}$ & $\cdots$ & 3 & $\ldots \ldots$ & \begin{tabular}{|c|}
$5188 \cdot 863$ \\
5188.048
\end{tabular} \\
\hline $\mathrm{Ca}$ & 6 & $\ldots$ & 4 & $\begin{array}{l}\text { d } \\
\ldots\end{array}$ & ${\stackrel{\odot}{\mathbf{M}} \odot^{\prime \prime}}^{\prime \prime}$ & $\ddot{1}$ & 3 & $5189 \cdot 019$ & $\begin{array}{l}5188.948 \\
5189.020\end{array}$ \\
\hline Ti... & 8 & $\ldots$ & 3 & $\ldots$ & $\overline{\mathbf{M}} \odot^{\prime \prime}$ & 2 & 8 & $5193 \cdot 134$ & $5193 \cdot 139$ \\
\hline $\mathrm{Fo}$ & 3 & ... & 4 & $\cdots$ & $\odot^{\prime \prime}$ & $\cdots$ & 10 & $\ldots$. & $5198 \cdot 885$ \\
\hline$\left.\stackrel{?}{\mathrm{~F}_{\theta}}\right\}$ & $\begin{array}{l}? \\
4\end{array}$ & $\cdots$ & $\left.\begin{array}{l}2 \\
3\end{array}\right\}$ & d & $\odot^{\prime \prime}$ & ... & 11 & ....... & $5202 \cdot 483$ \\
\hline $\mathrm{Cr}$ & 8 & $\dddot{R}$ & 4 & $d$ & $\Omega^{\prime \prime}$ & & 10 & & $5004 \cdot 70$ \\
\hline $\mathrm{Fe}\}$ & 3 & & $3\}$ & $\mathbf{a}$ & $\odot$ & 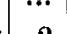 & 10 & …… & ontrog \\
\hline $\operatorname{Ti} \ldots . . . . . .$. & 10 & $\mathbf{R}$ & 3 & $\cdots$ & $\mathbf{M} \odot^{\prime \prime}$ & 2 & 12 & $5210 \cdot 549$ & $\begin{array}{l}5210 \cdot 556 \\
5215\end{array}$ \\
\hline $\mathrm{Fe} \quad \ldots . .$. & 3 & $\cdots$ & $\begin{array}{l}4 \\
4\end{array}$ & $\ldots$ & $\stackrel{\odot}{\odot}^{\prime \prime}$ & $\ldots$ & $\begin{array}{l}10 \\
10\end{array}$ & ........ & 5217.559 \\
\hline $\begin{array}{ll}\mathrm{Fe} & \ldots . . . \\
\mathrm{Fe} & \ldots . . .\end{array}$ & $\begin{array}{l}3 \\
2\end{array}$ & ... & 2 & ... & $\odot^{\prime \prime}$ & $\cdots$ & 10 & ....... & $5225 \cdot 690$ \\
\hline Fe $\ldots . . .$. & 4 & $\ldots$ & 4 & ... & $\odot^{\prime \prime}$ & $\ldots$ & 8 & ....... & $5230 \cdot 014$ \\
\hline Fe $\ldots$ & 7 & $\ldots$ & 8 & $\cdots$ & $\bigodot^{\prime \prime}$ & ... & 9 & ....... & $5233 \cdot 124$ \\
\hline $\mathrm{Fe} \ldots$. & 3 & $\ldots$ & 3 & $\cdots$ & $\odot^{\prime \prime}$ & $\cdots$ & 10 & ....... & 5242662 \\
\hline Fe $\ldots . . .$. & 2 & $\ldots$ & 2 & ... & $\bigodot^{\prime \prime}$ & $\cdots$ & 11 & ....... & 5250.391 \\
\hline Fe $\ldots . . .$. & 3 & $\ldots$ & 3 & $\ldots$ & $\odot^{\prime \prime}$ & $\cdots$ & 11 & ....... & $5250 \cdot 825 \mid$ \\
\hline $\mathrm{Fe}$ & 2 & $\cdots$ & 3 & $\cdots$ & $\Theta^{\prime \prime}$ & $\cdots$ & 12 & & $|5253 \cdot 649|$ \\
\hline $\mathrm{Ca}$ & 2 & $\cdots$ & 1 & $\ldots$ & $\overline{\mathbf{M}} \odot_{1}$ & 1 & 5 & 5260.556 & 5260.557 \\
\hline $\begin{array}{l}\mathrm{Ca} \\
\mathrm{Cr}\end{array}$ & $\left.\begin{array}{l}6 \\
2\end{array}\right\}$ & ... & 3 & $\cdots$ & $\odot$ & 1 & 12 & ....... & $5261-880$ \\
\hline ? & & $\cdots$ & $1)$ & 年 & $\mathbf{M} \odot_{1}$ & $\ldots$ & 1 & & $5262 \cdot 341$ \\
\hline$\dot{\mathrm{Ca}}$ & 6 & $\cdots$ & 2 & d & $\odot$ & 2 & 5 & $5262 \cdot 408$ & 5262.391 \\
\hline $\mathrm{Cr}$ & 4 & $\mathbf{R}$ & 3 & $\cdots$ & $\overbrace{}^{\prime \prime}$ & $\cdots$ & 3 & ...... & $|5264 \cdot 327|$ \\
\hline $\mathrm{Oa}$ & 6 & ... & 3 & .... & $\mathbf{M} \odot$ & 2 & 3 & $5264 \cdot 408$ & $|5264.395|$ \\
\hline $\mathrm{Ca}$ & 8 & $\cdots$ & 3 & $\cdots$ & $\odot$ & 1 & 2 & $|5265 \cdot 725|$ & 5265.727 \\
\hline (Nii) & & & 2 & & $\odot$ & $\cdots$ & 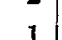 & ...... & $5285 \cdot 884$ \\
\hline $\mathrm{Cr}$ & 4 & $\mathbf{R}$ & 2 & $\cdots$ & 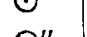 & $\cdots$ & 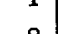 & …... & 0200004 \\
\hline $\mathrm{E}_{2}^{\mathrm{Fe}} \ldots$ & $\begin{array}{l}6 \\
8\end{array}$ & $\cdots$ & $\begin{array}{l}6 \\
8\end{array}$ & $\dddot{d} \ddot{q}$ & $\bigodot_{\odot}^{\prime \prime}$ & 1 & $\begin{array}{r}8 \\
16\end{array}$ & $\begin{array}{l}5266 \cdot 733 \\
5269 \cdot 714\end{array}$ & $\left|\begin{array}{l}5266 \cdot 729 \\
5269 \cdot 722\end{array}\right|$ \\
\hline $\mathrm{Ca}$ & 10 & $\cdots$ & 4) & & $\mathbf{M} \odot$ & 2 & 3 & $5270 \cdot 445$ & $5270 \cdot 448$ \\
\hline $\left.\mathrm{E}_{1}{ }_{\mathrm{Fe}}\right\}$ & 6 & & 4 & d & $\odot^{n}$ & $\cdots$ & 12 & $\ldots \ldots$ & $5270 \cdot 495$ \\
\hline $\begin{array}{l}\mathrm{Fe} \\
\mathrm{Fe}\end{array}$ & $\begin{array}{l}\mathbf{6} \\
\mathbf{3}\end{array}$ & $\cdots$ & 3 & $\cdots$ & $\Theta^{\prime \prime}$ & $\cdots$ & $\begin{array}{l}3 \\
6\end{array}$ & ….... & $\mid \begin{array}{l}0270.333 \\
5273 \cdot 344\end{array}$ \\
\hline & & & & d & $\odot^{\prime}$ & $\ldots$ & 5 & ........ & $5273 \cdot 443$ \\
\hline $\mathrm{Fe}$ & $\begin{array}{l}3 \\
?\end{array}$ & $\cdots$ & $\begin{array}{l}3] \\
21\end{array}$ & $\cdots$ & $\odot^{\prime \prime}$ & $\ldots$ & 8 & ...... & 5273.554 \\
\hline $\mathrm{Cr}\} \| \ldots$ & 5 & $\ldots$ & 2 & $t$ & $\odot^{\prime \prime}$ & $\ldots$ & 11 & ....... & $5276 \cdot 205$ \\
\hline Co & 3 & $\cdots$ & $1)$ & & & & & & \\
\hline & 4 & $\cdots$ & $\mathbf{0}$ & $\cdots$ & $\odot^{\prime \prime}$ & $\cdots$ & 11 & $\ldots \ldots$ & 5281.968 \\
\hline
\end{tabular}

* Components about $0-155$ apart on photographic plates. (J.)

+ Another set of measurements on photographic plates gives the components as 0.083 apart.

† Components about 0.088 apart on photographis plate. It is an exceedingly difficult double and it is possible that this doubleness of $\mathbf{E}_{2}$ is really a case of the reversal of line in the Sun. (J.)

$\S$ Components 0.077 apart as determined by another short series (R.); 0.130 on photographic plates (J.).

1/ Oomponents of double about 0075 apart on photographic plates. The fine side line to red is about $0 \cdot 110$ from the red component of double. (J.) 


\begin{tabular}{|c|c|c|c|c|c|c|c|c|c|}
\hline \multirow{2}{*}{ Elements. } & \multicolumn{2}{|c|}{ IN ARC. } & \multicolumn{2}{|c|}{ IN Sun. } & \multirow{2}{*}{$\begin{array}{l}\text { Kind } \\
\text { of } \\
\text { Stand } \\
\text { ard. }\end{array}$} & \multicolumn{2}{|c|}{ W EIGHT. } & \multirow{2}{*}{$\begin{array}{l}\text { Wave- } \\
\text { length } \\
\text { in Arc. }\end{array}$} & \multirow{2}{*}{$\begin{array}{l}\text { Wave- } \\
\text { length } \\
\text { in Sun. }\end{array}$} \\
\hline & $\begin{array}{r}\text { Inten. } \\
\text { sity. }\end{array}$ & $\begin{array}{l}\text { Appear } \\
\text { ance. }\end{array}$ & $\begin{array}{l}\text { Inten- } \\
\text { sity. }\end{array}$ & $\begin{array}{c}\text { Appear- } \\
\text { ance. }\end{array}$ & & $\begin{array}{l}\text { In } \\
\text { Arc. }\end{array}$ & $\begin{array}{l}\text { In } \\
\text { Sun. }\end{array}$ & & \\
\hline $\mathrm{Fe}$ & 5 & $\cdots$ & 6 & & $\odot^{\prime \prime}$ & & 11 & & 528 \\
\hline $\mathrm{Fe}$ & 2 & & 2 & & $\bigodot^{\prime \prime}$ & $\ldots$ & 12 & & \\
\hline $\mathrm{Cr}$ & 6 & $\mathbf{R}$ & 4 & & $\odot^{\prime \prime}$ & & 12 & .. & 529 \\
\hline $\mathrm{Cr}$. & 2 & $\cdots$ & 3 & & $\bigodot^{\prime \prime}$ & .... & 9 & ..... & 530 \\
\hline $\mathrm{Fe}$ & 3 & $\ldots$ & 4 & & $\odot^{\prime \prime}$ & $\cdots$ & 10 & & 530 \\
\hline $\mathrm{Fe}$ ? & $\ldots$ ? & & 4 & & $\odot$ & ... & 1 & ....... & 531 \\
\hline 1474 & & d & 6 & $d$ & $\odot$ & $\cdots$ & 7 & ........ & 53. \\
\hline Co? & 2 & $\ldots$ & 3 & ... & $\odot$ & $\ldots$ & i & & 531 \\
\hline Fo . & 9 & $\ldots$ & 8 & $\ldots$ & $\bigodot^{\prime \prime}$ & $\cdots$ & 8 & ....... & 582 \\
\hline $\mathrm{Fe} ? \ldots \ldots$ & 3 & $\cdots$ & 4 & - & $\odot^{\prime}$ & $\ldots$ & 9 & & 533 \\
\hline $\mathrm{Ca}$ & 7 & $\dddot{*}$ & 5 & ... & $\overline{\mathbf{M}} \odot$ & 1 & 4 & $5349 \cdot 599$ & $5349 \cdot 6$ \\
\hline $\operatorname{Tht} \ldots .$. & 75 & $\mathrm{t} ?$ & & $\ldots$ & $\mathbf{M}$ & 2 & $\cdots$ & $5350 \cdot 670$ & \\
\hline $\mathrm{Fe}-\mathrm{Ni}$ if & $3 \cdot 3$ & $\cdots$ & 4 & $\cdots$ & $\bigodot_{1}$ & $\ldots$ & 8 & ....... & 535 \\
\hline$?$ & $\cdots$ & $\cdots$ & 2 & $\ldots$ & $\odot$ & $\cdots$ & 7 & $\ldots .$. & 586 \\
\hline $\mathrm{Fe}(\mathrm{Go})\}_{?}^{\$}$ & 13 & $\cdots$ & $1\}$ & d & $\odot$ & $\ldots$ & 5 & ...... & 536 \\
\hline Fe & $?^{?}$ & $\cdots$ & 6 & & $\bigodot_{\bigodot^{\prime \prime}}^{\prime \prime}$ & $\cdots$ & 1 & .• & 5863 \\
\hline $\mathrm{Fe}$ & 4 & $\cdots$ & 6 & $\cdots$ & $\bigodot^{\prime \prime}$ & $\cdots$ & 8 & …... & $\begin{array}{l}5367.67 \\
5370 \cdot 16\end{array}$ \\
\hline $\mathrm{Ni}$ & 2 & $\ldots$ & 2 & d. & & & & & \\
\hline $\mathrm{Fe}-\mathrm{Cr}\}$ & 92 & ... & 7 & d & $\odot_{1}$ & $\cdots$ & 8 & $\cdots \cdots$ & $5371 \cdot 68$ \\
\hline Fe $\ldots \ldots$ & 2 & $\cdots$ & 3 & $\cdots$ & $\odot^{\prime \prime}$ & $\ldots$ & 9 & ....... & $5379 \cdot 7$ \\
\hline $\mathbf{F e}$ & 6 & $\cdots$ & 6 & $\ldots$ & $\Phi^{\prime \prime}$ & $\ldots$ & 11 & & \\
\hline $\mathrm{Fe} \quad \ldots . .$. & 3 & $\ldots$ & 4 & & $\bigodot^{\prime \prime}$ & $\ldots$ & 11 & ....... & 53 \\
\hline $\mathbf{F e}$ & 4 & י & 5 & $\cdots$ & $\bigodot^{\prime \prime}$ & $\ldots$ & 11 & & \\
\hline Fe & 7 & $\ldots$ & 7 & 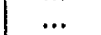 & $\bigodot^{\prime \prime}$ & 1 & 12 & 5397.319 & 5397 \\
\hline $\mathbf{F e}$ & 7 & & 7 & $\cdots$ & $\odot^{\prime \prime}$ & 1 & 14 & 5405.979 & $5405 \cdot 9$ \\
\hline Or & 10 & $\mathbf{R}$ & 5 & $\ldots$ & $\bigodot^{\prime \prime}$ & $\ldots$ & 7 & ........ & $5410 \cdot 00$ \\
\hline $\begin{array}{l}F_{e} \\
\nabla_{\mathbf{a}}\end{array}$ & $\left.\begin{array}{l}4 \\
4\end{array}\right\}$ & $\cdots$ & 6 & $\cdots$ & $\odot^{\prime \prime}$ & $\cdots$ & 12 & ...... & $5415 \cdot 42$ \\
\hline $\begin{array}{l}\overline{V a} \\
\mathrm{Fe}\end{array}$ & $3\}$ & $\cdots$ & 7 & $\cdots$ & $\odot^{\prime \prime}$ & $\ldots$ & 10 & ...... & $5424 \cdot 284$ \\
\hline & & & & & & & & & \\
\hline
\end{tabular}

* The distance apart of the components of this 1474 line measured accurately is 0.146 by Crew, and 0.141 by Rowland. The coinoidences with Fe and $C_{0}$ are very doubtful. The Co line comes more nearly between the two rather than coineiding with either. (R.)

$A$ trial of substances in the are gave the following results :-iron, manganese, chromium, titanium, and two different specimens of meteoric stones showed two faint lines baving the same relative intensities with respect to each other as the components of 1474 in the solar spectrum, and either coincided with the components of 1474 or nearly so. When cobalt and nickel were tried, the lines were the same distance apart but the relative intensities were reversed, the red component being much the stronger.

With the best definition 1474 is a triple, or rather a double the red nomponent of which has a weak side line to violet. The componente as measured on a photographic plate are respectively 0.120 and 0.050 apart. The main components as determined by a series of measurements on photographic plates are $0 \cdot 170$ apart.

Probably the violet component of the double is iron and the weak side line of the red component is cobalt, but the red component is unknown. (J.)

+ With but little material in the arc this is a diffeult triplet. The violet component is very strong, the red component about half as strong, and between them, but nearer the red component, is a very narrow line much weaker than either of the others. (J.)

$\ddagger$ There are some fine lines near to red side.

$\$$ Components about $0 \cdot 110$ apart on photographic plates. (J.)

II Red component itself is an exceedingly diffeult double. (J.) 


\begin{tabular}{|c|c|c|c|c|c|c|c|c|c|}
\hline \multirow{2}{*}{ lements. } & \multicolumn{2}{|c|}{ IN ARC. } & \multicolumn{2}{|c|}{ IN Sun. } & \multirow{2}{*}{$\begin{array}{l}\text { Kind } \\
\text { of } \\
\text { Stand- } \\
\text { ard. }\end{array}$} & \multicolumn{2}{|c|}{ Wright. } & \multirow{2}{*}{$\begin{array}{l}\text { Ware- } \\
\text { length } \\
\text { in Arc. }\end{array}$} & \multirow{2}{*}{$\begin{array}{l}\text { Wave- } \\
\text { length } \\
\text { in Sun. }\end{array}$} \\
\hline & $\begin{array}{c}\text { Inten- } \\
\text { sity. }\end{array}$ & $\begin{array}{c}\text { Appear- } \\
\text { ance. }\end{array}$ & $\begin{array}{c}\text { Inten- } \\
\text { sity. }\end{array}$ & $\begin{array}{l}\text { Appear- } \\
\text { ance. }\end{array}$ & & $\underset{\text { Arc. }}{\operatorname{In}}$ & In & & \\
\hline $\mathrm{Fe}$ & 5 & $\cdots$ & 6 & $\cdots$ & $\odot^{\prime \prime}$ & 1 & 9 & $5434 \cdot 725$ & 5434 \\
\hline$\ldots$ & 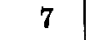 & $\ldots$ & 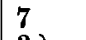 & $\cdots$ & $\bigodot^{\prime \prime}$ & 1 & 9 & 5447 & \\
\hline Fe? ] ... & $\cdots$ & $\ldots$ & 3 & $\dddot{\alpha}$ & $\bigodot_{1}$ & $\ldots$ & 1 & ...... & \\
\hline $\mathrm{Fe}$ & 6 & $\ldots$ & 6 & $\begin{array}{l}a \\
\ldots\end{array}$ & $\stackrel{\odot}{\odot}^{\prime}$ & $\cdots$ & 1 & …... & \\
\hline $\mathrm{Ni}$ & 3 & $\ldots$ & 1 & 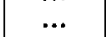 & $\bigodot^{\prime}$ & $\ldots$ & 7 & …... & 546 \\
\hline Fe $\ldots . .$. & 3 & $\ldots$ & 4 & & $\bigodot^{\prime \prime}$ & $\ldots$ & 9 & ....... & 546 \\
\hline $\mathrm{Fe} \quad \ldots . .$. & 3 & $\ldots$ & 4 & & $\odot^{\prime \prime}$ & $\ldots$ & 10 & ........ & 54 \\
\hline $\mathbf{F}$ & 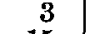 & & 0 & $\cdots$ & $\bigodot^{\prime \prime}$ & $\ldots$ & 10 & ....... & \\
\hline $\mathrm{Ni} \quad \ldots . .$. & 15 & $\mathbf{R}$ & 4 & 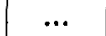 & $\odot^{\prime \prime}$ & $\ldots$ & 10 & ....... & 547 \\
\hline Fe $\ldots . .$. & 8 & $\ldots$ & 3 & $\cdots$ & $\odot^{\prime}$ & $\ldots$ & 5 & ...... & \\
\hline .. & 3 & $\ldots$ & 4 & $\ldots$ & $\odot^{\prime \prime}$ & $\ldots$ & 8 & ....... & 54 \\
\hline ....... & 5 & $\ldots$ & 4 & $\ldots$ & $\odot^{\prime \prime}$ & $\ldots$ & 8 & ...... & \\
\hline $\mathrm{Fe} .$. & 5 & & 4 & $\ldots$ & $\bigodot^{\prime \prime}$ & & 8 & & 550 \\
\hline & 5 & $\mathbf{N}$ & 3 & $\ldots$ & $\odot$ & 1 & 8 & $\mid 5513 \cdot 127$ & 551 \\
\hline Mg†...... & 10 & .. & 7 & $\cdots$ & $\bigodot^{\prime \prime}$ & 4 & 8 & $5528 \cdot 672$ & 55 \\
\hline ....... & 8 & & $?$ & . & $\bigodot^{\prime \prime}$ & $\cdots$ & 8 & ....... & 55 \\
\hline F & 3 & & 2 & & $\bigodot^{\prime \prime}$ & $\ldots$ & 8 & & \\
\hline $\mathbf{F}$ & 3 & & 2 & $\ldots$ & $\bigodot^{\prime \prime}$ & $\ldots$ & 9 & ........ & \\
\hline$\ldots$ & 4 & & 3 & 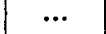 & $\odot^{\prime}$ & $\ldots$ & 8 & ....... & \\
\hline ....... & 6 & 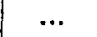 & 5 & $\ldots$ & $\odot^{\prime \prime}$ & $\ldots$ & 8 & ....... & \\
\hline $\mathbf{F}$ & 5 & & 4 & $\ldots$ & $\bigodot^{\prime \prime}$ & $\ldots$ & 7 & & \\
\hline C & 6 & & 4 & $\ldots$ & $\mathbf{M} \odot^{\prime \prime}$ & 2 & 9 & 5582:204 & \\
\hline $\mathrm{Ca}$ & 10 & $\mathbf{R}$ & 6 & $\ldots$ & $\mathbf{M} \odot^{\prime \prime}$ & 2 & 9 & 5588 & \\
\hline $\mathrm{Oa}$. & 5 & & 4 & ... & $\mathbf{M} \odot^{\prime \prime}$ & 2 & 5 & 559 & \\
\hline ....... & 7 & $\mathbf{R}$ & 5 & $\ldots$ & M॰ & 2 & 5 & 559 & \\
\hline ....... & 3 & ... & 2 & $d$ & $\odot^{\prime}$ & 1 & 2 & 559 & \\
\hline C & 7 & & 4 & & $\odot^{\prime}$ & 2 & 4 & 559 & \\
\hline O & 5 & . & 4 & $\cdots$ & $\overline{\mathbf{M}} \odot{ }^{\prime \prime}$ & 2 & 4 & $|5601 \cdot 502|$ & $5601 \cdot 50$ \\
\hline & 2 & & 2 & & & & & & \\
\hline$\$ \cdots$ & 6 & $\cdots$ & & $\mathrm{t}$ & $\odot^{\prime}$ & $\cdots$ & 10 & ...... & $5603 \cdot 09^{r}$ \\
\hline $\mathrm{Fe} \quad . . . .$. & $\begin{array}{l}5 \\
2\end{array}$ & - & $\begin{array}{l}b \\
2\end{array}$ & 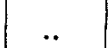 & $\odot^{\prime}$ & & 10 & & \\
\hline & 2 & . & 6 & ... & $\odot^{\prime}$ & .. & 10 & ....... & \\
\hline & 2 & & 2 & $\cdots$ & $\odot^{\prime \prime}$ & $\because$ & 12 & ....... & \\
\hline $\mathrm{Fe}-\mathrm{Va} \|$ & $\begin{array}{ll}5 & 2\end{array}$ & .. & 4 & $\ldots$ & $\odot^{\prime \prime}$ & .. & 14 & ....... & \\
\hline ...... & 2 & & 3 & 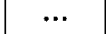 & $\odot^{\prime \prime}$ & & 5 & ....... & 563 \\
\hline ...... & 2 & & 3 & $\ldots$ & $\bigodot^{\prime \prime}$ & 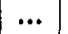 & 10 & $\ldots \ldots$ & \\
\hline 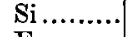 & $\ddot{g}$ & & 2 & .. & 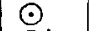 & & 9 & $\ldots$. & \\
\hline & 2 & & 4 & .. & $\bigodot^{\prime}$ & .. & 9 & ....... & \\
\hline $\mathbf{Y}$ & 1 & & 4 & & $\bigodot^{\prime \prime}$ & & $y$ & ....... & \\
\hline $\mathrm{F}$ & $\mathbf{3}$ & & 5 & ... & $\odot^{\prime \prime}$ & $\ldots$ & 9 & ....... & 566 \\
\hline Ti......... & 3 & 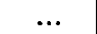 & 2 & ... & $\odot$ & 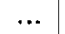 & 8 & ....... & \\
\hline $\mathrm{Fe}$ & 2 & ... & 3 & $\ldots$ & $\bigodot^{\prime \prime}$ & $\ldots$ & 8 & ....... & \\
\hline $\mathrm{Na}$ & 3 & $\cdots$ & 4 & $\cdots$ & $\odot^{\prime \prime}$ & $\cdots$ & 9 & $\ldots \ldots$. & $5682 \cdot 861$ \\
\hline
\end{tabular}

* A difficult double. (J.)

+ This $\mathrm{Mg}$ line is shaded to one side when there is much $\mathrm{Mg}$ in the arc and is therefore a poor metallic standard. The solar line corresponds to the extreme edge of this band-like line. (R.)

$\ddagger$ There is a side line towards the violet.

8 This triplet is made up of close double and a line close to red stronger than either component of double ; wave-length of components about 5602.995; 5603.080 and 5603.180 as measured on a photographic plate. (J.)

If Lines used by Perreo in his determinations of absolute wave-lengths. 


\begin{tabular}{|c|c|c|c|c|c|c|c|c|c|}
\hline \multirow{2}{*}{ Elements. } & \multicolumn{2}{|c|}{ In ARo. } & \multicolumn{2}{|c|}{ In Sun. } & \multirow{2}{*}{$\begin{array}{l}\text { Kind } \\
\text { of } \\
\text { Stand- } \\
\text { ard. }\end{array}$} & \multicolumn{2}{|c|}{ W EIGHT. } & \multirow{2}{*}{$\begin{array}{l}\text { Wave- } \\
\text { length } \\
\text { in Arc. }\end{array}$} & \multirow{2}{*}{$\begin{array}{l}\text { Wave- } \\
\text { length } \\
\text { in Sun. }\end{array}$} \\
\hline & $\begin{array}{l}\text { Inten- } \\
\text { sity. }\end{array}$ & $\begin{array}{c}\text { Appear- } \\
\text { ance. }\end{array}$ & $\begin{array}{l}\text { Inten } \\
\text { sity. }\end{array}$ & $\begin{array}{c}\text { Appear- } \\
\text { ance. }\end{array}$ & & $\begin{array}{c}\text { In } \\
\text { Arc. }\end{array}$ & $\mid \begin{array}{c}\text { In } \\
\text { Sun. }\end{array}$ & & \\
\hline $\mathrm{Na}$ & & & 6 & ... & $\odot^{\prime \prime}$ & & 7 & & $688 \cdot 434$ \\
\hline $\mathrm{Fe} \quad \ldots . .$. & $\ddot{4}$ & ... & 0 & $\cdots$ & $\bigodot^{\prime \prime}$ & $\cdots$ & 8 & ….... & \\
\hline $\mathrm{Si} \ldots \ldots \ldots$ & $\cdots$ & ... & 5 & ... & $\bigodot^{\prime \prime}$ & $\ldots$ & 4 & ....... & $5708 \cdot 620$ \\
\hline $\begin{array}{ll}\text { Fe } & \ldots . . . \\
\mathrm{Ni} & \ldots . . .\end{array}$ & $\ddot{r}$ & $\cdots$ & $6\}$ & d & $\odot$ & $\cdots$ & 6 & ....... & 570 \\
\hline $\mathrm{Mg} * \ldots$ & $\boldsymbol{b}$ & $\ldots$ & $\begin{array}{l}5] \\
6\end{array}$ & $\ldots$ & $\stackrel{\ominus}{\mathbf{M} \odot}$ & $\dddot{4}$ & 8 & 5711.374 & $\begin{array}{l}9.76 \\
1.318\end{array}$ \\
\hline $\mathrm{Ni}$ & $\ddot{5}$ & & & $\cdots$ & & & $\cdots$ & & $711 \cdot 318$ \\
\hline $\mathrm{Fe}-\mathrm{Ti}\}$ & $3\}$ & $\cdots$ & 5 & $\cdots$ & $\bigodot^{\prime \prime}$ & $\cdots$ & 10 & ....... & $5715 \cdot 309$ \\
\hline $\begin{array}{ll}\mathrm{Fe} & \ldots . . . \\
\mathrm{Fe} & \ldots . . .\end{array}$ & $\ldots$ & ... & 5 & ... & $\bigodot^{\prime \prime}$ & $\ldots$ & 10 & $\ldots \ldots$ & 5731.97 \\
\hline $\begin{array}{ll}\mathrm{Fe} & \ldots . . \\
\mathrm{Fe} & \ldots . . .\end{array}$ & $\cdots$ & $\cdots$ & 3 & $\cdots$ & ఠ & $\ldots$ & 10 & ....... & 574 \\
\hline $\begin{array}{l}\text { Fe } \\
\text { Fe }\end{array}$ & $\cdots$ & $\cdots$ & 4 & $\cdots$ & $\bigodot^{\prime \prime}$ & $\cdots$ & 10 & ....... & 257 \\
\hline & '. & $\cdots$ & 5 & $\cdots$ & $\odot^{\prime \prime}$ & $\cdots$ & 10 & ...... & $5753 \cdot 342$ \\
\hline & $\cdots$ & $\cdots$ & 5 & $\cdots$ & $\odot^{\prime \prime}$ & $\cdots$ & 9 & ...... & $5754 \cdot 884$ \\
\hline $\begin{array}{l}\text { Fet ....... } \\
\text { Si .......... }\end{array}$ & $\cdots$ & $\cdots$ & 7 & $\cdots$ & $\bigodot_{1}$ & $\cdots$ & 8 & ...... & $5763 \cdot 215$ \\
\hline $\mathrm{Fe} \quad \ldots . . .$. & $\ldots$ & $\cdots$ & 5 & ... & $\bigodot^{\prime \prime}$ & $\ldots$ & 6 & ....... & $5772 \cdot 360$ \\
\hline Cu?Co? & ... & $\cdots$ & $\begin{array}{l}5 \\
7\end{array}$ & d? & $\bigodot_{\odot}^{\prime \prime}$ & $\cdots$ & 9 & ...... & 304 \\
\hline Cr $\quad \ldots$ & 6 & $\cdots$ & 4 & do & $\stackrel{\odot}{\odot}^{\prime \prime}$ & $\cdots$ & $\begin{array}{l}9 \\
9\end{array}$ & $\cdots, \cdot \cdot$ & $\begin{array}{l}5782 \cdot 346 \\
5784 \cdot 081\end{array}$ \\
\hline $\mathrm{Cr}$ & 7 & ... & 5 & $\cdots$ & $\bigodot^{\prime \prime}$ & $\ldots$ & 13 & …..... & $5788 \cdot 136$ \\
\hline $\left.\begin{array}{l}\mathrm{Cr} \\
\mathrm{Fe}\end{array}\right\}$ & 10 & $\cdots$ & 7 & $d ?$ & $\odot^{\prime \prime}$ & $\ldots$ & 16 & ...... & $5791 \cdot 207$ \\
\hline ? & $\ldots$ & $\ldots$ & 4 & $\ldots$ & $\odot^{\prime \prime}$ & $\ldots$ & 10 & 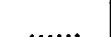 & 5798.087 \\
\hline $\mathrm{Fe}$. & $\ldots$ & $\cdots$ & 5 & & $\bigodot^{\prime \prime}$ & $\cdots$ & 9 & ........ & \\
\hline $\mathrm{Ni} \quad \ldots . .$. & 7 & $\ldots$ & 5 & $\ldots$ & $\bigodot^{\prime \prime}$ & $\ldots$ & 8 & ........ & 580 \\
\hline Fe $\quad \ldots . .$. & $\ldots$ & $\ldots$ & 5 & $\ldots$ & $\bigodot^{\prime \prime}$ & $\ldots$ & .7 & ........ & $5806 \cdot 95$ \\
\hline Fe ... & ... & $\ldots$ & 5 & $\ldots$ & $\bigodot^{\prime \prime}$ & $\ldots$ & 14 & ....... & $5809 \cdot 43$ \\
\hline Fe $\ddagger$. & $\ddot{g}$ & $\cdots$ & 6 & ". & $\bigodot_{1}$ & $\ldots$ & .14 & ........ & 58 \\
\hline $\mathbf{N}$ & 3 & .. & 3 & $\cdots$ & $\odot^{\prime}$ & $\ldots$ & 6 & ....... & 58 \\
\hline $\mathrm{Ba}$ & 10 & $\cdots$ & 5 & & $\odot^{\prime \prime}$ & $\cdots$ & 14 & ....... & \\
\hline $\mathrm{Ca} \S \ldots . . .$. & 10 & $\ldots$ & 7 & ... & $\odot^{\prime}$ & $\ldots$ & 14 & ....... & 58 \\
\hline $\mathrm{Fe} \ldots . .$. & $\cdots$ & $\ldots$ & 6 & .. & $\bigodot^{\prime \prime}$ & $\ldots$ & 15 & ...... & 58 \\
\hline$F_{\theta}$ & ... & $\ldots$ & 6 & $\ldots$ & $\odot^{\prime \prime}$ & $\ldots$ & 16 & ....... & $5862 \cdot 5$ \\
\hline $\mathbf{D}_{3} \mathrm{He} \| \ldots$ & ... & $\cdots$ & & $\cdots$ & $\cdots$ & $\cdots$ & ... & ....... & $5875 \cdot 982$ \\
\hline $\left.\begin{array}{l}\mathrm{Fe} \\
\mathrm{A}(\mathrm{wr})\end{array}\right\}$ & $\cdots$ & $\cdots$ & $\left.\begin{array}{l}6 \\
4\end{array}\right\}$ & $d$ & $\bigodot_{1}$ & $\ldots$ & 11 & ....... & 5884.04 \\
\hline$A(w$ & $\cdots$ & $\cdots$ & 3 & $\cdots$ & $\odot_{1}$ & ... & 8 & & \\
\hline $\mathrm{D}_{2} \mathrm{Na}^{\prime} \ldots \ldots$ & $\ldots$ & $\ldots$ & 15 & $\ldots$ & $\odot^{\prime}$ & $\cdots$ & 20 & ......... & $\mathbf{5 8 9 0} \cdot 18$ \\
\hline $\mathrm{Ni}^{* *} \ldots \ldots$ & 3 & $d ?$ & 4 & $\cdots$ & $\odot$ & $\cdots$ & 14 & ........ & 5893.09 \\
\hline
\end{tabular}

* This $\mathrm{Mg}$ line is shaded on one side, especially when there is much $\mathrm{Mg}$ in the arc and therefore should not be used for a metallie standard. (R.)

$\dagger$ There is a fine line near to violet side.

$\$$ There is a line close to violet side.

$\S$ There is a Ni line near to red.

II This value of the wave-length of $D_{3}$ is the result of three serjes of measurements made with a grating having 20,000 , lines to the inch, and is accurate to perhaps 0.02 .

Observations were made in the first spectrum on botb sides of the Sun. The line does not occur as a dark line in the solar spectrum; but is sometimes, if not always, present as a rery weak bright line. This is shown by a study of the best photographs of this region of the solar spectrum. (J.)

I A water-vapour line is toward the red about 0.080 from the $\mathrm{Fe}$ line and ordinarily forms a double with it.

** An exceedingly close equal double when there is rery good definition. There is also a solar line near to the violet side, and a water-rapour line near to the red. (J.) 
Prof. H. A, Rowland on a

\begin{tabular}{|c|c|c|c|c|c|c|c|c|c|}
\hline \multirow{2}{*}{ Flementa. } & \multicolumn{2}{|c|}{ IN ARC. } & \multicolumn{2}{|c|}{ In Sun. } & \multirow{2}{*}{$\begin{array}{c}\text { Kind } \\
\text { of } \\
\text { Stand- } \\
\text { ard. }\end{array}$} & \multicolumn{2}{|c|}{ Whight. } & \multirow{2}{*}{$\begin{array}{l}\text { Wave- } \\
\text { length } \\
\text { in Arc. }\end{array}$} & \multirow{2}{*}{$\begin{array}{l}\text { Wave- } \\
\text { length } \\
\text { in Sur. }\end{array}$} \\
\hline & $\begin{array}{l}\text { Inten- } \\
\text { sity. }\end{array}$ & Appear- & $\begin{array}{l}\text { Inten- } \\
\text { sity. }\end{array}$ & $\begin{array}{c}\text { Appear } \\
\text { ance. }\end{array}$ & & $\begin{array}{l}\text { In } \\
\text { Are. }\end{array}$ & Sun. & & \\
\hline $\mathrm{D}_{1} \mathbf{N a}$ & $\cdots$ & $\cdots$ & 10 & $\cdots$ & $\odot^{\prime \prime}$ & $\ldots$ & 20 & ....... & $5896 \cdot 154$ \\
\hline$A(w v)$ & ... & $\ldots$ & $3\}$ & $\mathrm{d} ?$ & $\odot_{1}$ & $\ldots$ & 10 & $\ldots .$. & $5898 \cdot 39$ \\
\hline & $\cdots$ & $\cdots$ & $\frac{1}{5}$ & & & & & & \\
\hline $\overrightarrow{\mathbf{A}}$ & $\begin{array}{l}\cdots \\
\ldots\end{array}$ & $\cdots$ & $\left.\begin{array}{l}0 \\
1\end{array}\right\}$ & d & $\odot_{1}$ & $\cdots$ & 13 & ....... & $5901 \cdot 681$ \\
\hline & $\ldots$ & ... & 5 & $\cdots$ & $\Theta^{\prime \prime}$ & $\ldots$ & 15 & ....... & 590589 \\
\hline Fo $\ldots\}^{*}$ & $\cdots$ & $\cdots$ & 4 & $d$ & $\odot^{\prime \prime}$ & $\ldots$ & 17 & ....... & 5914.38 \\
\hline & ... & $\cdots$ & 5 & $\cdots$ & $\odot^{\prime \prime}$ & .. & 16 & ....... & $5916 \cdot 47$ \\
\hline$A(w)$ & ... & ... & 6 & ... & $\bigodot^{\prime \prime}$ & ... & 12 & ....... & \\
\hline $\mathrm{Fe}$ & $\ldots$ & $\ldots$ & 6 & $\ldots$ & $\bigodot^{\prime \prime}$ & ... & 14 & ....... & 593 \\
\hline Fe & $\ldots$ & $\ldots$ & 6 & ... & $\bigodot^{\prime \prime}$ & $\ldots$ & 13 & ....... & \\
\hline $\mathbf{S i}, \ldots$ & $\ldots$ & ... & 6 & ... & $\Theta^{\prime \prime}$ & ... & 14 & ....... & \\
\hline $\mathrm{Fe}$ & $\ldots$ & $\ldots$ & 5 & ... & $\bigodot^{\prime \prime}$ & $\ldots$ & 12 & ....... & \\
\hline $\mathrm{F}_{0}$. & $\cdots$ & $\ldots$ & 4 & $\ldots$ & $\bigodot^{\prime \prime}$ & .. & 12 & ....... & \\
\hline & $\ldots$ & .. & 5 & $\ldots$ & $\odot$ & $\ldots$ & 13 & ....... & 597 \\
\hline $\mathbf{A}(\mathbf{w}) \ldots$ & $\ldots$ & $\ldots$ & 2 & $\ldots$ & $\bigodot_{2}$ & $\ldots$ & 1 & ....... & \\
\hline $\mathrm{Fe}$ & $\ldots$ & $\ldots$ & 6 & $\ldots$ & $\bigodot^{\prime \prime}$ & ... & 6 & ....... & 598 \\
\hline$F_{\theta}$ & $\ldots$ & $\ldots$ & 6 & $\ldots$ & $\bigodot^{\prime \prime}$ & $\cdots$ & 7 & ....... & \\
\hline $\mathrm{Fe}$ & $\cdots$ & $\ldots$ & 6 & $\ldots$ & $\bigodot^{\prime \prime}$ & $\ldots$ & 7 & ....... & 60 \\
\hline Fe $\ldots . .$. & $\ldots$ & $\cdots$ & 4 & $\ldots$ & $\bigodot^{\prime \prime}$ & $\ldots$ & 3 & ....... & \\
\hline$F_{\theta}$ & & $\ldots$ & 6 & $\ldots$ & $\bigodot^{\prime \prime}$ & .. & 6 & $\ldots .$. & 60 \\
\hline $\mathbf{M n}$ & 10 & $\cdots$ & 6 & $\ldots$ & $\bigodot^{\prime \prime}$ & ... & 5 & ....... & \\
\hline $\mathbf{M n}$ & 10 & ... & 6 & $\ldots$ & $\odot "$ & $\cdots$ & 8 & $\ldots \ldots$ & \\
\hline$?\}^{\dagger} .$. & $\cdots$ & 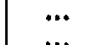 & $\left.\begin{array}{l}3 \\
5\end{array}\right\}$ & $d$ & $\odot_{1}$ & ... & 6 & ...... & $6020 \cdot 347$ \\
\hline $\mathrm{MU}_{n}$ & 10 & ... & 5 & .. & $\odot^{\prime \prime}$ & $\cdots$ & 6 & ......, & 602 \\
\hline$F_{\theta} \ldots . .$. & $\ldots$ & $\ldots$ & 6 & $\ldots$ & $\bigodot^{\prime \prime}$ & $\ldots$ & 8 & ....... & \\
\hline Fe ....... & $\cdots$ & . & 4 & ... & $\odot^{\prime \prime}$ & $\ldots$ & 7 & ....... & 60 \\
\hline $\mathrm{Fe} \quad \ldots \ldots$ & $\cdots$ & .. & $\underline{4}$ & 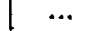 & $\bigodot^{\prime \prime}$ & $\cdots$ & 8 & ....... & \\
\hline$F \theta$ & $\cdots$ & .. & 5 & $\cdots$ & ๑" & $\ldots$ & 9 & ........ & \\
\hline Fe $\ldots . .$. & $\ldots$ & 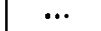 & 7 & $\ldots$ & $\bigodot^{\prime \prime}$ & $\ldots$ & 13 & ....... & \\
\hline Fe $\quad . . . .$. & 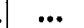 & ... & 5 & $\ldots$ & $\odot^{\prime \prime}$ & ... & 13 & ....... & 60 \\
\hline $\mathrm{Fe} \quad \ldots . .$. & $\ldots$ & $\ldots$ & 3 & $\ldots$ & $\bigodot^{\prime \prime}$ & $\ldots$ & 12 & ........ & \\
\hline & 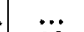 & . & 4 & $\ldots$ & $\odot^{\prime}$ & $\ldots$ & 4 & ....... & 610 \\
\hline $\mathrm{Ca}$ & 10 & $\mathbf{R}$ & 6 & $\ldots$ & 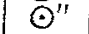 & $\ldots$ & 9 & ....... & 6102941 \\
\hline Fe & $\cdots$ & $\ldots$ & 4 & d & $\bigodot_{1}$ & $\ldots$ & 8 & ........ & 6103.449 \\
\hline$\stackrel{?}{\mathrm{ti}}$ & 20 & $\cdots$ & 1) & $\ldots$ & $\mathrm{M}$ & 4 & & $6103 \cdot 812$ & \\
\hline $\mathrm{Ni} \ldots . .$. & 5 & $\cdots$ & $\dddot{6}$ & $\cdots$ & $Q^{\prime \prime}$ & $\ldots$ & $\ddot{8}$ & ....... & 610 \\
\hline ......... & 4 & .. & 3 & $\ldots$ & $\odot^{\prime}$ & $\ldots$ & 8 & ........ & \\
\hline & 5 & $\cdots$ & 6 & $\cdots$ & $\bigodot^{\prime \prime}$ & ... & 8 & ....... & \\
\hline $\mathrm{Ca}$ & 15 & $\mathbf{R}$ & 9 & $\ldots$ & $\odot^{\prime \prime}$ & $\ldots$ & 11 & ....... & \\
\hline $\mathrm{Fe}$ & & & 8 & $\cdots$ & $\widehat{\odot}^{\prime}$ & $\ldots$ & 9 & ....... & 613 \\
\hline$F_{\theta}-B_{a} \ldots$ & $?-15$ & $\ldots$ & 7 & $\ldots$ & $\bigodot^{\prime \prime}$ & $\ldots$ & 9 & ........ & 614 \\
\hline $\mathbf{N}$ & 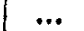 & $\ldots$ & 3 & $\cdots$ & $\bigodot^{\prime \prime}$ & $\cdots$ & 5 & ....... & 615 \\
\hline$\hat{N}$ & 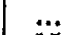 & 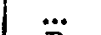 & 5 & $\ldots$ & $\bigodot^{\prime \prime}$ & $\ldots$ & 4 & ....... & 6160 \\
\hline $\mathrm{C}$ & 15 & $\mathbf{R}$ & 10 & ... & $\odot^{\prime \prime}$ & ... & 9 & $\ldots \ldots$ & 6162 \\
\hline $\mathrm{Ca}$ & 6 & $\ldots$ & 6 & $\ldots$ & $\bigodot^{\prime \prime}$ & $\ldots$ & 4 & ....... & 616 \\
\hline $\mathrm{Ca}$ & 7 & & 7 & $\cdots$ & $\odot^{\prime \prime}$ & .. & 8 & ....... & $6169 \cdot 7$ \\
\hline
\end{tabular}

* Componente about 0-100 apart. Red component is partly solar and partly water-vapour. (J.)

+ Oomponents about 0.200 apart.

$\ddagger$ Components 0*100 apart. 


\begin{tabular}{|c|c|c|c|c|c|c|c|c|c|}
\hline \multirow{2}{*}{ Elements. } & \multicolumn{2}{|c|}{$\mathrm{I}_{\mathrm{N}}$ ARC. } & \multicolumn{2}{|c|}{ In Sun. } & \multirow{2}{*}{$\begin{array}{c}\text { Kind } \\
\text { of } \\
\text { Stand- } \\
\text { ard. }\end{array}$} & \multicolumn{2}{|c|}{ Wеiबнт. } & \multirow{2}{*}{$\begin{array}{l}\text { Wave- } \\
\text { length. } \\
\text { in Arc. }\end{array}$} & \multirow{2}{*}{$\begin{array}{l}\text { Wave- } \\
\text { length } \\
\text { in Sun. }\end{array}$} \\
\hline & $\begin{array}{c}\text { Inten- } \\
\text { sity. }\end{array}$ & $\begin{array}{c}\text { Appear- } \\
\text { ance. }\end{array}$ & $\begin{array}{l}\text { Inten- } \\
\text { sity. }\end{array}$ & $\begin{array}{c}\text { Appear- } \\
\text { ance. }\end{array}$ & & $\begin{array}{l}\text { In } \\
\text { Arc. }\end{array}$ & $\begin{array}{c}\text { In } \\
\text { Snn. }\end{array}$ & & \\
\hline $\mathrm{Fe}$ & ... & $\cdots$ & 6 & $\ldots$ & $\odot^{\prime \prime}$ & $\ldots$ & 8 & ....... & $6173: 5$ \\
\hline $\mathrm{Ni}$ & 5 & $\ldots$ & 6 & & $\bigodot^{\prime \prime}$ & $\ldots$ & 8 & ....... & \\
\hline$F_{\theta} \quad \ldots \ldots$ & H. & $\ldots$ & 6 & $\ldots$ & $\bigodot^{\prime \prime}$ & $\ldots$ & 8 & ....... & \\
\hline $\mathrm{Ni} \quad \ldots .$. & 4 & $\ldots$ & 6 & $\ldots$ & $\odot^{\prime \prime}$ & $\ldots$ & 9 & ........ & 619 \\
\hline$F_{\theta} \quad \ldots . .$. & $\cdots$ & $\ldots$ & 8 & $\ldots$ & $\odot^{\prime \prime}$ & $\ldots$ & 10 & $\ldots \ldots$ & \\
\hline$F_{\theta} \quad \ldots \ldots$ & .. & $\ldots$ & 6 & $\ldots$ & $\odot^{\prime \prime}$ & $\ldots$ & 10 & ....... & \\
\hline$\ldots \ldots$ & $\ldots$ & $\ldots$ & 6 & $\ldots$ & $\bigodot^{\prime \prime}$ & $\ldots$ & 9 & ....... & 21 \\
\hline $\mathrm{Fe}$ & & .. & 6 & & $\bigodot^{\prime \prime}$ & $\ldots$ & 10 & ....... & 621 \\
\hline$F_{\theta}-\nabla_{a} \ldots$ & $?-6$ & $\ldots$ & 7 & $\cdots$ & $\bigodot^{\prime \prime}$ & $\ldots$ & 12 & ....... & 623 \\
\hline$? \quad \ldots \ldots$ & $\ldots$ & $\ldots$ & 4 & $\ldots$ & $\bigodot^{\prime \prime}$ & $\ldots$ & 8 & ....... & 6237 \\
\hline Fe $\ldots . .$. & $\ldots$ & $\ldots$ & 7 & $\ldots$ & $\bigodot^{\prime \prime}$ & $\ldots$ & 9 & ....... & 6246 \\
\hline & $\ldots$ & $\ldots$ & 7 & $\ldots$ & $\bigodot^{\prime \prime}$ & $\ldots$ & 9 & ....... & 625 \\
\hline$F_{\theta^{*}}$ & & 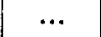 & 7 & ... & $\bigodot^{\prime \prime}$ & $\ldots$ & 9 & ...... & 625 \\
\hline $\mathrm{Ni}\}$ & $7\}$ & ... & 6 & $\ldots$ & $\odot^{\prime \prime}$ & $\ldots$ & 8 & ....... & $6256: 57$ \\
\hline $\begin{array}{l}\mathrm{Fe} J \\
\mathrm{Ti}\end{array}$ & $\frac{?}{5}$ & ... & 2 & $\ldots$ & $\bigodot^{\prime \prime}$ & $\ldots$ & 9 & ....... & $6261 \cdot 31$ \\
\hline $\mathrm{Fe}$. & $\ldots$ & $\cdots$ & 5 & ... & $\odot^{\prime \prime}$ & $\ldots$ & 11 & ........ & 626 \\
\hline & $\ldots$ & $\ldots$ & 3 & & $\bigodot^{\prime \prime}$ & $\ldots$ & 10 & ........ & 6270 \\
\hline$\alpha A(O) \dagger \ldots$ & 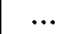 & .. & 4 & d & $\odot^{\prime \prime}$ & .. & 9 & ....... & 6278 \\
\hline $\mathrm{A}(\mathrm{O}) \quad \ldots$ & $\ldots$ & $\ldots$ & 2 & $\cdots$ & $\bigodot^{\prime}$ & $\cdots$ & 7 & ....... & 628 \\
\hline$\cdots$ & $\ldots$ & $\ldots$ & 2 & $\ldots$ & $\bigodot^{\prime}$ & $\ldots$ & 5 & ....... & 628 \\
\hline $\mathbf{A}(0) \S \ldots$ & $\ldots$ & .. & 3 & $\ldots$ & $\bigodot_{\mu}$ & $\ldots$ & 6 & ...... & 629 \\
\hline )) $\ldots$ & $\ldots$ & $\cdots$ & 3 & $\ldots$ & $\odot^{\prime \prime}$ & ... & 7 & ....... & 629 \\
\hline & $\cdots$ & $\ldots$ & 7 & $\ldots$ & $\odot^{\prime \prime}$ & $\ldots$ & 7 & ....... & \\
\hline & 6 & $\cdots$ & 4 & $\ldots$ & $\bigodot^{\prime \prime}$ & $\ldots$ & 7 & ....... & 6 \\
\hline & $\cdots$ & $\ldots$ & 3 & $\cdots$ & $\bigodot_{1}$ & $\ldots$ & 5 & ....... & 63 \\
\hline (Ca). & $\ldots$ & $\cdots$ & 6 & $\ldots$ & $\bigodot^{\prime \prime}$ & $\ldots$ & 14 & ....... & \\
\hline & $\ldots$ & $\cdots$ & 5 & $\ldots$ & $\bigodot^{\prime \prime}$ & $\ldots$ & $\cdot 13$ & ....... & 6 \\
\hline F & . & $\ldots$ & 6 & $\ldots$ & $\odot^{\prime \prime}$ & $\ldots$ & $\cdot 12$ & ....... & \\
\hline & .. & $\ldots$ & 6 & $\ldots$ & $\bigodot^{\prime \prime}$ & $\cdots$ & 12 & $\ldots . .$. & \\
\hline & 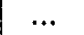 & $\ldots$ & 5 & $\ldots$ & $\bigodot^{\prime \prime}$ & $\ldots$ & 6 & ........ & \\
\hline $\mathrm{Fe} q_{1} \ldots . .$. & .. & $\ldots$ & 5 & $\ldots$ & ஓ & $\ldots$ & 8 & ....... & \\
\hline$\ldots \ldots$ & & $\ldots$ & 6 & $\cdots$ & $\bigodot^{\prime \prime}$ & $\ldots$ & 8 & 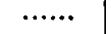 & \\
\hline $\mathrm{Ni}$ & 5 & $\cdots$ & 2 & $\cdots$ & $\bigodot_{1}$ & $\ldots$ & 2 & ...... & \\
\hline $\mathbf{F}$ & ... & $\cdots$ & 4 & $\cdots$ & $\bigodot^{\prime \prime}$ & $\ldots$ & 6 & ...... & \\
\hline $\mathbf{F}$ & $\ldots$ & $\cdots$ & 7 & $\cdots$ & $\bigodot^{\prime \prime}$ & & 9 & ...... & \\
\hline$\ldots \ldots$ & .. & $\ldots$ & 8 & $\ldots$ & $\bigodot_{1}$ & . & 5 & ....... & \\
\hline$F$ & $\because$ & ... & 3 & $\ldots$ & $\bigodot_{1}$ & 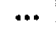 & 6 & ...... & \\
\hline ...... & $\cdots$ & $\ldots$ & 6 & $\cdots$ & $\bigodot^{\prime \prime}$ & $\ldots$ & 8 & ....... & \\
\hline $\mathbf{F}$ & .. & $\cdots$ & 7 & ... & $\odot^{\prime \prime}$ & $\cdots$ & 10 & ....... & \\
\hline & 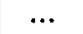 & ... & 5 & $\cdots$ & $\bigodot^{\prime \prime}$ & o. & 8 & ....... & \\
\hline $\mathrm{Fe}^{* *} \ldots$ & $\ldots$ & ... & 6 & ... & ○. & $\ldots$ & 10 & ....... & 6421 \\
\hline$F_{E}$ & $\cdots$ & $\ldots$ & 6 & ... & $\odot^{\prime \prime}$ & $\because$ & 10 & & 6431.0 \\
\hline c & 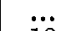 & 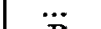 & & $\cdots$ & $\mathbf{M}$ & 1 & & 6438.680 & \\
\hline $\mathrm{Ca} \ldots$ & 10 & $\mathbf{R}$ & 7 & $\ldots$ & $\bigodot^{\prime \prime}$ & $\cdots$ & 11 & $\therefore . .$. & 64 \\
\hline $\mathrm{Ca}$ & 5 & $\cdots$ & 6 & $\cdots$ & $\odot^{\prime \prime}$ & $\cdots$ & 6 & $\because \cdots \cdots$ & 6150 \\
\hline
\end{tabular}

* This is a difficult double or there is a side line close to violet.

+ Chief line in the a group. It is a very close atmospheric double with some weak atmospherio lines towards the red and a faint water-vapour line near to violet side. (J.)

First line of the first pair of lines in the tail of the $a$ group.

Second line in the second pair of the tail of $a$. Faint line to violet.

There is a side line towards the red.

- There is a faint line near to red side.

* There is a Ni line towards red side. 


\begin{tabular}{|c|c|c|c|c|c|c|c|c|c|}
\hline \multirow{2}{*}{ Elements. } & \multicolumn{2}{|c|}{ Is Ano. } & \multicolumn{2}{|c|}{ In Sus. } & \multirow{2}{*}{$\begin{array}{l}\text { Kind } \\
\text { of } \\
\text { Stand } \\
\text { ard. }\end{array}$} & \multicolumn{2}{|c|}{ Weight. } & \multirow{2}{*}{$\begin{array}{l}\text { Ware- } \\
\text { length } \\
\text { in Are. }\end{array}$} & \multirow{2}{*}{\begin{tabular}{|l} 
Wave- \\
length \\
in Sun.
\end{tabular}} \\
\hline & $\begin{array}{c}\text { Inten } \\
\text { sith. }\end{array}$ & $\begin{array}{l}\text { Appear } \\
\text { ance. }\end{array}$ & $\begin{array}{l}\text { Inten- } \\
\text { sity. }\end{array}$ & $\begin{array}{l}\text { Appear- } \\
\text { ance. }\end{array}$ & & $\begin{array}{l}\text { In } \\
\text { Arc. }\end{array}$ & $\begin{array}{c}\text { In } \\
\text { Sun. }\end{array}$ & & \\
\hline $\mathrm{Ca}$ & 10 & $\mathbf{R}$ & 9 & & & & & & \\
\hline $\mathrm{Fe}$ & $?$ & $\ldots$ & 3 & d & $\odot^{\prime \prime}$ & $\ldots$ & 9 & ....... & $6462 \cdot 83$ \\
\hline $\mathrm{Ca}$ & $\mathfrak{5}$ & $\ldots$ & 5 & $\ldots$ & $\odot^{\prime \prime}$ & $\ldots$ & 7 & ....... & 647 \\
\hline$A(w \nabla) \cdot$. & $\ldots$ & $\cdots$ & 1 & $\cdots$ & $\odot$ & $\ldots$ & 6 & ...... & \\
\hline$\stackrel{?}{?} \quad \cdots \cdots$ & 0 & $\cdots$ & 4 & $\cdots$ & $\odot^{\prime \prime}$ & $\ldots$ & 8 & ...... & 64 \\
\hline $\begin{array}{cc}\mathrm{Ca} & \ldots . . \\
\mathrm{Fe} & \ldots .\end{array}$ & 8 & $\cdots$ & 6 & $\cdots$ & $\bigodot^{\prime \prime}$ & $\cdots$ & 10 & ...... & \\
\hline $\begin{array}{ll}\mathrm{Fe} & \ldots . . \\
\mathrm{Ca} & \ldots . .\end{array}$ & $\ddot{5}$ & $\cdots$ & 5 & $\cdots$ & $\bigodot_{\odot}^{\prime \prime}$ & $\cdots$ & $\begin{array}{r}9 \\
10\end{array}$ & …. & $\begin{array}{l}6495 \% 2 \\
6499 \cdot 8\end{array}$ \\
\hline q $\quad \ldots . .$. & 6 & $\cdots$ & $\begin{array}{l}5 \\
4\end{array}$ & ... & $\odot$ & $\ldots$ & 7 & …..... & \\
\hline $\mathrm{Fe}$. & $\cdots$ & $\ldots$ & 4 & $\ldots$ & $\odot^{\prime}$ & $\ldots$ & 10 & ....... & 65 \\
\hline$A(\mathbf{w} \mathbf{v})$ & 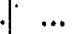 & $\cdots$ & 1 & $\ldots$ & $\odot$ & $\ldots$ & 7 & ....... & 653 \\
\hline$?$ & 19 & $\ldots$ & 3 & $\ldots$ & $\bigodot^{\prime \prime}$ & $\ldots$ & 12 & ........ & $6534 \cdot 17$ \\
\hline $\mathrm{Ti}$ & $3)$ & $\cdots$ & 6 & $\cdots$ & $\odot^{\prime \prime}$ & $\cdots$ & 11 & ( & $6546 \cdot 48$ \\
\hline$A(\mathbf{w v}) \ldots$ & $?$ & $\cdots$ & 2 & & $\odot$ & 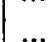 & 6 & $\ldots . .$. & $2 \cdot 84$ \\
\hline CH........... & $\cdots$ & $\cdots$ & 30 & & $\odot^{\prime \prime}$ & … & 13 & …..... & 3.05 \\
\hline Fo $\ldots .$. & $\ldots$ & $\cdots$ & 6 & $\ldots$ & $\bigodot^{\prime \prime}$ & $\ldots$ & 13 & …. & b0 \\
\hline$A(w v) \ldots$ & $\ldots$ & $\ldots$ & 1 & $\ldots$ & $\odot$ & $\ldots$ & 6 & ....... & 65 \\
\hline$?$ & $\ldots$ & $\ldots$ & 2 & $\ldots$ & $\odot$ & ... & 5 & ....... & 65 \\
\hline$F_{\theta}^{*} \ldots \ldots$ & $\ldots$ & $\ldots$ & 4 & $\ldots$ & $\bigodot_{1}$ & $\ldots$ & 7 & ....... & 65 \\
\hline $\mathrm{Fe}$ & $\cdot$ & . & 5 & $\ldots$ & $\bigodot^{\prime \prime}$ & $\ldots$ & 11 & ........ & \\
\hline $\mathrm{Fe} \quad \ldots .$. & $\ldots$ & . & 4 & $\ldots$ & $\bigodot^{\prime \prime}$ & $\ldots$ & 12 & $\ldots .$. & 10 \\
\hline $\mathrm{Fe}$ & $\cdots$ & $\cdots$ & 4 & $\cdots$ & $\bigodot^{\prime \prime}$ & $\cdots$ & 9 & ....... & \\
\hline Fet ...... & $\ldots$ & $\cdots$ & 3 & $\cdots$ & $\odot$ & $\ldots$ & 7 & ....... & 66 \\
\hline $\mathrm{Ni} \quad \ldots . .$. & 5 & $\ldots$ & 5 & $\ldots$ & $\bigodot^{\prime \prime}$ & $\ldots$ & 10 & ....... & \\
\hline$\ldots .$. & $\cdots$ & $\cdots$ & $1)$ & $d$ & $\bigodot^{\prime \prime}$ & $\ldots$ & 4 & ....... & 66 \\
\hline $\mathrm{Fe} \quad \ldots .$. & $\ldots$ & $\ldots$ & 4 & d & $\sigma^{\prime \prime}$ & $\ldots$ & 6 & ....... & \\
\hline $\mathrm{Fe} \quad \ldots .$. & 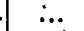 & & 5 & $\cdots$ & $\odot^{\prime \prime}$ & 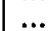 & 10 & ....... & 66 \\
\hline ...... & $\cdots$ & $\ldots$ & 2 & $\ldots$ & $\odot$ & $\ldots$ & 10 & ....... & 670 \\
\hline$? \quad \ldots \ldots$ & 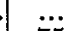 & & 3 & . & $\odot$ & $\cdots$ & 12 & & 670 \\
\hline $\operatorname{Li} \ddagger \ldots . .$. & 75 & t? & $\cdots$ & - & $\mathbf{M}$ & 3 & & $6708 \cdot 07 \mathrm{C}$ & \\
\hline $\mathrm{Ca}$ & 10 & $\ldots$ & 5 & $\cdots$ & $\odot$ & $\ldots$ & 10 & ...... & 67 \\
\hline$? \quad \ldots \ldots$ & $\cdots$ & 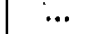 & 3 & $\cdots$ & $\odot$ & $\cdots$ & 10 & ....... & \\
\hline $\mathrm{Fe}$ & $\ldots$ & $\cdots$ & 3 & $\ldots$ & $\odot$ & $\ldots$ & 7 & $\ldots . .$. & 67 \\
\hline$\cdots$ & $\cdots$ & $\ldots$ & 4 & ... & 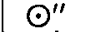 & $\ldots$ & 12 & $\ldots \ldots$ & \\
\hline F & $\ldots$ & & 2 & & $\odot^{\prime}$ & $\ldots$ & 7 & ....... & 67 \\
\hline$\ldots . .$. & 5 & & 4 & $\ldots$ & $\bigodot^{\prime \prime}$ & $\cdots$ & $\dot{9}$ & ....... & \\
\hline ...... & 2 & $\ldots$ & $\mathbf{3}$ & $\ldots$ & $\bigodot^{\prime}$ & $\ldots$ & 10 & ....... & 677 \\
\hline $\mathrm{Fe} \quad \ldots . .$. & $\cdots$ & ... & 1 & ... & ๑ & $\ldots$ & 5 & ....... & 678 \\
\hline ...... & & ... & 2 & . & $\bigodot^{\prime}$ & $\ldots$ & 6 & ........ & \\
\hline $\mathbf{F e}$ & . & $\ldots$ & 3 & $\because$ & $\bigodot^{\prime \prime}$ & $\cdots$ & 8 & ....... & 6810.51 \\
\hline & $\cdot$ & $\cdots$ & 2 & $\ldots$ & $\odot^{\prime}$ & $\ldots$ & 5 & & \\
\hline ..... & $\cdots$ & $\ldots$ & 2 & $\ldots$ & $\odot^{\prime}$ & $\ldots$ & 7 & ........ & 682 \\
\hline $\mathbf{F}$ & & $\cdots$ & 3 & ... & $\bigodot^{\prime}$ & $\cdots$ & 6 & & 6841.50 \\
\hline$\ldots$ & ... & $\ldots$ & 3 & .. & $\bigodot^{\prime}$ & $\ldots$ & 5 & ....... & 68 \\
\hline & 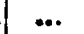 & $\ldots$ & 3 & ... & $\odot^{\prime}$ & $\ldots$ & 10 & ....... & 68 \\
\hline $\mathbf{A}[\mathrm{O}] \|$ & $\cdots$ & .. & 3 & 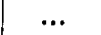 & $\odot^{\prime}$ & $\cdots$ & 11 & ....... & $6867 \cdot 46$ \\
\hline
\end{tabular}

* There is a water-vapour line near to violet side.

$\dagger$ There is a faint line near to each side.

$\ddagger$ With but little material in the are this is a difficult triplet. The violet component is very strong, the red component about half as strong, and between them but nearer the red component is a very narrow line much weaker than either of the others. (J.)

$\S$ There is a side line towards the violet.

il This line and the following one are at the beginning of the head of $B$. There is a fine line midway between them. 


\begin{tabular}{|c|c|c|c|c|c|c|c|c|c|}
\hline \multirow{2}{*}{ Elements. } & \multicolumn{2}{|c|}{ IN ARC. } & \multicolumn{2}{|c|}{ IN Sun. } & \multirow{2}{*}{$\begin{array}{c}\text { Kind } \\
\text { of } \\
\text { Stand- } \\
\text { ard. }\end{array}$} & \multicolumn{2}{|c|}{ WIIGHT. } & \multirow{2}{*}{$\begin{array}{l}\text { Wave- } \\
\text { length } \\
\text { in Arc. }\end{array}$} & \multirow{2}{*}{$\begin{array}{l}\text { Wave- } \\
\text { length } \\
\text { in Sun. }\end{array}$} \\
\hline & $\begin{array}{c}\text { Inten- } \\
\text { sity. }\end{array}$ & $\begin{array}{l}\text { Appear- } \\
\text { ance. }\end{array}$ & $\begin{array}{c}\text { Inten } \\
\text { sity. }\end{array}$ & $\begin{array}{c}\text { Appear- } \\
\text { ance. }\end{array}$ & & $\begin{array}{l}\text { In } \\
\text { Arc. }\end{array}$ & $\begin{array}{c}\text { In } \\
\text { Sun. }\end{array}$ & & \\
\hline $\mathrm{A}[\mathrm{O}]^{*}$. & ... & & 3 & & $\ominus^{\prime}$ & $\ldots$ & 11 & $\ldots . .$. & $6867 \cdot 800$ \\
\hline $\mathbf{A}[\mathrm{O}] \quad \ldots$ & $\ldots$ & $\ldots$ & 1 & "." & $\bigodot_{1}$ & $\ldots$ & 2 & $\ldots \ldots$ & $6868 \cdot 124$ \\
\hline$A[O] \ldots$ & $\ldots$ & $\ldots$ & 6 & d & $\odot$ & $\ldots$ & 2 & ....... & $|6868 \cdot 393|$ \\
\hline $\mathbf{A}(\mathrm{O}) \ldots$ & $\cdots$ & $\cdots$ & $\frac{1}{3}$ & d & $\odot_{1}$ & ... & 3 & ...... & $6868 \cdot 779$ \\
\hline$A(0) i$ & $\cdots$ & ... & 4 & $d$ & $\odot^{\prime}$ & ... & 5 & ...... & $6869 \cdot 141$ \\
\hline$A(0)\}$ & $\ldots$ & $\ldots$ & 4 & & $\odot^{\prime}$ & $\cdots$ & 5 & ....... & $\mid 6869 \cdot 347$ \\
\hline $\mathbf{B A}(\mathrm{O}){ }^{\dagger} \ldots$ & $\cdots$ & $\cdots$ & $\begin{array}{l}4 \\
4\end{array}$ & $d$ & $\odot^{\prime \prime}$ & $\cdots$ & 12 & $\cdots \cdots$ & $6870 \cdot 186$ \\
\hline $\mathbf{A}(\mathrm{O}) \ldots$ & $\ldots$ & ... & $\begin{array}{l}\mathbf{4} \\
5\end{array}$ & $\cdots$ & $\odot^{\prime}$ & ... & 6 & $\ldots \ldots$. & $6871 \cdot 179$ \\
\hline$A(O) \quad \ldots$ & $\ldots$ & $\ldots$ & 5 & ... & $\bigodot^{\prime}$ & $\ldots$ & 6 & ........ & $6871 \cdot 527$ \\
\hline$A(O)$ & $\ldots$ & $\ldots$ & 5 & $\ldots$ & $\odot^{\prime \prime}$ & $\ldots$ & 5 & ....... & $6872 \cdot 493$ \\
\hline$A(O) \quad \ldots$ & $\ldots$ & $\ldots$ & 5 & $\ldots$ & $\odot^{\prime \prime}$ & $\ldots$ & 4 & ....... & $6873 \cdot 076$ \\
\hline$A(0) \quad \ldots$ & $\ldots$ & $\ldots$ & 5 & $\ldots$ & $\odot^{\prime \prime}$ & $\ldots$ & 5 & ........ & $|6874.039|$ \\
\hline$A(O) \quad \ldots$ & $\ldots$ & $\ldots$ & 5 & $\ldots$ & $\bigodot^{\prime \prime}$ & $\ldots$ & 5 & ....... & 6874.884 \\
\hline$A(O) \ldots$ & $\ldots$ & $\ldots$ & 5 & $\ldots$ & $\bigodot^{\prime \prime}$ & $\ldots$ & 9 & ....... & $6875 \cdot 826 \mid$ \\
\hline$A(0) \ldots$ & $\ldots$ & $\ldots$ & 5 & $\ldots$ & $\odot^{\prime \prime}$ & $\ldots$ & 7 & ....... & 6876.957 \\
\hline$A(O) \quad \ldots$ & $\ldots$ & $\ldots$ & 5 & $\ldots$ & $\odot^{\prime \prime}$ & $\ldots$ & 7 & ....... & 6877878 \\
\hline$\vec{A}(\mathrm{O}) \quad \ldots$ & $\ldots$ & $\ldots$ & $\mathbf{5}$ & $\ldots$ & $\odot^{\prime \prime}$ & $\ldots$ & 11 & ....... & 6879.294 \\
\hline$A(O) \ldots$ & & & 3 & $\ldots$ & $\bigodot^{\prime \prime}$ & $\ldots$ & 11 & ........ & $6880 \cdot 176$ \\
\hline $\mathrm{Cr} \ldots \ldots$ & 1 & $\ldots$ & 1 & $\ldots$ & $\odot$ & $\ldots$ & 5 & ....... & 6881.970 \\
\hline Or $\quad \ldots \ldots$ & 2 & $\ldots$ & 1 & $\ldots$ & $\bar{\odot}$ & $\ldots$ & 5 & $\ldots \ldots$ & $6882 \cdot 772$ \\
\hline Or & 3 & $\ldots$ & 1 & $\ldots$ & $\odot$ & $\ldots$ & 5 & $\ldots . .$. & $6883 \cdot 318$ \\
\hline$A(0) \ddagger \ldots$ & $\ldots$ & $\ldots$ & 4 & $\ldots$ & $\odot^{\prime \prime}$ & $\cdots$ & 13 & ....... & 6884.083 \\
\hline $1\{\mathrm{~A}(\mathrm{O})$ & ... & $\ldots$ & 5 & $\ldots$ & $\bigodot^{\prime \prime}$ & $\ldots$ & 12 & ....... & 6886.008 \\
\hline$A(0)$. & $\cdots$ & $\cdots$ & 5 & $\cdots$ & $\bigodot^{\prime \prime}$ & $\ldots$ & 12 & ....... & 68 \\
\hline$A(0)$. & $\ldots$ & $\ldots$ & 5 & $\ldots$ & $\bigodot^{\prime \prime}$ & $\ldots$ & 5 & $\ldots . .$. & $6889 \cdot 194$ \\
\hline $\mathbf{A}(\mathrm{O})$ & $\cdots$ & $\cdots$ & 5 & $\cdots$ & $\odot^{\prime \prime}$ & ... & 5 & ...... & 689 \\
\hline$A(0)$ & $\ldots$ & $\ldots$ & 6 & $\ldots$ & $\odot^{\prime \prime}$ & $\ldots$ & 5 & ....... & $6892 \cdot 614$ \\
\hline$A(0)$ & $\ldots$ & $\ldots$ & 6 & ... & $\bigodot^{\prime \prime}$ & $\ldots$ & 5 & ...... & 68 \\
\hline${ }_{4}\{\mathrm{~A}(\mathrm{O})$. & $\cdots$ & $\cdots$ & 6 & $\cdots$ & $\odot^{\prime \prime}$ & $\cdots$ & 7 & ....... & $6896^{\circ} 29$ \\
\hline${ }^{*}\{A(0)$ & $\cdots$ & $\cdots$ & 6 & $\cdots$ & $\bigodot^{\prime \prime}$ & $\cdots$ & 8 & ...... & $|6897 \cdot 195|$ \\
\hline $5\{A(O)$. & $\cdots$ & $\cdots$ & 6 & $\cdots$ & $\odot_{\rho_{\prime \prime}^{\prime \prime}}^{\prime \prime}$ & $\cdots$ & $\begin{array}{ll}6 \\
8\end{array}$ & ....... & $|6900 \cdot 199|$ \\
\hline$A(0)$ & ... & $\cdots$ & 6 & ... & $\bigodot_{\prime \prime \prime}^{\prime \prime}$ & $\cdots$ & 8 & $\ldots \ldots$ & $6901 \cdot 113$ \\
\hline$A(0)$. & $\cdots$ & $\cdots$ & 6 & $\cdots$ & $\odot_{\prime \prime \prime}^{\prime \prime}$ & $\cdots$ & 5 & ....... & 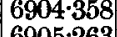 \\
\hline$A(0)$. & $\cdots$ & $\cdots$ & 6 & $\cdots$ & $\bigodot^{\prime \prime}$ & $\cdots$ & 5 & ...... & $|6905.263|$ \\
\hline$A(O)$. & $\cdots$ & . & 6 & $\cdots$ & $\bigodot^{\prime \prime}$ & $\cdots$ & $\begin{array}{l}5 \\
9\end{array}$ & ...... & $\left|\begin{array}{l}6908 \cdot 785 \\
6009 \cdot 675\end{array}\right|$ \\
\hline A(O). & $\cdots$ & $\cdots$ & $\begin{array}{l}0 \\
5\end{array}$ & ... & $\stackrel{\odot}{\odot}^{\prime \prime}$ & ... & 5 & ….... & $\mid \begin{array}{l}0909.675 \\
6913.454\end{array}$ \\
\hline $8\{\vec{A}(\mathrm{O})$. & ... & $\cdots$ & 5 & $\cdots$ & $\odot^{\prime \prime}$ & .... & 4 & ......... & 6914.328 \\
\hline Ni $\quad . . .$. & 5 & $\ldots$ & 3 & $\ldots$ & $\bigodot^{\prime}$ & $\ldots$ & 4 & ....... & $6914 \cdot 819$ \\
\hline ? ... & $\cdots$ & $\ldots$ & 2 & $\ldots$ & $\odot^{\prime}$ & $\cdots$ & 5 & ....... & $6916 \cdot 957$ \\
\hline$g\{A(O)$. & $\ldots$ & $\cdots$ & 4 & $\ldots$ & $\odot_{u}^{\prime \prime}$ & $\ldots$ & 5 & ....... & 6918.363 \\
\hline$\lceil\mathrm{A}(\mathrm{O})$ & ... & $\ldots$ & 4 & $\ldots$ & $\odot^{\prime \prime}$ & $\ldots$ & 9 & ....... & $6919 \cdot 24$ \\
\hline${ }_{10}\{A(O)$. & $\ldots$ & $\ldots$ & 3 & $\ldots$ & $\odot^{\prime \prime}$ & $\ldots$ & 8 & ....... & 6923.557 \\
\hline I $A(0)$ & $\ldots$ & $\ldots$ & 3 & ... & $\bigodot^{\prime \prime}$ & ... & 11 & ...... & $6924 \cdot 420$ \\
\hline $1\{A(0)$. & $\ldots$ & $\cdots$ & 2 & ... & $\bigodot^{\prime}$ & $\ldots$ & 5 & ....... & 6928.992 \\
\hline$A(0)$. & $\cdots$ & $\cdots$ & 2 & $\cdots$ & $\bigodot^{\prime}$ & ... & 8 & ...... & $6929 \cdot 8$ \\
\hline $12\left\{\begin{array}{l}A(O) . \\
A(O) .\end{array}\right.$ & $\begin{array}{l}\cdots \\
\ldots\end{array}$ & $\cdots$ & $\begin{array}{l}1 \\
1\end{array}$ & $\begin{array}{l}\cdots \\
\ldots\end{array}$ & $\stackrel{\odot}{\odot}$ & $\cdots$ & $\begin{array}{l}\mathbf{4} \\
5\end{array}$ & ….... & 6935.530 \\
\hline & & & & & & & & & \\
\hline
\end{tabular}

* This line and the preceding one are at the beginning of the head of $\mathbf{R}$. There is a fine line midway between them. + The principal line in the head of $B$. It is a difficult double $\$$ Single line at the beginning of the tail of $B$. 


\begin{tabular}{|c|c|c|c|c|c|c|c|c|c|}
\hline \multirow{2}{*}{ Elements. } & \multicolumn{2}{|c|}{ IN ARC. } & \multicolumn{2}{|c|}{ IN Sun. } & \multirow{2}{*}{$\begin{array}{l}\text { Kind } \\
\text { of } \\
\text { Stand- } \\
\text { ard. }\end{array}$} & \multicolumn{2}{|c|}{ W HIGHT. } & \multirow{2}{*}{$\begin{array}{l}\text { Wave- } \\
\text { length } \\
\text { in Arc. }\end{array}$} & \multirow{2}{*}{$\begin{array}{l}\text { Wave- } \\
\text { length } \\
\text { in Sun. }\end{array}$} \\
\hline & $\begin{array}{c}\text { Inten- } \\
\text { sity. }\end{array}$ & $\begin{array}{l}\text { Appear- } \\
\text { ance. }\end{array}$ & $\begin{array}{l}\text { Inten } \\
\text { sity. }\end{array}$ & $\begin{array}{c}\text { Appear- } \\
\text { ance. }\end{array}$ & & $\begin{array}{l}\text { In } \\
\text { Are. }\end{array}$ & $\begin{array}{l}\text { In } \\
\text { Sun. }\end{array}$ & & \\
\hline$A(w v ?) \ldots$ & $\ldots$ & $\cdots$ & 8 & $\cdots$ & $\odot^{\prime \prime}$ & $\cdots$ & 10 & $\ldots . . .$. & $6947 \cdot 78$ \\
\hline & 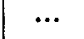 & $\cdots$ & $2\}$ & $d$ & $\bigodot_{1}$ & $\cdots$ & 4 & ....... & 695383 \\
\hline & $\cdots$ & $\cdots$ & 15 & & " & ".” & & (n.... & \\
\hline $\overrightarrow{A C}$ & $\cdots$ & $\cdots$ & $\begin{array}{l}8 \\
3\end{array}$ & $\cdots$ & $\bigodot^{\prime \prime}$ & $\cdots$ & $\begin{array}{l}12 \\
10\end{array}$ & $\cdots \cdots$ & $\begin{array}{l}956 \cdot 700 \\
959 \cdot 708\end{array}$ \\
\hline $\begin{array}{l}A(r) \\
A(r)\end{array}$ & 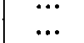 & ... & $\begin{array}{l}3 \\
6\end{array}$ & $\cdots$ & $\stackrel{\odot}{\odot}$ & $\begin{array}{l}\cdots \\
\cdots\end{array}$ & $\begin{array}{l}10 \\
12\end{array}$ & ….. & 6961.51 \\
\hline & 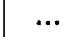 & $\ldots$ & 2 & $\ldots$ & 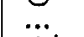 & & 5 & ........ & $6978 \cdot 65$ \\
\hline$A(\pi$ & $\cdots$ & $\cdots$ & 5 & $\ldots$ & $\odot^{\prime}$ & $\ldots$ & 10 & ........ & 6986.8 \\
\hline$A(x$ & $\ldots$ & $\cdots$ & 5 & ․ㅏ & $\odot$ & $\ldots$ & 7 & $\ldots . .$. & $6989 \cdot 24$ \\
\hline P?) $\dagger$ & $\cdots$ & $\cdots$ & 5 & $\mathrm{~d} ?$ & $\bigodot_{1}$ & $\cdots$ & 6 & $\ldots \ldots$ & $6999 \cdot 17$ \\
\hline ........ & $\cdots$ & $\cdots$ & 4 & $\cdots$ & $\odot$ & $\cdots$ & 3 & ....... & $7000 \cdot 1$ \\
\hline & $\cdots$ & $\cdots$ & 5 & ... & $\bigodot_{1}$ & $\cdots$ & 5 & ...... & $7006 \cdot 1$ \\
\hline$? \ldots \ldots \ldots$ & $\cdots$ & $\cdots$ & 3 & $\cdots$ & $\odot$ & $\cdots$ & 6 & ...... & $7011 \%$ \\
\hline$A(w v ?) \ldots$ & $\cdots$ & $\cdots$ & $3\}$ & $\cdots$ & $\bigodot_{1}$ & $\cdots$ & 5 & ....... & 7016 \\
\hline$A(w \vee ?) \ldots$ & $\cdots$ & $\cdots$ & 6 & $\cdots$ & $\bigodot_{1}$ & $\ldots$ & 9 & $\ldots \ldots$ & $7016 \cdot 6$ \\
\hline & 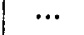 & $\cdots$ & 4 & $\cdots$ & $\bigodot_{1}$ & $\cdots$ & 1 & ....... & $7023 \cdot 22$ \\
\hline ........ & $\cdots$ & $\cdots$ & 3 & & $\bigodot_{1}$ & $\cdots$ & 7 & $\ldots .$. & $7023 \cdot 74$ \\
\hline ....... & $\cdots$ & $\ldots$ & 3 & $d ?$ & $\odot_{1}$ & $\cdots$ & 2 & ....... & 7024.98 \\
\hline ? ............ & $\cdots$ & $\cdots$ & $1\}$ & $\cdots$ & $\cdots$ & $\cdots$ & 1 & ...... & 702 \\
\hline$?$ & $\cdots$ & $\cdots$ & $3\}$ & $\ldots$ & & $\ldots$ & 7 & ....... & 702 \\
\hline$? \ldots$ & $\cdots$ & $\cdots$ & 6 & $\cdots$ & $\odot^{\prime}$ & $\cdots$ & 8 & ...... & 70 \\
\hline$? \ldots$ & $\cdots$ & ... & 2 & $\cdots$ & & $\cdots$ & 6 & ....... & 703 \\
\hline$? \ldots$ & 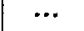 & $\ldots$ & 4 & $\cdots$ & $\odot$ & $\cdots$ & 10 & ....... & 704 \\
\hline$? \ldots$ & $\cdots$ & .. & 4 & $\cdots$ & $\bigodot^{\prime}$ & $\cdots$ & 5 & ....... & 709 \\
\hline$?$ & .. & $\cdots$ & 6 & $\cdots$ & $\odot^{\prime}$ & $\cdots$ & 5 & ....... & 718 \\
\hline$? \ldots$ & 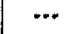 & $\cdots$ & 1 & $\cdots$ & $\dddot{m}$ & $\cdots$ & 4 & $\cdots \ldots$ & 714 \\
\hline & 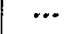 & $\cdots$ & 7 & $\cdots$ & $\odot^{\prime}$ & $\cdots$ & 4 & ...... & \\
\hline$?$ & - & $\ldots$ & 3 & .* & ... & $\cdots$ & 5 & ...... & 71 \\
\hline$A(w$ & .. & & 3 & 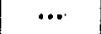 & $\cdots$ & . & 6 & $\ldots \ldots$ & \\
\hline$A(w \nabla ?) \ldots$ & & & 4 & & $\ldots$ & $\theta$ & 7 & $\ldots \ldots$ & 718 \\
\hline$A(w v ?) \ldots$ & 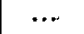 & 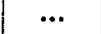 & 5 & d & $\ldots$ & $\cdots$ & 6 & ....... & \\
\hline$A(w v) \ldots$ & & . & 7 & $\cdots$ & & $\ldots$ & 3 & $\cdots$ & 719 \\
\hline$A(\mathbf{w}) \ldots$ & .. & $\cdots$ & 10 & 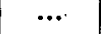 & $\odot$ & $\ldots$ & 5 & .... & \\
\hline$A(w v) \ldots$ & 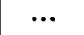 & & 10 & & $\odot$ & $\cdots$ & 5 & ....... & 720 \\
\hline$A($ (พv?)... & ... & $\ldots$ & 6 & $\ldots$ & & $\cdots$ & 4 & ....... & \\
\hline & $\cdots$ & - & 8 & & $\odot$ & $\cdots$ & 5 & ....... & 7223.93 \\
\hline$? \ldots$. & $\cdots$ & $\cdots$ & 6 & $\cdots$ & $\cdots$ & $\cdots$ & 4 & ....... & 7227 \\
\hline$? .$. & 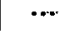 & ... & 3 & & & $\cdots$ & 3 & ...... & \\
\hline & 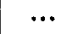 & ... & 8 & $\cdots$ & $\odot$ & $\cdots$ & 4 & ....... & 726 \\
\hline$\Delta(\mathrm{wv}) \ldots$ & 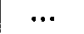 & 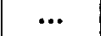 & 4 & & $\dddot{\dddot{\prime}}$ & $\cdots$ & 5 & ....... & 7240.97 \\
\hline$A(w r) \ldots$ & .. & 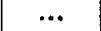 & 15 & ... & $\odot$ & 1 & 4 & ....... & 724 \\
\hline$A(w v) ? \ldots$ & .. & . & 4 & $\ldots$ & $\cdots$ & $\ldots$ & 2 & ....... & 724 \\
\hline$A(\mathbf{w}) ? \ldots$ & .. & & 8 & & 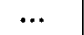 & .. & 3 & ....... & 7264.85 \\
\hline$A(w v) ? \ldots$ & .. & ... & 8 & $\ldots$ & $\ldots$ & $\ldots$ & 3 & ....... & $7265-83$ \\
\hline & & & 3 & d & $\cdots$ & 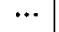 & 2 & ....... & $7270 \cdot 20$ \\
\hline$A(\mathrm{wv}) ? .$. & $\ldots$ & . & 8 & $\ldots$ & $\ldots$ & $\cdots$ & 4 & ....... & 7273 \\
\hline $\mathbf{A}(\mathbf{w}) ? \cdots$ & ... & 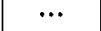 & 6 & & • & $\cdots$ & 3 & ...... & $7287 \cdot 68$ \\
\hline$A(w v) ? \ldots$ & $\ldots$ & .. & 10 & $\mathrm{~d} ?$ & 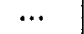 & $\cdots$ & 3 & ....... & $7290 \cdot 71$ \\
\hline$A(w v) \ldots$ & $\cdots$ & $\ldots$ & 4 & $\cdots$ & $\cdots$ & $\cdots$ & 3 & $\ldots \ldots$ & 730005 \\
\hline$A(w \boldsymbol{v}) ?$. & $\ldots$ & $\cdots$ & 7 & & ... & 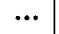 & 4 & $\ldots . .$. & $7304 \cdot 47$ \\
\hline$A(w v) ? \ldots$ & $\cdots$ & $\cdots$ & 5 & d? & $\cdots$ & $\cdots$ & 4 & $\ldots \ldots$ & $7318 \cdot 81$ \\
\hline & $\cdots$ & $\cdots$ & 2 & $\cdots$ & $\cdots$ & $\cdots$ & 3 & ...... & 7321.056 \\
\hline
\end{tabular}

* There is a line towards the violet.

+ There is a line close to violet side. 
Drawing of Curves by their Curvature.

\begin{tabular}{|c|c|c|c|c|c|c|c|c|c|}
\hline \multirow{2}{*}{ Elements. } & \multicolumn{2}{|c|}{ IN ARC. } & \multicolumn{2}{|c|}{ IN Sun. } & \multirow{2}{*}{$\begin{array}{l}\text { Kind } \\
\text { of } \\
\text { Stand- } \\
\text { ard. }\end{array}$} & \multicolumn{2}{|c|}{ WEIGHT. } & \multirow{2}{*}{$\begin{array}{l}\text { Wave- } \\
\text { length } \\
\text { in Arc. }\end{array}$} & \multirow{2}{*}{$\begin{array}{l}\text { Wave- } \\
\text { length } \\
\text { in Sun. }\end{array}$} \\
\hline & $\begin{array}{l}\text { Inten- } \\
\text { sity. }\end{array}$ & $\begin{array}{c}\text { Appear } \\
\text { ance. }\end{array}$ & $\begin{array}{l}\text { Inten- } \\
\text { sity. }\end{array}$ & & & $\begin{array}{l}\text { In } \\
\text { Aro. }\end{array}$ & In & & \\
\hline 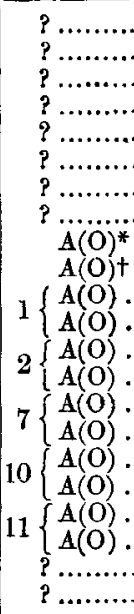 & 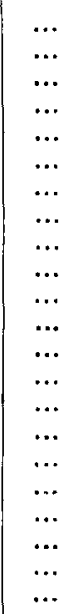 & $\begin{array}{l}\cdots \\
\cdots \\
\cdots \\
\cdots \\
\cdots \\
\cdots \\
\cdots \\
\cdots \\
\cdots \\
\cdots \\
\cdots \\
\cdots\end{array}$ & $\begin{array}{r}10 \\
12 \\
12 \\
14 \\
14 \\
14 \\
14 \\
8 \\
8 \\
7 \\
7 \\
7 \\
4\end{array}$ & 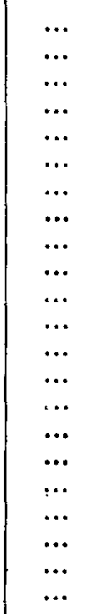 & $\begin{array}{l}\ldots \\
\ldots \\
\ldots \\
\ldots \\
\ldots \\
\ldots \\
\ldots \\
\ldots \\
\ldots \\
\bigodot^{\prime} \\
\odot \\
\odot \\
\odot \\
\odot \\
\odot \\
\odot \\
\ldots \\
\ldots \\
\ldots \\
\ldots \\
\ldots \\
\ldots\end{array}$ & $\begin{array}{l}\ldots . \\
\ldots \\
\ldots \\
\ldots \\
\ldots \\
\ldots . \\
\ldots \\
\ldots \\
\ldots \\
\ldots \\
\ldots \\
\ldots \\
\ldots \\
\ldots \\
\ldots \\
\ldots \\
\ldots \\
\ldots\end{array}$ & $\begin{array}{l}3 \\
2 \\
2 \\
3 \\
2 \\
3 \\
3 \\
1 \\
1 \\
4 \\
5 \\
4 \\
4 \\
3 \\
3 \\
3 \\
3 \\
3 \\
3 \\
3 \\
3 \\
3 \\
1 \\
1\end{array}$ & 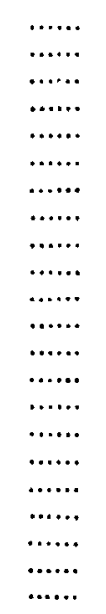 & \begin{tabular}{|c|}
$7594 \cdot 059$ \\
$7621 \cdot 277$ \\
$7623 \cdot 526$ \\
$7624 \cdot 853$ \\
$7627 \cdot 232$ \\
$7628 \cdot 585$ \\
$7659 \cdot 658$ \\
$7660 \cdot 778$ \\
$7665 \cdot 265$ \\
$7666 \cdot 239$ \\
$7670 \cdot 993$ \\
$7671 \cdot 994$ \\
$7699 \cdot 274$ \\
$7714 \cdot 686$
\end{tabular} \\
\hline
\end{tabular}

* Beginning of the head of $A$. Outside edge.

+ Single line at the beginning of the tail of $A$.

\section{On the Drawing of Curves by their Curvature. By C. V. Boys, A.R.S.M., F.R.S.*}

\section{[Plate I.]}

\section{$\mathrm{W}$}

HILE giving a course of lectures to working men on Capillarity at the R. School of Mines in 1891, I wished to explain the principles upon which the form of a water-drop depends, and to show that an accurate scaledrawing could aetually be produced by following Lord Kelvin's rule (Proc. R. Inst. Jan. 29, 1886) ; but found that the procedure was so cumbersome as to be in no way adapted to popular exposition. In my attempt to simplify the operation I devised a method of carrying it out which has the double advantage that any of these capillary surfaces of revolution can be drawn with a facility which cannot be approached by following Lord Kelvin's instructions explicitly, while the accuracy of any curve thus determined is so great

* Communicated by the Physical Society : read May 12, 1893. 